\title{
SARS-CoV-2 and the role of close contact in transmission: a
}

\section{systematic review [version 1; peer review: 1 approved with}

\section{reservations, 1 not approved]}

\author{
Igho J. Onakpoya (D1), Carl J. Heneghan (D1), Elizabeth A. Spencer (D1), \\ Jon Brassey (iD2, Annette Plüddemann1, David H. Evans (iD), John M. Conly4, \\ Tom Jefferson ${ }^{1}$
}

\footnotetext{
${ }^{1}$ Nuffield Department of Primary Care Health Sciences, University of Oxford, Oxford, OX2 6GG, UK

${ }^{2}$ Trip Database Ltd, Newport, NP20 3PS, UK

${ }^{3}$ Department of Medical Microbiology \& Immunology,Li Ka Shing Institute of Virology, University of Alberta, Edmonton, AB, T6G 2R3, Canada

4University of Calgary and Alberta Health Services,, University of Calgary, Calgary, AB, T2N 4Z6, Canada
}

V1 First published: 09 Apr 2021, 10:280

https://doi.org/10.12688/f1000research.52439.1

Second version: $06 \mathrm{Jul} 2022,10: 280$

https://doi.org/10.12688/f1000research.52439.2

Latest published: 17 Nov 2022, 10:280

https://doi.org/10.12688/f1000research.52439.3

\section{Abstract}

Background: SARS-CoV-2 transmission has been reported to be associated with close contact with infected individuals. However, the mechanistic pathway for transmission in close contact settings is unclear. Our objective was to identify, appraise and summarise the evidence from studies assessing the role of close contact in SARS-CoV2 transmission.

Methods: This review is part of an Open Evidence Review on Transmission Dynamics of SARS-CoV-2. We conduct ongoing searches using WHO Covid-19 Database, LitCovid, medRxiv, PubMed and Google Scholar; assess study quality based on the QUADAS-2 criteria and report important findings on an ongoing basis.

Results: We included 181 studies: 171 primary studies and 10 systematic reviews. The settings for primary studies were predominantly in home/quarantine facilities (31.6\%) and acute care hospitals (15.2\%). The overall reporting quality of the studies was low to moderate. There was significant heterogeneity in design and methodology. The frequency of attack rates (PCR testing) was 3.5-75\%; attack rates were highest in prison and wedding venues, and in households. The frequency of secondary attack rates was 0.3-100\% with rates highest in home/quarantine settings. Three studies showed no transmission if index cases had recurrent infection. Viral culture was performed in three studies of which two found viable virus; culture results were negative where index cases had recurrent

Open Peer Review
Approval Status X ?
version 3
(revision)
17 Nov 2022
version 2
(revision)
06 Jul 2022
version 1
09 Apr 2021
……………….........................................................
1. Kevin Escandón (iD), University of Minnesota
Medical School, Minneapolis, USA
Angela K. Ulrich, University of Minnesota,
Minneapolis, USA
University of Minnesota, Minneapolis, USA
2. Richard Wamai, Northeastern University,
Japan


infections. Ten studies performed genomic sequencing with phylogenetic analysis - the completeness of genomic similarity ranged from $81-100 \%$. Findings from systematic reviews showed that children were significantly less likely to transmit SARS-CoV-2 and household contact was associated with a significantly increased risk of infection.

Conclusions: The evidence from published studies demonstrates that SARS-CoV-2 can be transmitted via close contact settings. The risk of transmission is greater in household contacts. There was wide variation in methodology. Standardized guidelines for reporting transmission in close contact settings should be developed to improve the quality reporting.

\section{Keywords}

Close contact, transmission, COVID-19, systematic review

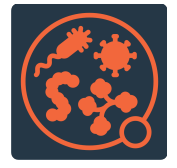

This article is included in the Pathogens

gateway.

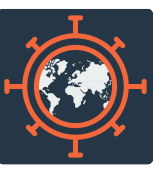

This article is included in the Emerging Diseases and Outbreaks gateway.

\section{8.}

This article is included in the Coronavirus

collection.
4. Gary Lin (iD), One Health Trust, Silver Spring,

USA

Johns Hopkins University, Baltimore, USA

Any reports and responses or comments on the article can be found at the end of the article. 


\section{Corresponding author: Igho J. Onakpoya (igho.onakpoya@conted.ox.ac.uk)}

Author roles: Onakpoya IJ: Conceptualization, Data Curation, Formal Analysis, Methodology, Writing - Original Draft Preparation, Writing - Review \& Editing; Heneghan CJ: Conceptualization, Data Curation, Formal Analysis, Funding Acquisition, Investigation, Methodology, Supervision, Writing - Original Draft Preparation, Writing - Review \& Editing; Spencer EA: Data Curation, Formal Analysis, Methodology, Writing - Original Draft Preparation, Writing - Review \& Editing; Brassey J: Methodology, Resources, Writing - Original Draft Preparation, Writing - Review \& Editing; Plüddemann A: Writing - Original Draft Preparation, Writing - Review \& Editing; Evans DH : Formal Analysis, Methodology, Writing - Original Draft Preparation, Writing - Review \& Editing; Conly JM: Conceptualization, Data Curation, Writing - Original Draft Preparation, Writing - Review \& Editing; Jefferson T: Conceptualization, Data Curation, Formal Analysis, Funding Acquisition, Methodology, Writing - Original Draft Preparation, Writing - Review \& Editing

Competing interests: $\mathrm{CJH}$ holds grant funding from the NIHR School of Primary Care Research, the NIHR BRC Oxford and the World Health Organization for a series of Living rapid review on the modes of transmission of SARs-CoV-2 reference WHO registration No2020/1077093. He has received expenses and fees for his media work. He receives expenses for teaching EBM and is also paid for his GP work in NHS out of hours (contract Oxford Health NHS Foundation Trust) and for appraising treatment recommendations in non-NHS settings. He is the Director of CEBM and is an NIHR Senior Investigator. TJ was in receipt of a Cochrane Methods Innovations Fund grant to develop guidance on the use of regulatory data in Cochrane reviews (2015-018). In 2014-2016, he was a member of three advisory boards for Boehringer Ingelheim. TJ was a member of an independent data monitoring committee for a Sanofi Pasteur clinical trial on an influenza vaccine. TJ is occasionally interviewed by market research companies about phase I or II pharmaceutical products for which he receives fees (current). TJ was a member of three advisory boards for Boehringer Ingelheim (2014-16). TJ was a member of an independent data monitoring committee for a Sanofi Pasteur clinical trial on an influenza vaccine (2015-2017). TJ is a relator in a False Claims Act lawsuit on behalf of the United States that involves sales of Tamiflu for pandemic stockpiling. If resolved in the United States' favor, he would be entitled to a percentage of the recovery. TJ is co-holder of a Laura and John Arnold Foundation grant for development of a RIAT support centre (2017-2020) and Jean Monnet Network Grant, 2017-2020 for The Jean Monnet Health Law and Policy Network. TJ is an unpaid collaborator to the project Beyond Transparency in Pharmaceutical Research and Regulation led by Dalhousie University and funded by the Canadian Institutes of Health Research (2018-2022). TJ consulted for Illumina LLC on next generation gene sequencing (2019-2020). TJ was the consultant scientific coordinator for the HTA Medical Technology programme of the Agenzia per $i$ Serivizi Sanitari Nazionali (AGENAS) of the Italian MoH (2007-2019). TJ is Director Medical Affairs for BC Solutions, a market access company for medical devices in Europe. TJ is funded by NIHR UK and the World Health Organization (WHO) to update Cochrane review A122, "Physical Interventions to interrupt the spread of respiratory viruses". TJ is funded by Oxford University to carry out a living review on the transmission epidemiology of COVID-19. Since 2020, TJ receives fees for articles published by The Spectator and other media outlets. TJ is part of a review group carrying out "Living rapid literature review on the modes of transmission of SARS-CoV-2 (WHO Registration 2020/1077093-0)". He is a member of the WHO COVID-19 Infection Prevention and Control Research Working Group. DHE has been awarded U.S. patents as a co-inventor of related oncolytic virus technologies and is a co-owner of Prophysis Inc., which retains a partial interest in the licensing rights for these technologies. JMC holds grants from the Canadian Institutes for Health Research on acute and primary care preparedness for COVID-19 in Alberta, Canada and was the primary local Investigator for a Staphylococcus aureus vaccine study funded by Pfizer for which all funding was provided only to the University of Calgary. He also received support from the Centers for Disease Control and Prevention (CDC) to attend an Infection Control Think Tank Meeting. Annette Plüddemann is Senior Research Fellow at the Centre for Evidence-Based Medicine and reports grant funding from NIHR School of Primary Care Research (NIHR SPCR ESWG project 390 and project 461), during the conduct of the study, and occasionally receives expenses for teaching Evidence-Based Medicine. IJO, EAS and JB have no interests to disclose.

Grant information: The review was funded by the World Health Organization: Living rapid review on the modes of transmission of SARs-CoV-2 reference WHO registration No2020/1077093. CH and ES also receive funding support from the NIHR SPCR Evidence Synthesis Working Group project 390.

The funders had no role in study design, data collection and analysis, decision to publish, or preparation of the manuscript.

Copyright: @ 2021 Onakpoya IJ et al. This is an open access article distributed under the terms of the Creative Commons Attribution License, which permits unrestricted use, distribution, and reproduction in any medium, provided the original work is properly cited.

How to cite this article: Onakpoya IJ, Heneghan CJ, Spencer EA et al. SARS-CoV-2 and the role of close contact in transmission: a systematic review [version 1; peer review: 1 approved with reservations, 1 not approved] F1000Research 2021, 10:280 https://doi.org/10.12688/f1000research.52439.1

First published: 09 Apr 2021, 10:280 https://doi.org/10.12688/f1000research.52439.1 


\section{Introduction}

The SARS-CoV-2 (COVID-19) pandemic is a major public health concern. Based on WHO data, there have been over 120 million confirmed cases and over two and a half million deaths globally as of 20th March $2021^{1}$. Many national governments have implemented prevention and control measures and vaccines are now being approved and administered; the overall global spread of the virus now appears to be slowing. Current evidence from epidemiologic and virologic studies suggest SARS-CoV-2 is primarily transmitted via respiratory droplets and direct and indirect contact ${ }^{2,3}$. However, controversy still exists about how the virus is transmitted and the relative frequency of the modes of transmission and if these modes may be altered in specific settings $\mathrm{s}^{4,5}$.

Although close contact is thought to be associated with transmission of SARS-CoV-2, there is uncertainty about the thresholds of proximity for "close contact" and the factors that may influence the transmission in a "close contact". Furthermore, there is lack of clarity about how research should be conducted in the setting of transmission with close contact which may include transmission via any one of or the combination of respiratory droplets, direct contact, or indirect contact.

Several studies investigating the role of close contact in SARS-CoV-2 transmission have been published but the pathways and thresholds for transmission are not well established. The objective of this review was to identify, appraise and summarize the evidence from primary studies and systematic reviews investigating the role of close contact in the transmission of SARS-CoV-2. Terminology for this article can be found in Box 1.

\section{Box 1. Terminology}

Close contact: Someone who was within 6 feet of an infected person for a cumulative total of 15 minutes or more over a 24-hour period starting from 2 days before illness onset (or, for asymptomatic patients, 2 days prior to test specimen collection) until the time the patient is isolated; ${ }^{1}$ The World Health Organization (WHO) additionally includes direct physical contact with a probable or confirmed case, direct care for a patient with probable or confirmed COVID-19 disease without using proper PPE, and other situations as indicated by local risk assessments.

Attack rate: The proportion of those who become ill after a specified exposure

Secondary attack rate: The probability that infection occurs among susceptible persons within a reasonable incubation period following known contact with an infectious person or an infectious source ${ }^{3}$.

Cycle threshold: The number of cycles required for the fluorescent signal to cross the threshold. Ct levels are inversely proportional to the amount of target nucleic acid in the sample ${ }^{4}$.

1https://www.cdc.gov/coronavirus/2019-ncov/global-covid-19/ operational-considerations-contact-tracing.html\#: :text=Close contact is defined by, time the patient is isolated

2https://www.who.int/foodsafety/publications/foodborne_disease/ Annex_7.pdf

${ }^{3}$ Halloran ME. Secondary Attack Rate. In: Peter A, Theodore C, editors. Encyclopedia of Biostatistics. New York: John Wiley \& Sons Ltd; 2005

4https://www.ncbi.nlm.nih.gov/pmc/articles/PMC7521909/

\section{Methods}

We are undertaking an open evidence review examining the factors and circumstances that impact on the transmission of SARS-CoV-2, based on our published protocol last updated on the 1 December 2020 (Version 3: 1 December 2020, Extended data: Appendix $1^{6}$ ). This review aims to identify, appraise, and summarize the evidence (from peer-reviewed studies or studies awaiting peer review) examining the role of close contact in the transmission of SARS-CoV-2 and the factors that influence transmissibility. We are conducting an ongoing search in WHO Covid-19 Database, LitCovid, medRxiv, and Google Scholar for SARS-CoV-2 for keywords and associated synonyms. For this review, we also conducted searches on PubMed. The searches for this update were conducted up to 20th December 2020 (Extended data: Appendix $2^{6}$ ). We did not impose any language restrictions.

We included studies of any design that investigated transmission associated with close contact but excluded predictive or modelling studies. We reviewed the results for relevance and for articles that appeared particularly relevant, we undertook forward citation matching to identify relevant results. We assessed the risk of bias of included primary studies using five domains from the QUADAS-2 criteria; ${ }^{7}$ we adapted this tool because the included studies were not primarily designed as diagnostic accuracy studies. We did not perform formal assessments of the quality of included systematic reviews but summarized their findings, including quality of their included studies as reported by the authors. We extracted the following information from included studies: study design characteristics including the definition used of "close contact", population, main methods, and associated outcomes including the number of swab samples taken with frequency and timing of samples, and cycle thresholds and samples concentrations. We also extracted information on viral cultures including the methods used. One reviewer (IJO) assessed the risk of bias from primary studies, and these were independently verified by a second reviewer (EAS). One reviewer (IJO) extracted data from the included primary studies, and these were independently checked by a second reviewer $(\mathrm{CJH})$. One reviewer $(\mathrm{CJH})$ extracted data from the included systematic reviews, and these were independently checked by a second reviewer (IJO). Disagreements in the data extraction or bias assessments were resolved by consensus. We presented the results in tabular format, and bar charts used to present the frequency of positive tests. We reported results of specific subgroups of studies where relevant. Because of substantial heterogeneity across the included studies, we considered meta-analyses inappropriate.

\section{Results}

We identified 1202 non-duplicate citations of which 229 were considered eligible (Figure 1). We excluded 48 full-text studies for various reasons (see Extended data: Appendix $3^{6}$ for the list of excluded studies and reasons for exclusion). Finally, we included 181 studies: 171 primary studies and 10 systematic reviews (see Extended data: Appendix 4 for references to included studies). The main characteristics of the included primary studies and systematic reviews are shown in Table 1 and Table 2, respectively. 


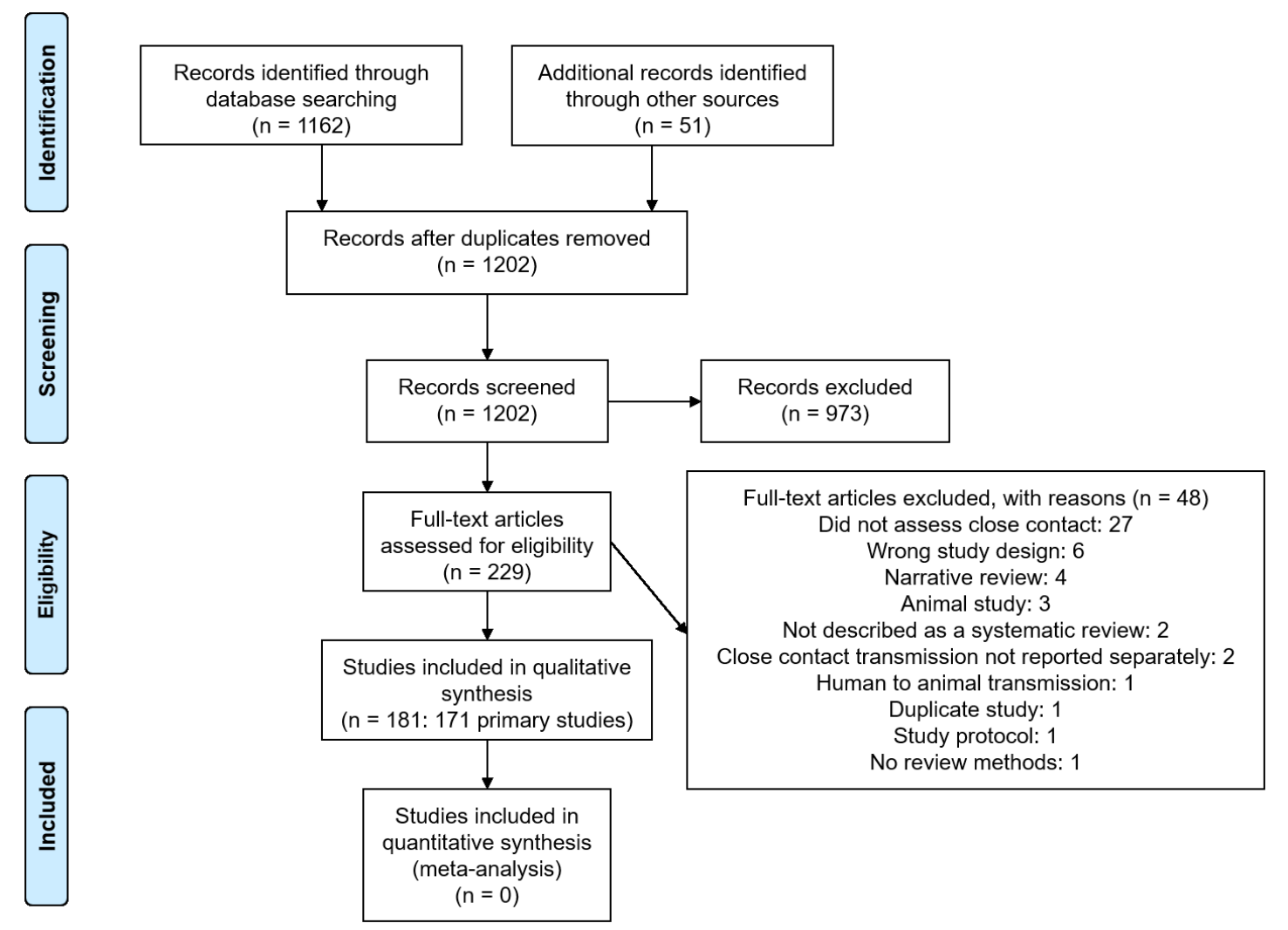

Figure 1. Flow diagram showing the process for inclusion of studies assessing close contact transmission in SARS-CoV-2.

\section{Quality of included studies}

None of the included primary studies reported a published protocol except one (Helsingen 2020). The risk of bias of the included primary studies is shown in Table 5. Only 61 studies (35.7\%) adequately reported the methods used, and 97 (56.7\%) adequately described the sources of sample collection. Only six studies $(3.5 \%)$ adequately reported methods used to address biases. The overall quality of the studies was judged as low to moderate (see the risk of bias graph in Figure 2).

\section{Reviews}

We included 10 systematic reviews investigating the role of close contact in SARS-CoV-2 transmission (Table 2). The studies included in the reviews were primarily observational. In one review (Chen 2020), there was a higher risk of infection in close contacts and healthcare workers without PPE compared to the general population. A second review (Chu 2020) found a significant association between proximity of exposure (distance $<1 \mathrm{~m}$ ), absence of barriers (not using face covering or eye protection) and the risk of infection. The authors of three reviews (Li 2020, Ludvigsson 2020, Zhu 2020) concluded that children were unlikely to be the main conduit for transmission of SARS-CoV-2, and results of one review (Koh 2020) showed that adults with close contact exposure were significantly more likely to be infected compared with children (14 studies, RR:
1.71 (95\% CI: 1.35, 2.17)). In one review (Xu 2020), the attack rates were significantly less in students compared with staff $(\mathrm{p}<0.01)$. One review (Fung 2020) reported household SARs ranging from $3.9 \%$ to $36.4 \%$, but also highlighted the lack of SARS-CoV-2 research in Africa, South Asia, and Latin America. One review (Madewell 2020) found that SARs were higher in households from symptomatic index cases than asymptomatic index cases, and one review (Yanes-Lane 2020) concluded that the proportion of asymptomatic infection was high (20-75\%). In two reviews (Koh 2020, Yanes-Lane 2020), studies judged to be of low quality were excluded from their meta-analyses. In one review (Chen 2020), the overall quality was reported as low, while $80 \%$ of included studies were reported as moderate or high quality in another two (Fung 2020, Madewell 2020). Another review (Chu 2020) reported the overall risk of bias as low-tomoderate, and one ( $\mathrm{Xu} 2020)$ rated the overall quality as low. Three reviews did not assess study quality (see Table 2).

\section{Primary studies}

We found 171 primary studies (Table 1). In general, the studies did not report any hypothesis but assessed epidemiological or mechanistic evidence for transmission associated with close contact settings. Ninety-three studies $(54.4 \%)$ were conducted in Asia, $43(25.1 \%)$ in Europe, 27 (15.8\%) in North America, five $(2.9 \%)$ in South America and three $(1.8 \%)$ in Australasia. The 


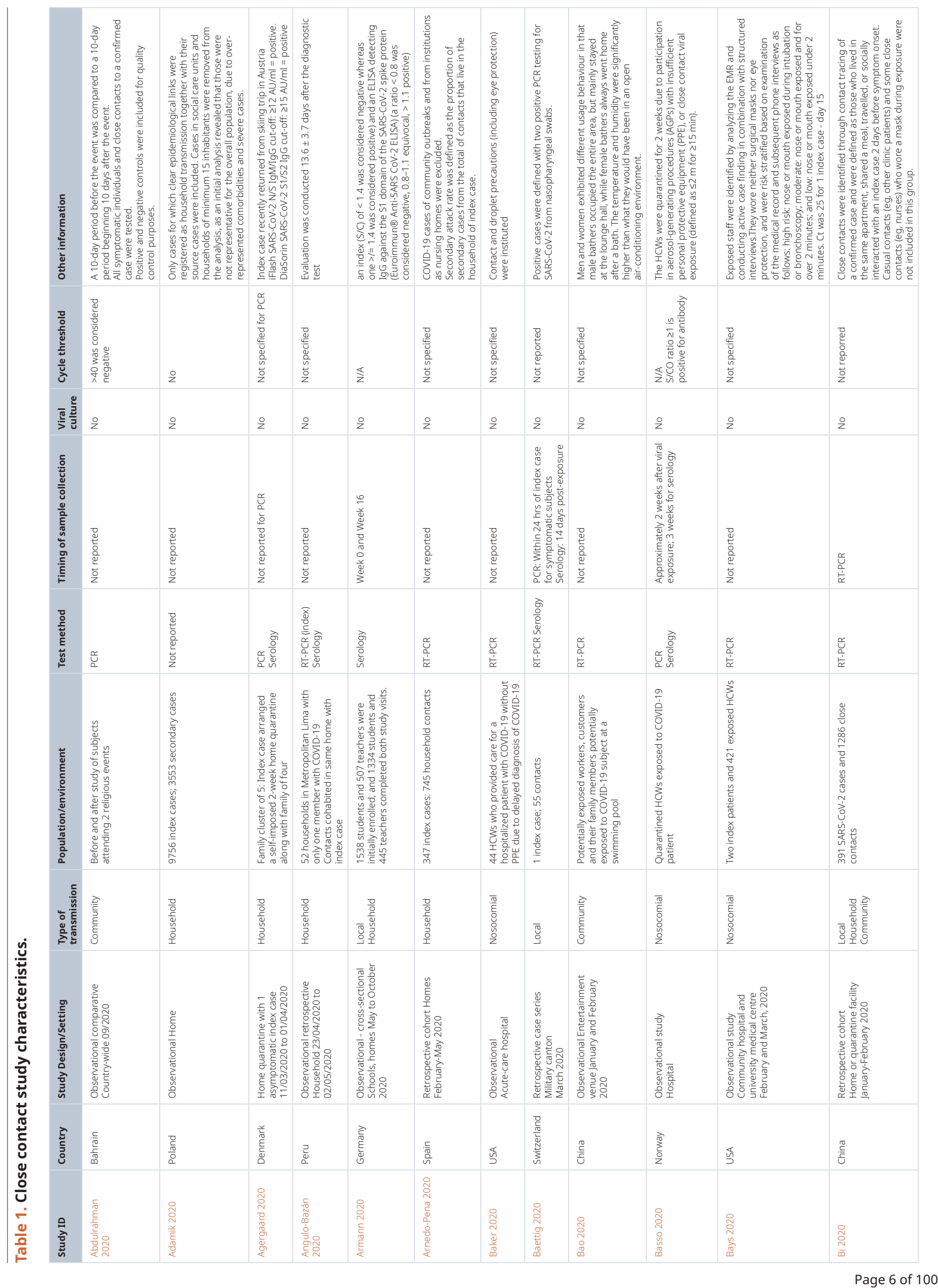




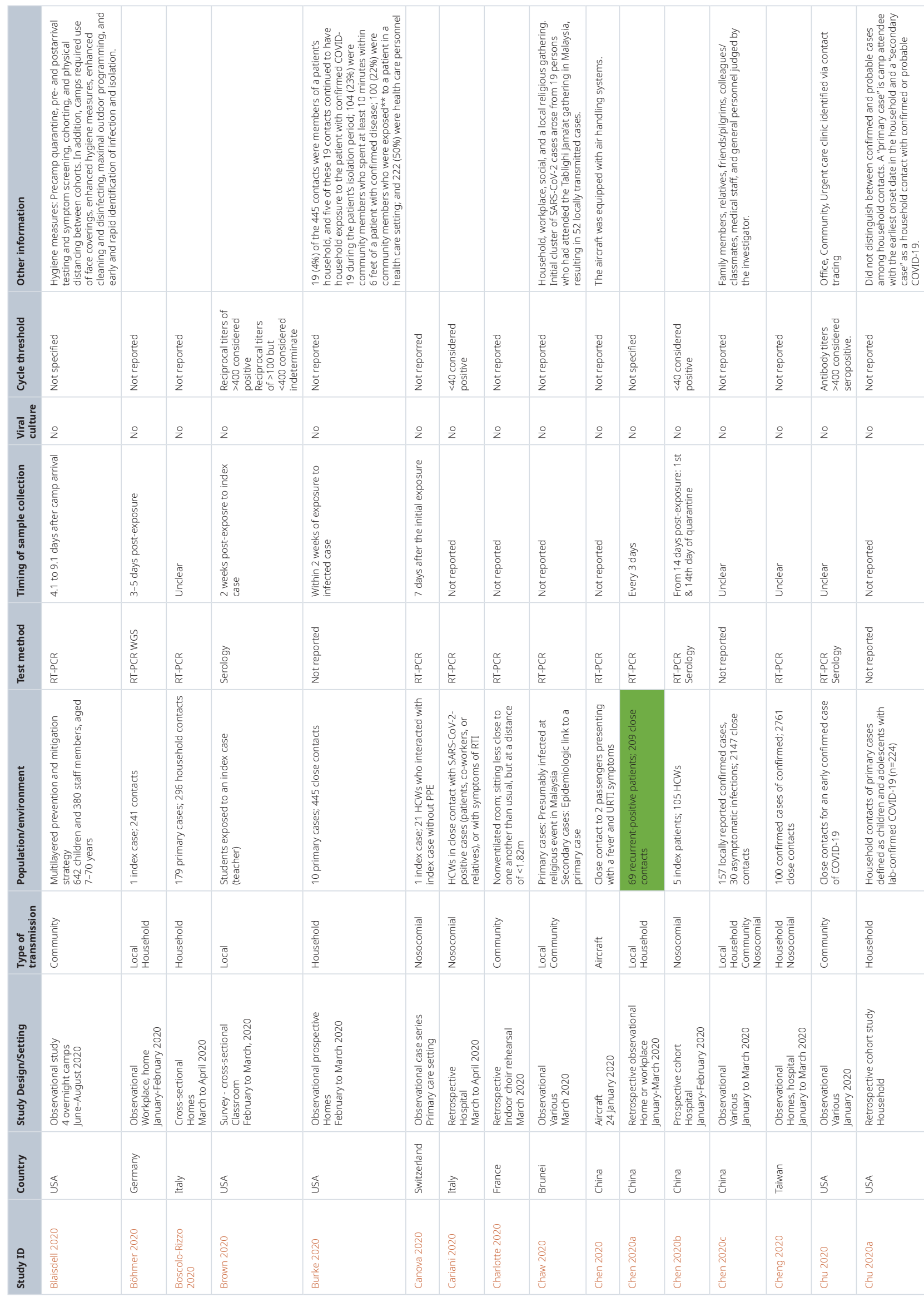




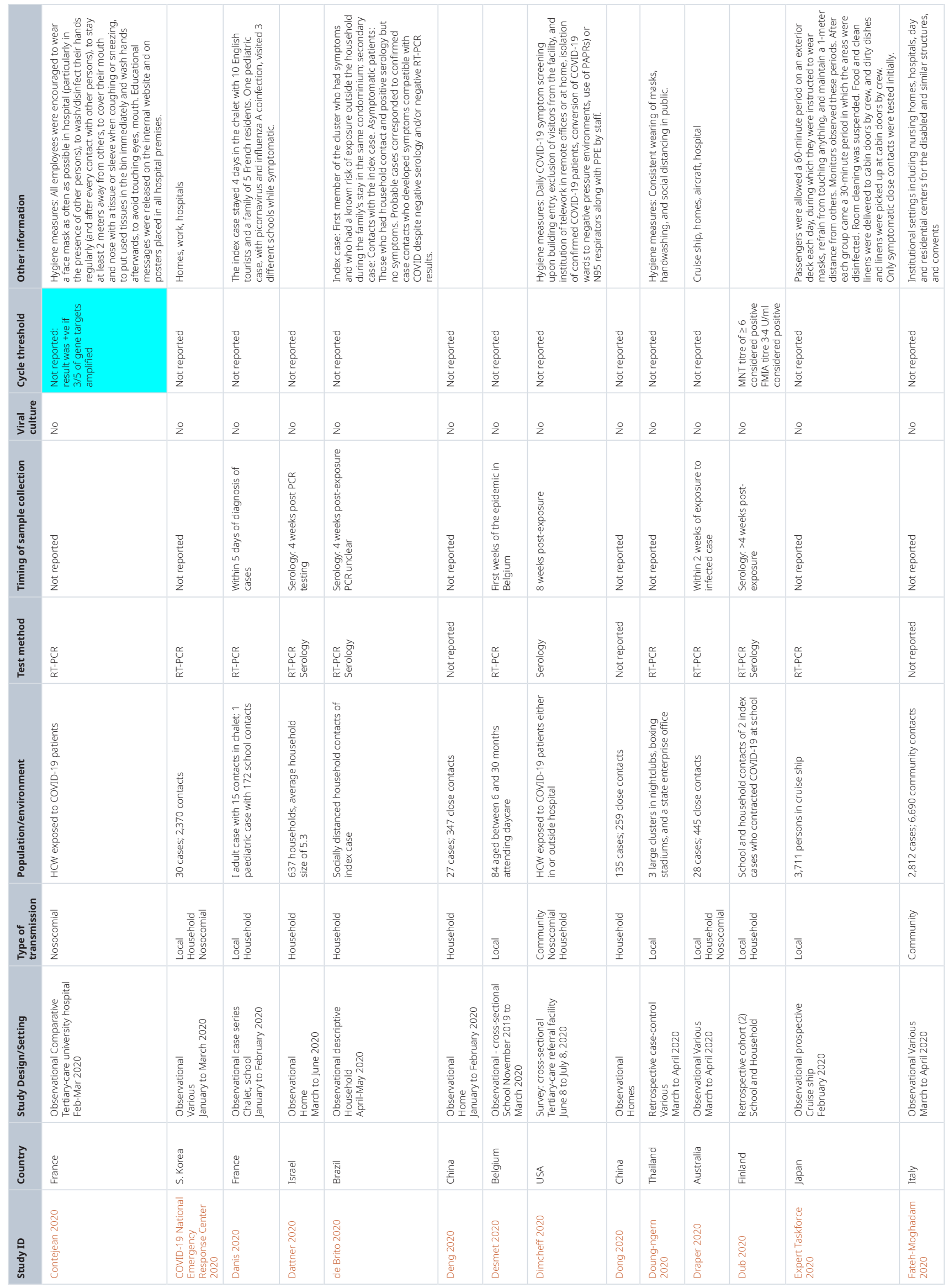




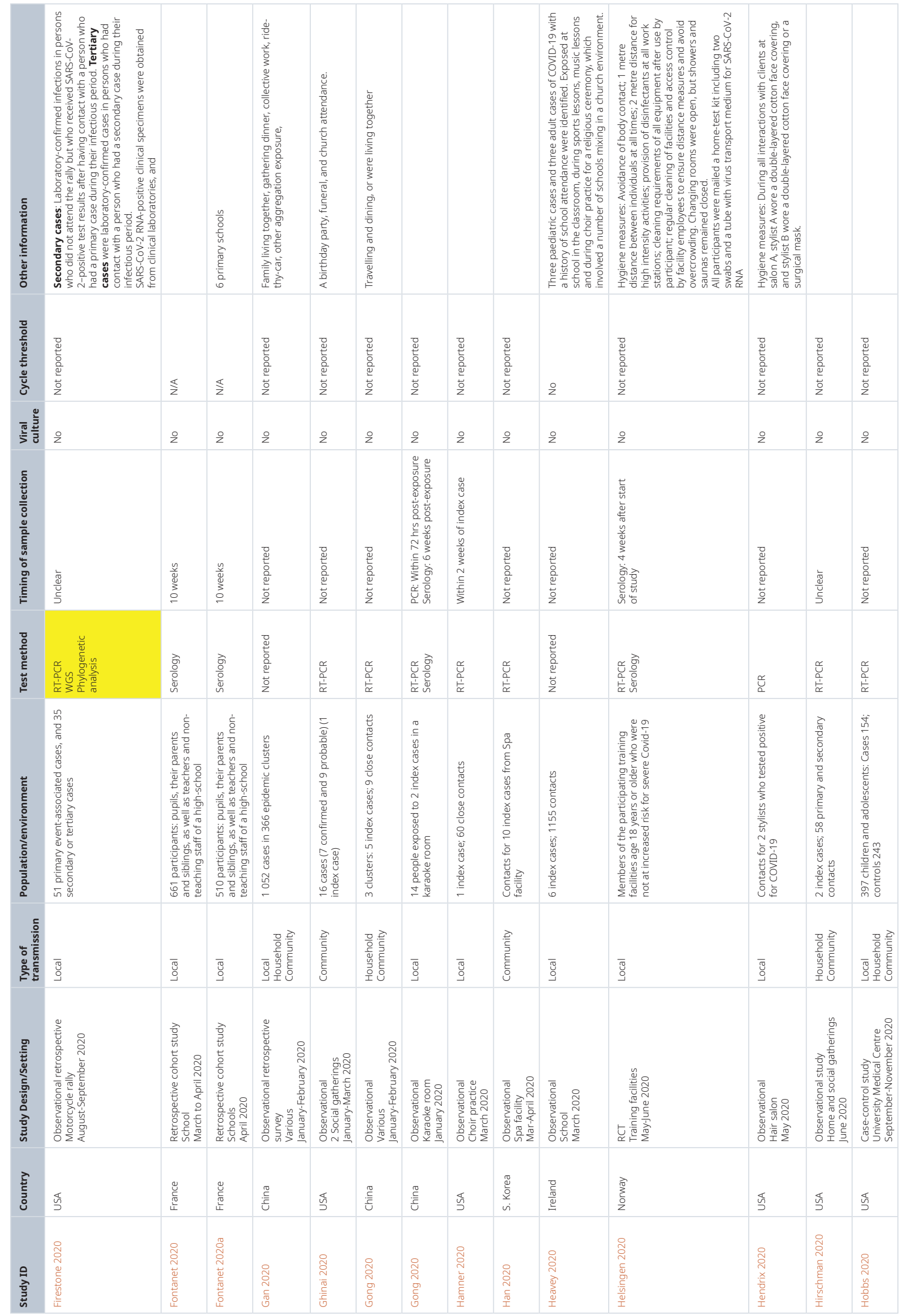




\begin{tabular}{|c|c|c|c|c|c|c|c|c|c|c|c|c|c|c|c|c|c|}
\hline 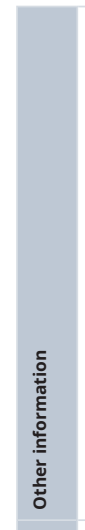 & 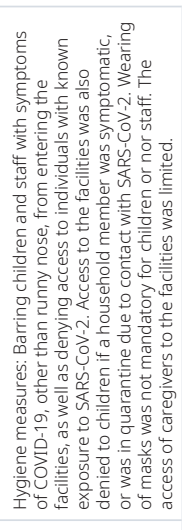 & & 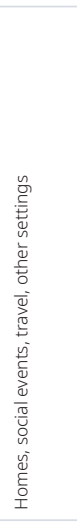 & & 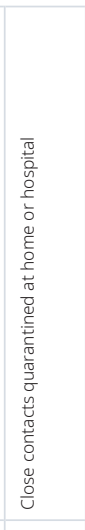 & 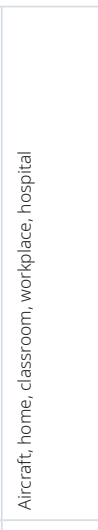 & 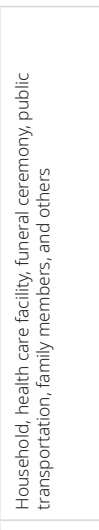 & 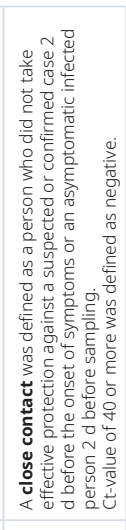 & 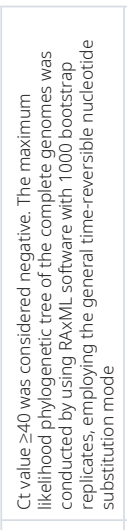 & & & 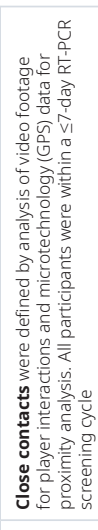 & & 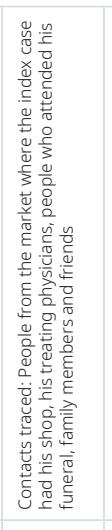 & 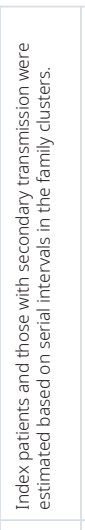 & 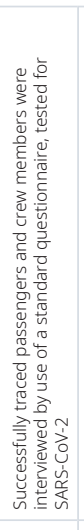 & 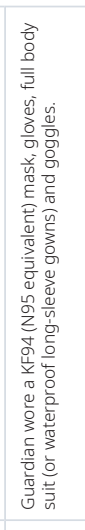 \\
\hline 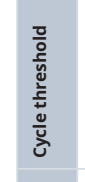 & 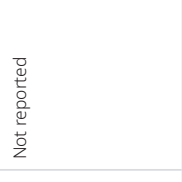 & 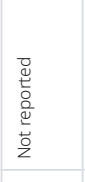 & 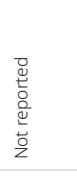 & 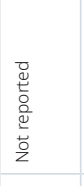 & 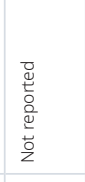 & 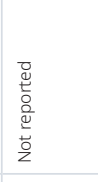 & 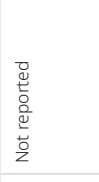 & 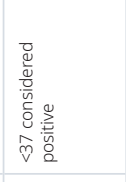 & 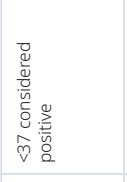 & 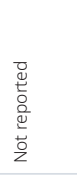 & 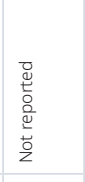 & 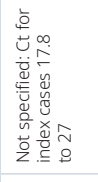 & 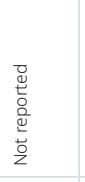 & 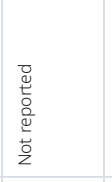 & 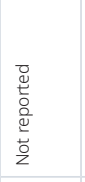 & 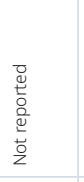 & 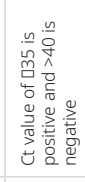 \\
\hline$\frac{\sqrt[0]{\frac{2}{2}}}{\frac{3}{3}}$ & $\stackrel{\circ}{z}$ & $\stackrel{\circ}{z}$ & $\stackrel{0}{2}$ & $\stackrel{0}{2}$ & $\stackrel{0}{2}$ & $\stackrel{\circ}{2}$ & qo & $\stackrel{0}{z}$ & q & $\stackrel{\circ}{2}$ & $\stackrel{0}{2}$ & $\stackrel{\circ}{2}$ & $\stackrel{0}{z}$ & qo & qo & $\stackrel{\circ}{z}$ & $\stackrel{\circ}{z}$ \\
\hline 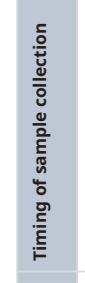 & 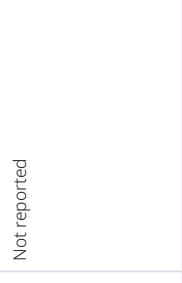 & 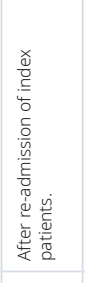 & 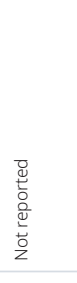 & 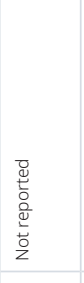 & 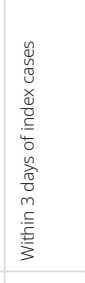 & 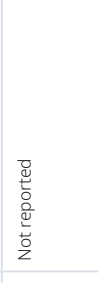 & 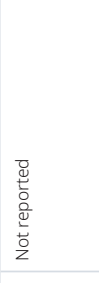 & 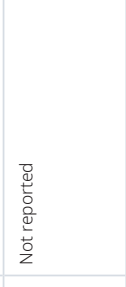 & 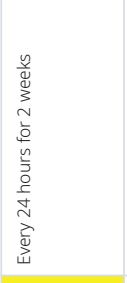 & 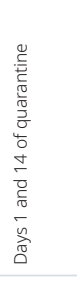 & 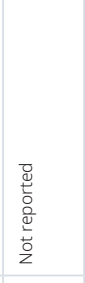 & 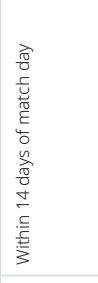 & 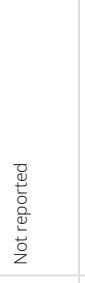 & 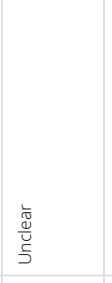 & 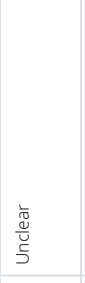 & 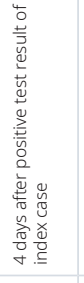 & 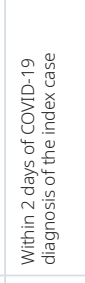 \\
\hline 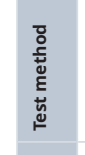 & 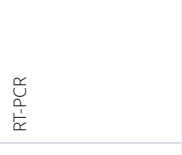 & 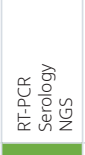 & 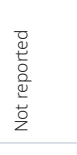 & 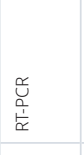 & 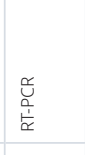 & 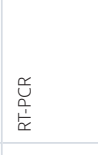 & 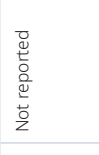 & 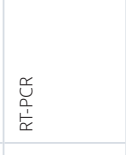 & 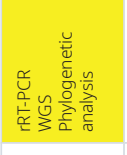 & 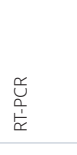 & $\begin{array}{l}\text { 总 } \\
\text { 趸 } \\
\text { L }\end{array}$ & 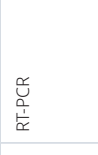 & 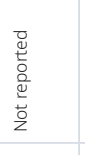 & 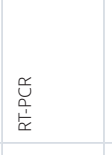 & 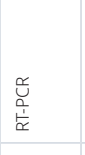 & $\stackrel{q}{a}$ & 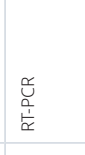 \\
\hline 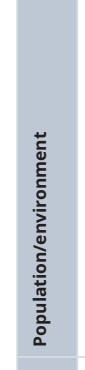 & 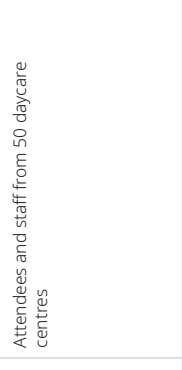 & & 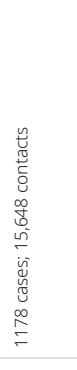 & 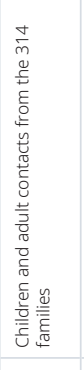 & 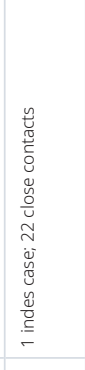 & 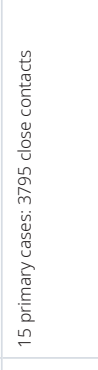 & 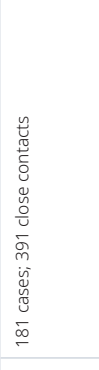 & 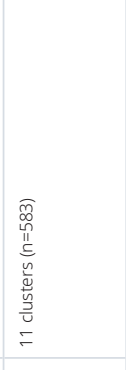 & 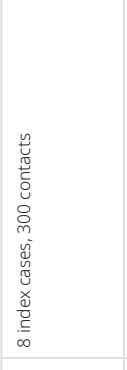 & 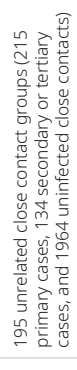 & 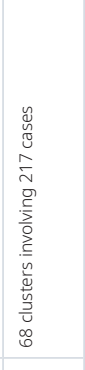 & 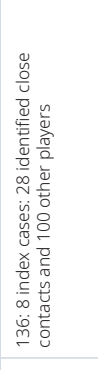 & 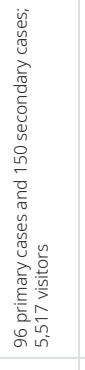 & 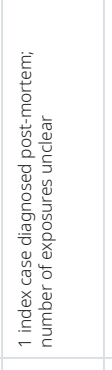 & 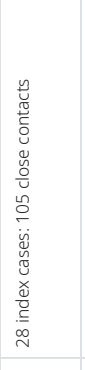 & 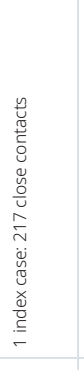 & 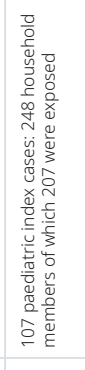 \\
\hline 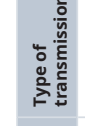 & 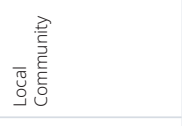 & 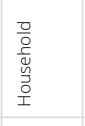 & 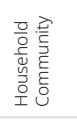 & 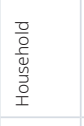 & 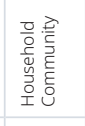 & 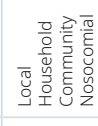 & 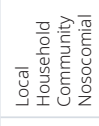 & 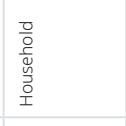 & 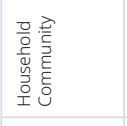 & 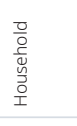 & 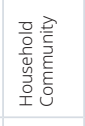 & 㲛 & 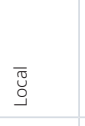 & 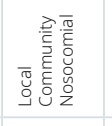 & 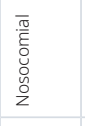 & & 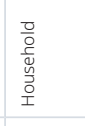 \\
\hline 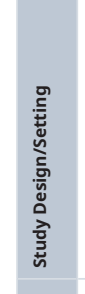 & 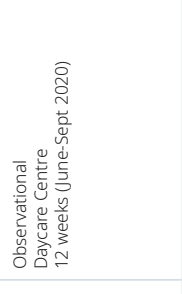 & 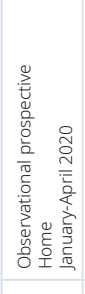 & 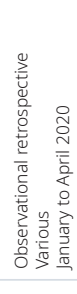 & 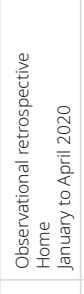 & 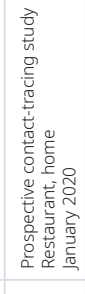 & 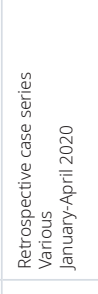 & 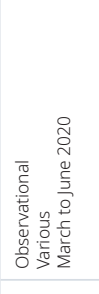 & 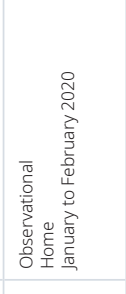 & 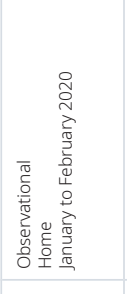 & 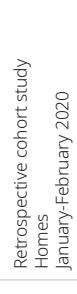 & 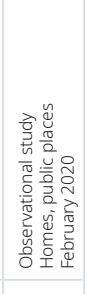 & 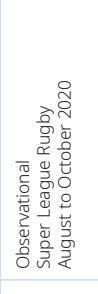 & 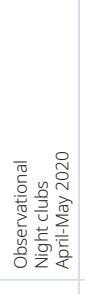 & 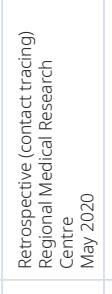 & 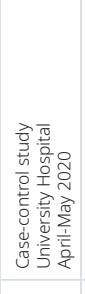 & 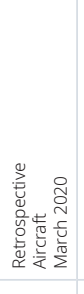 & 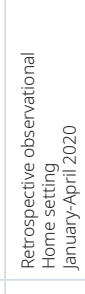 \\
\hline 。气 & 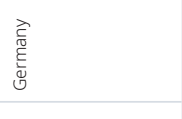 & 营 & 离 & 离 & 苞 & 离 & 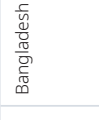 & 总 & 苟 & 旁 & 总 & 弚总 & 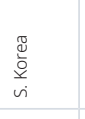 & 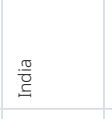 & 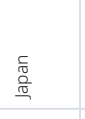 & 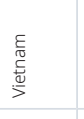 & 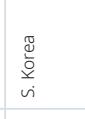 \\
\hline 突 & 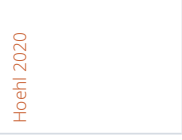 & 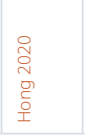 & 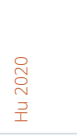 & 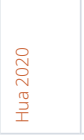 & 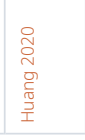 & 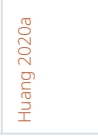 & 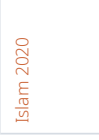 & $\begin{array}{l}\text { ב̃ } \\
\stackrel{N}{=}\end{array}$ & 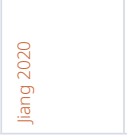 & 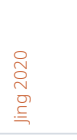 & 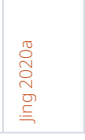 & 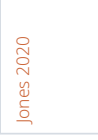 & 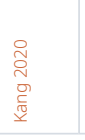 & 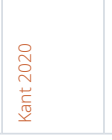 & 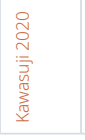 & 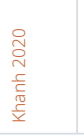 & న్ \\
\hline
\end{tabular}




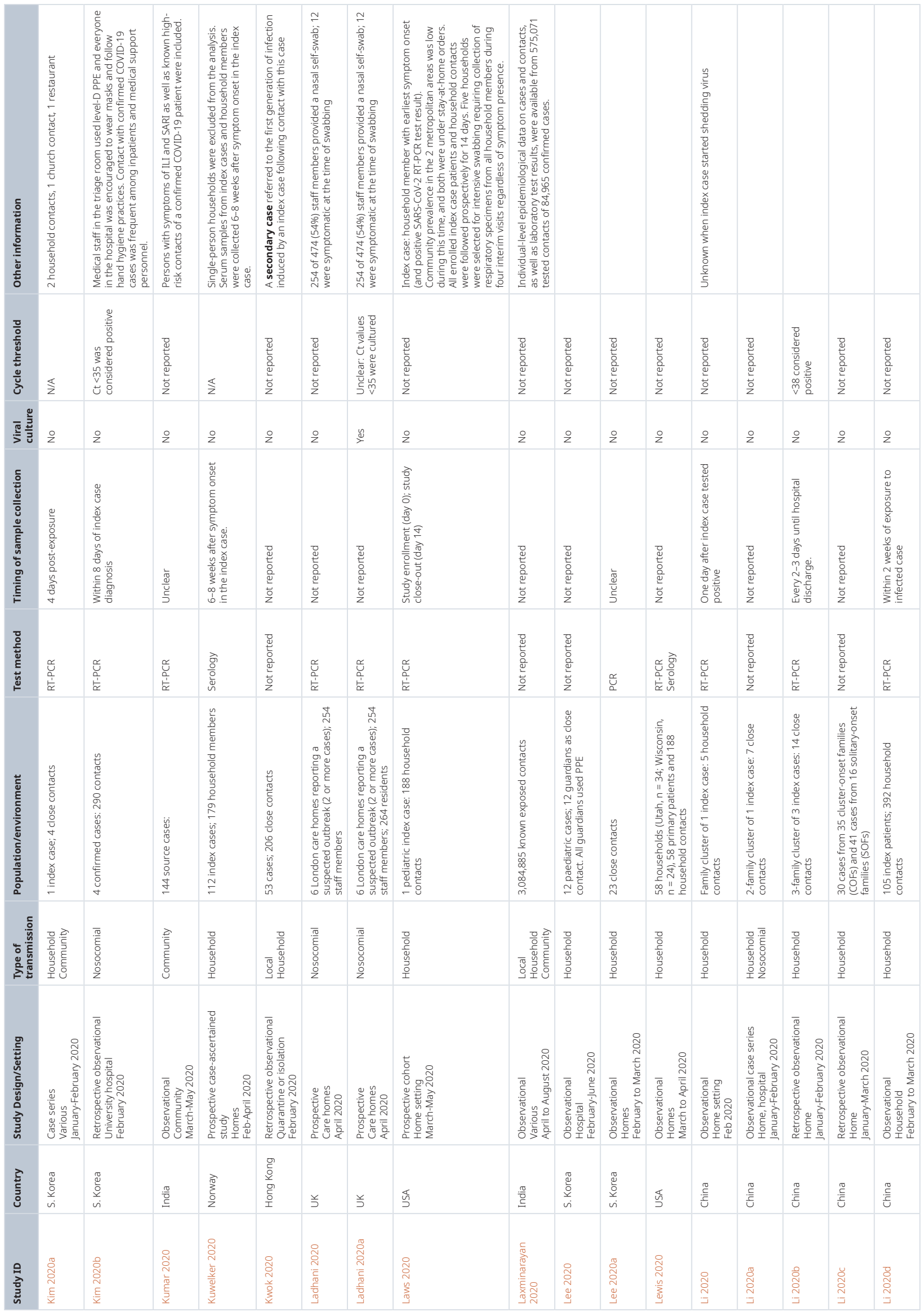




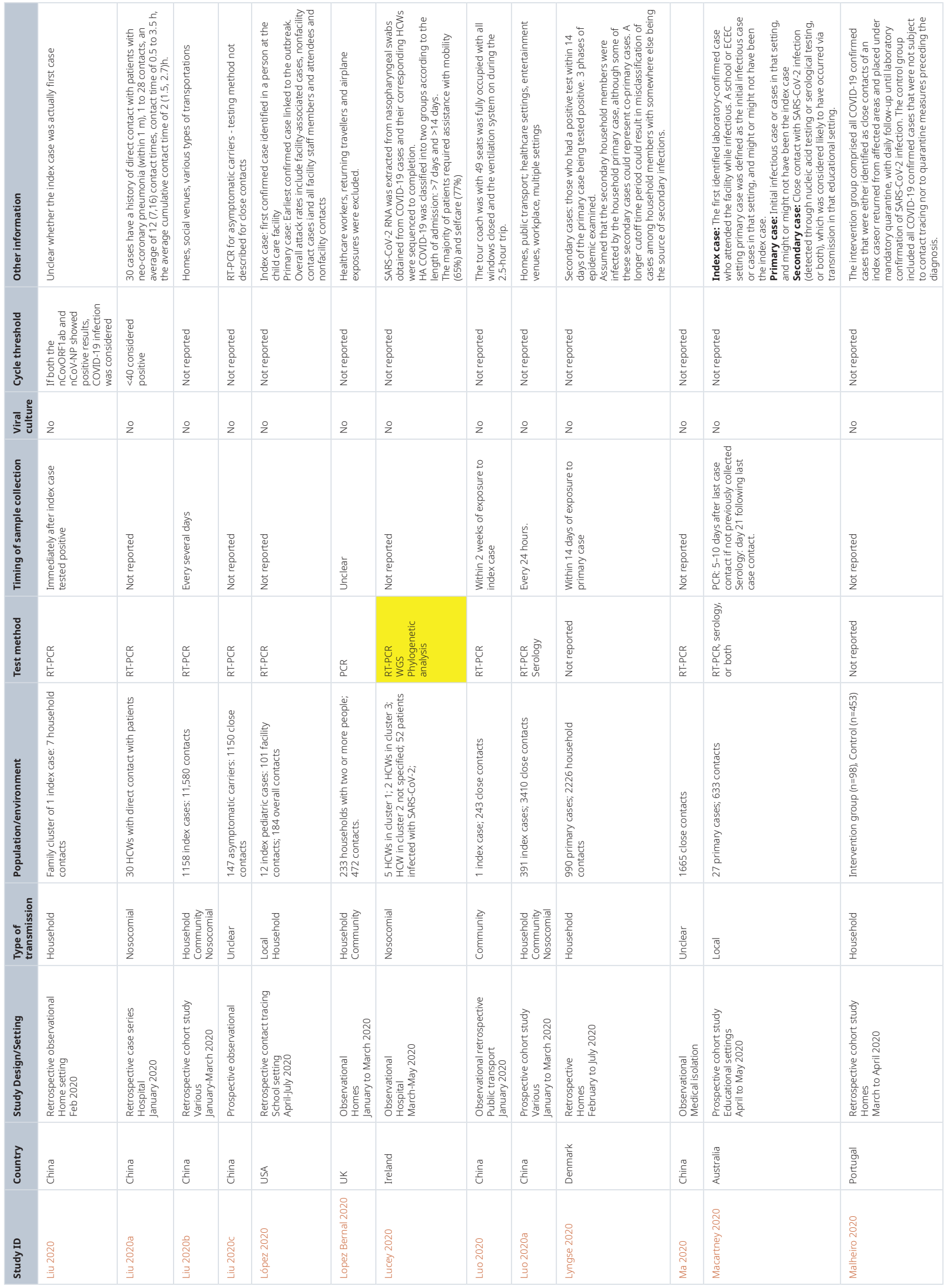




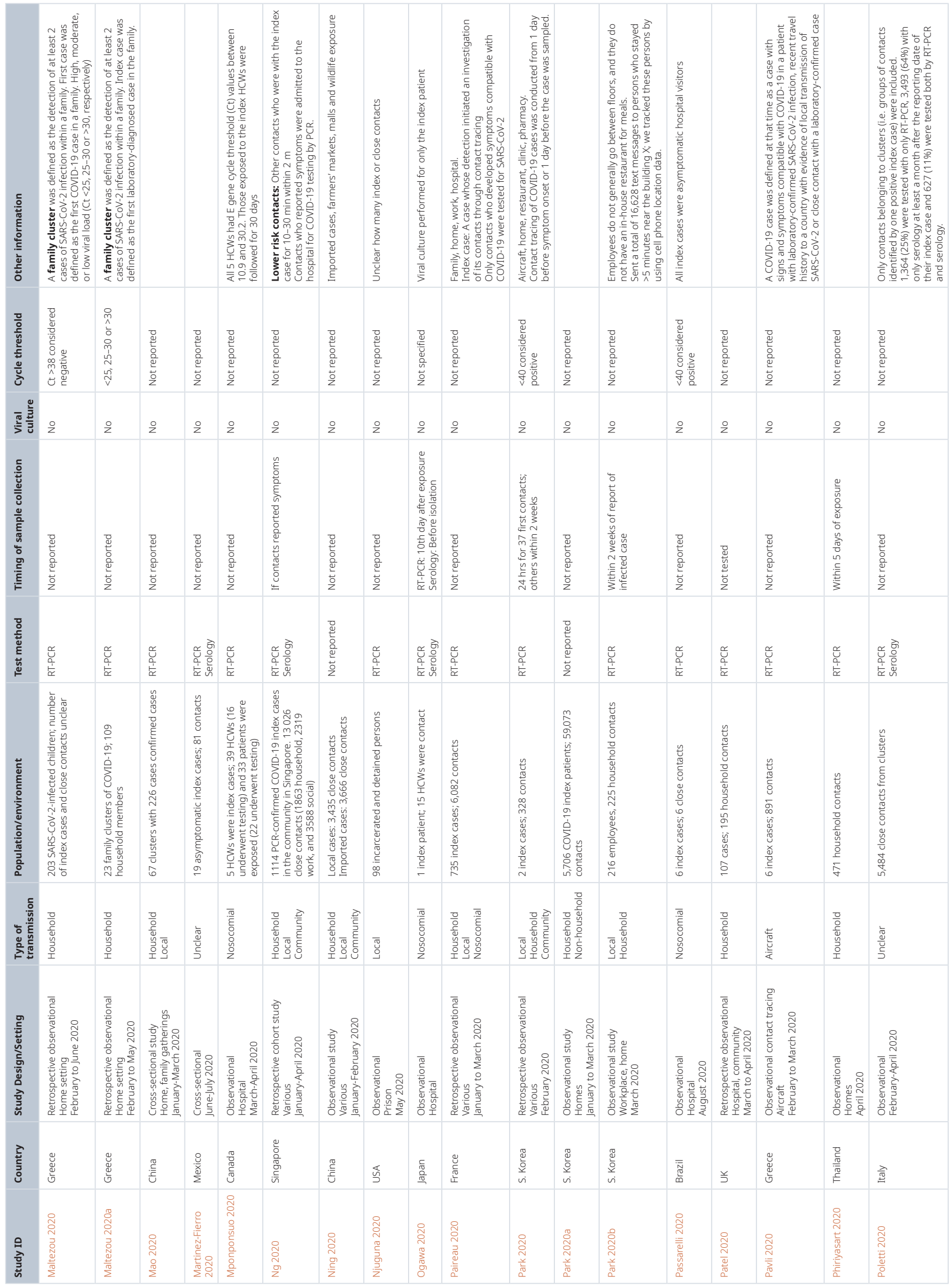




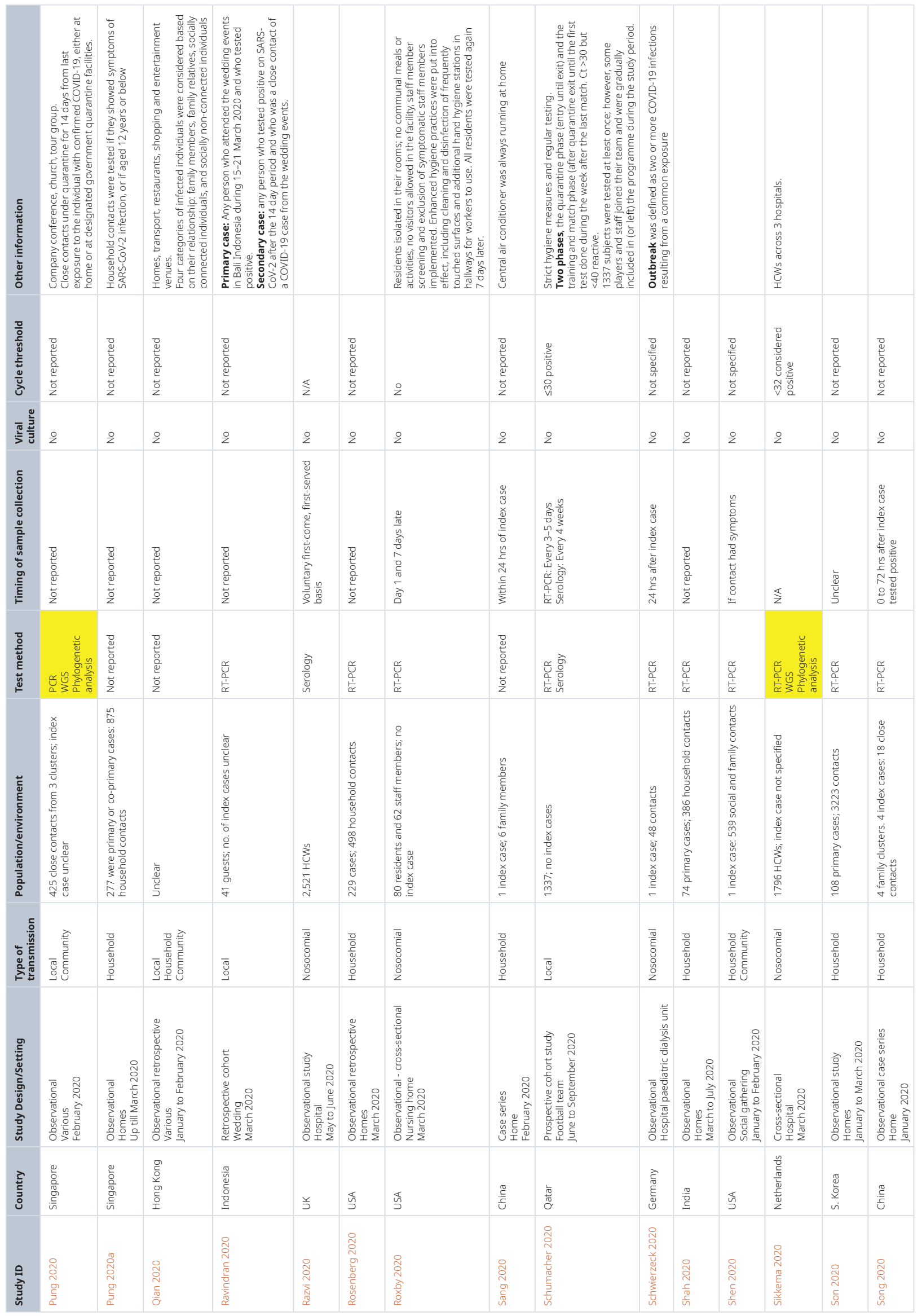




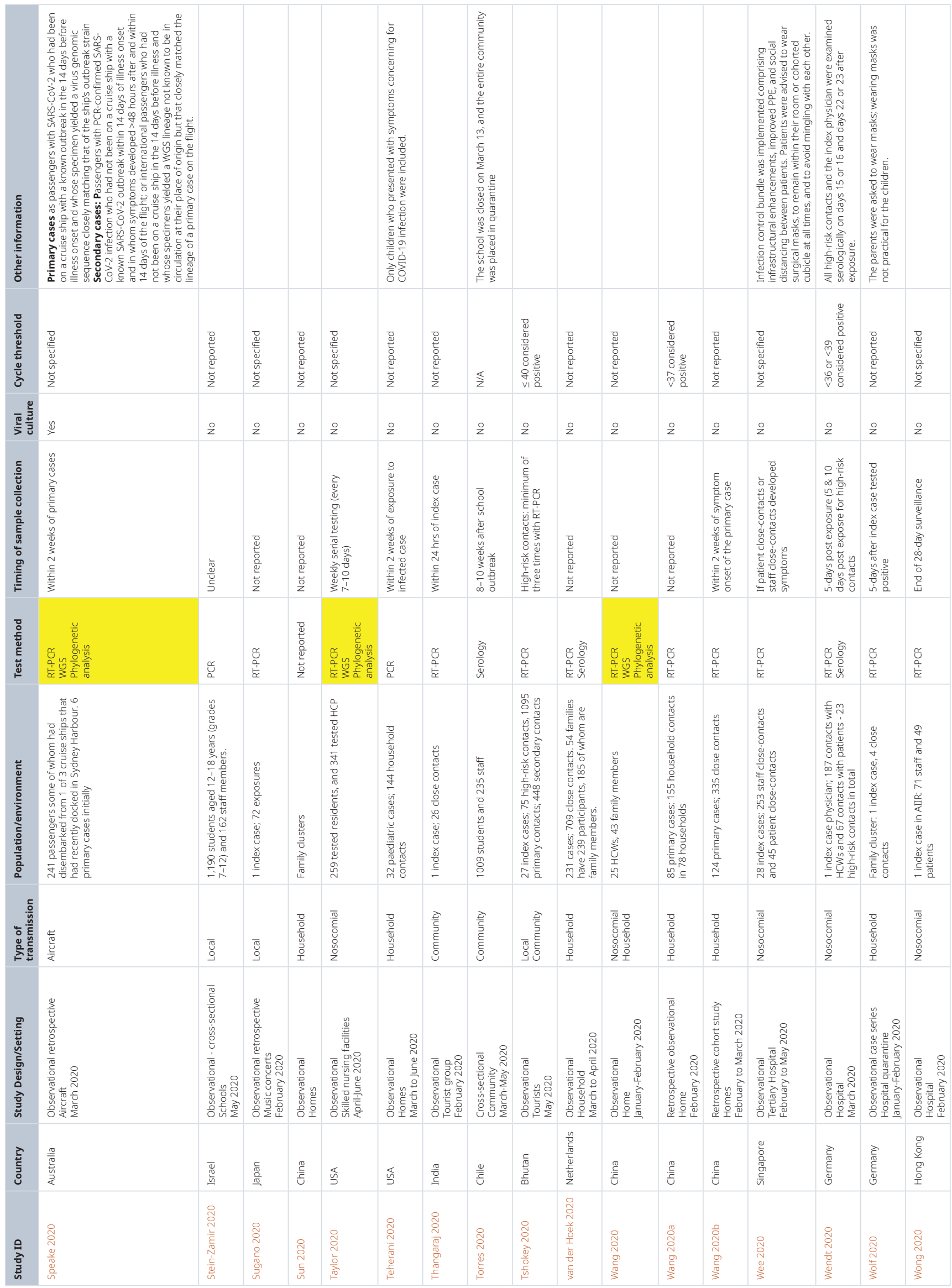




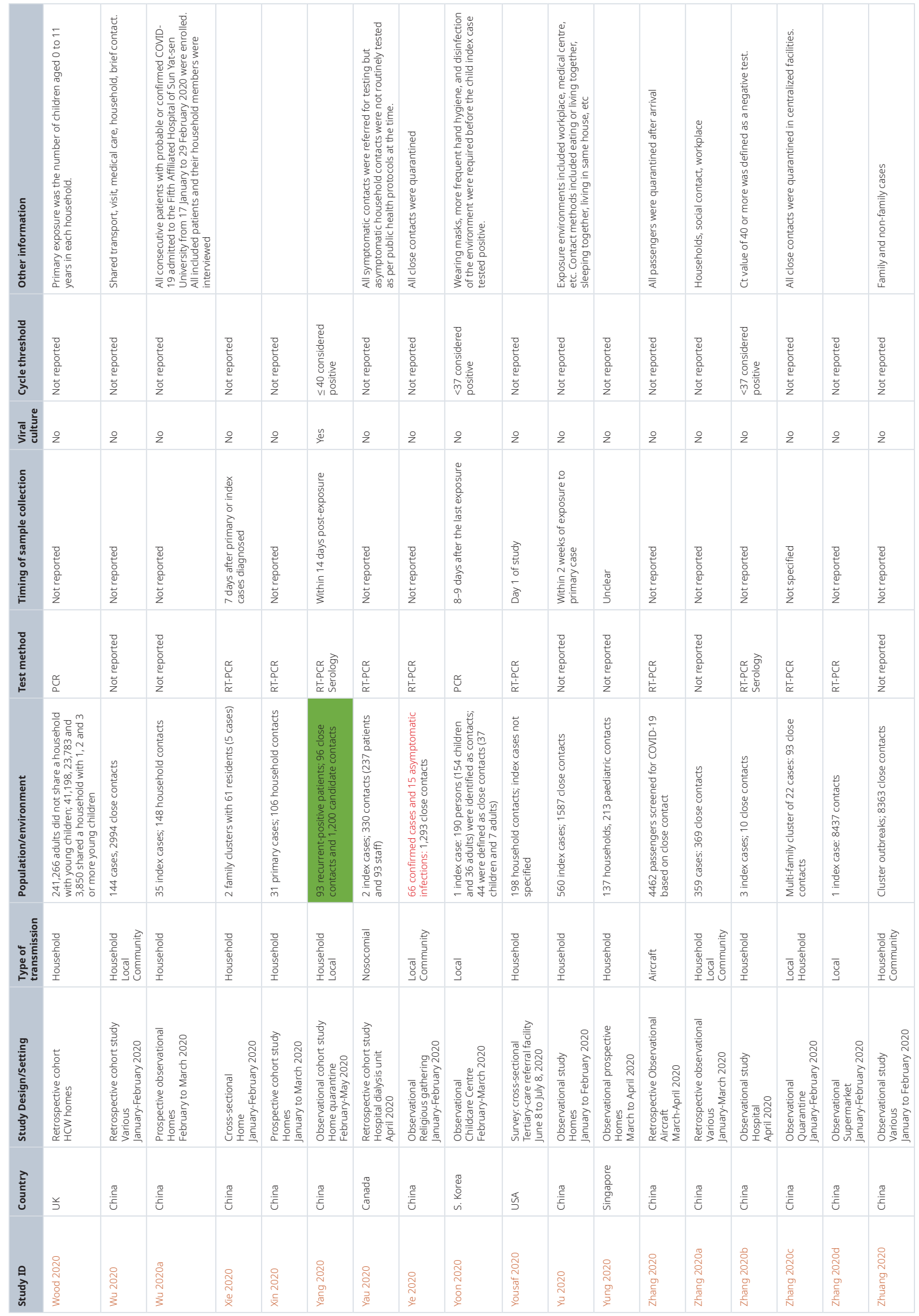




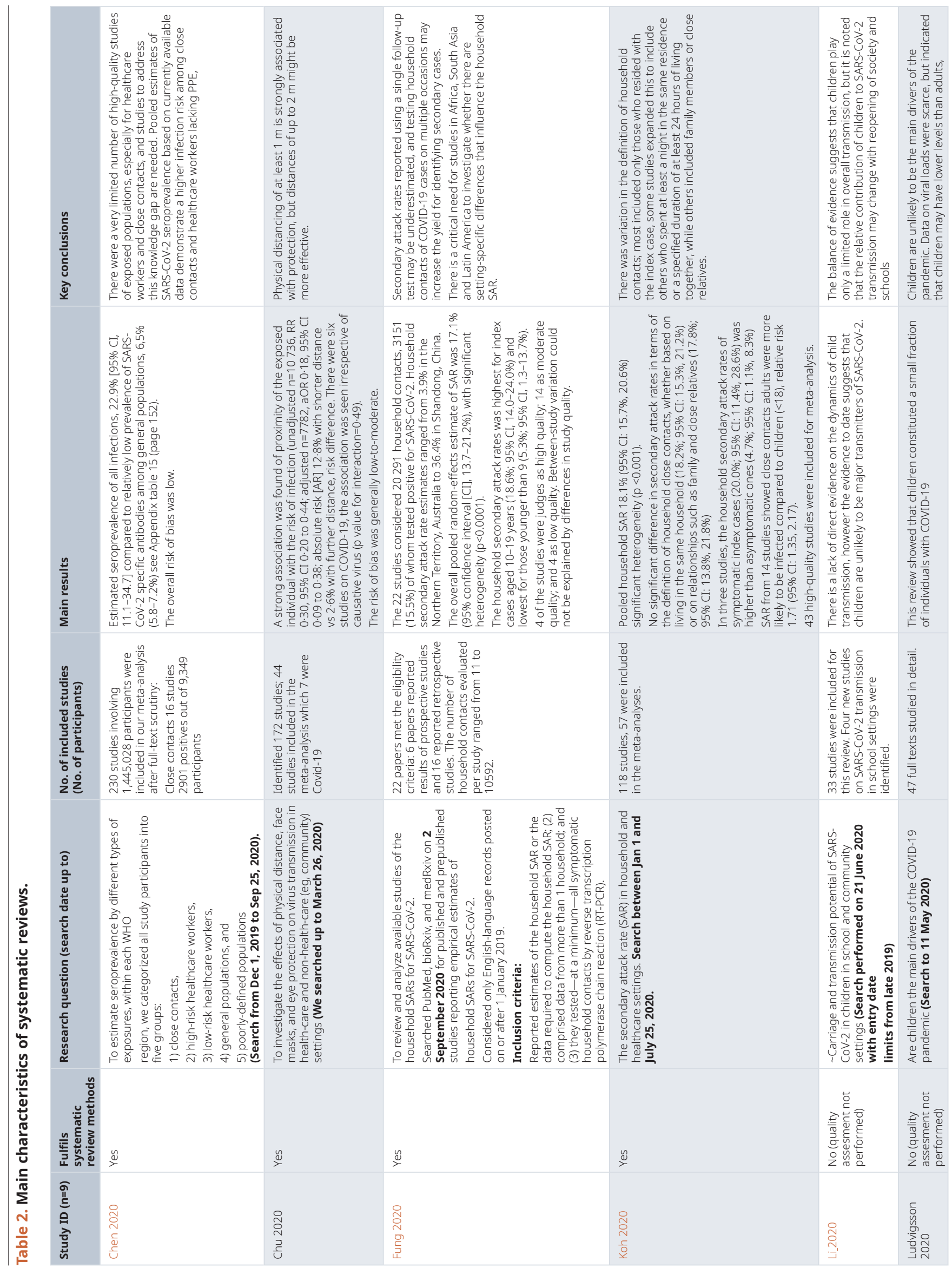




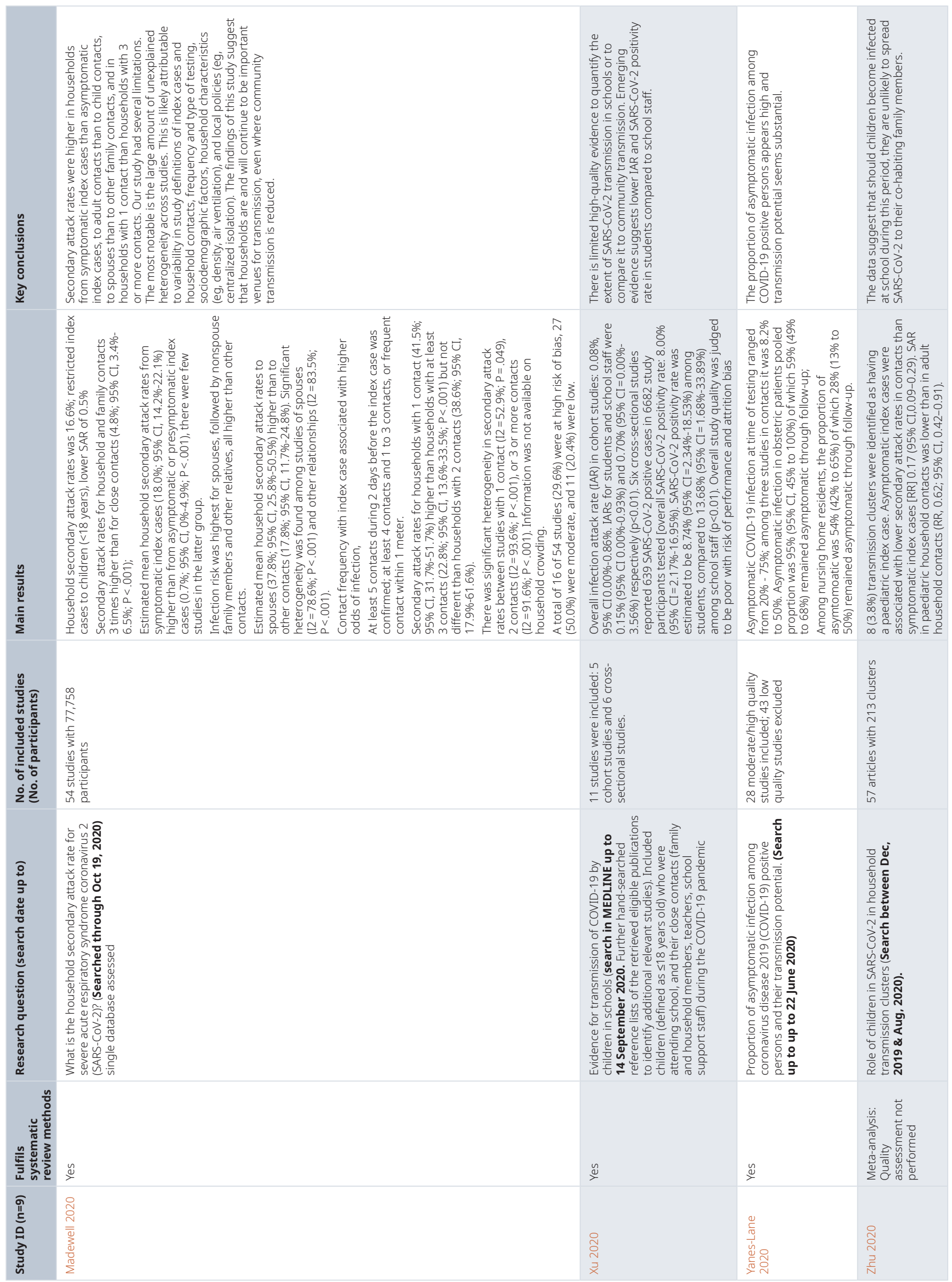




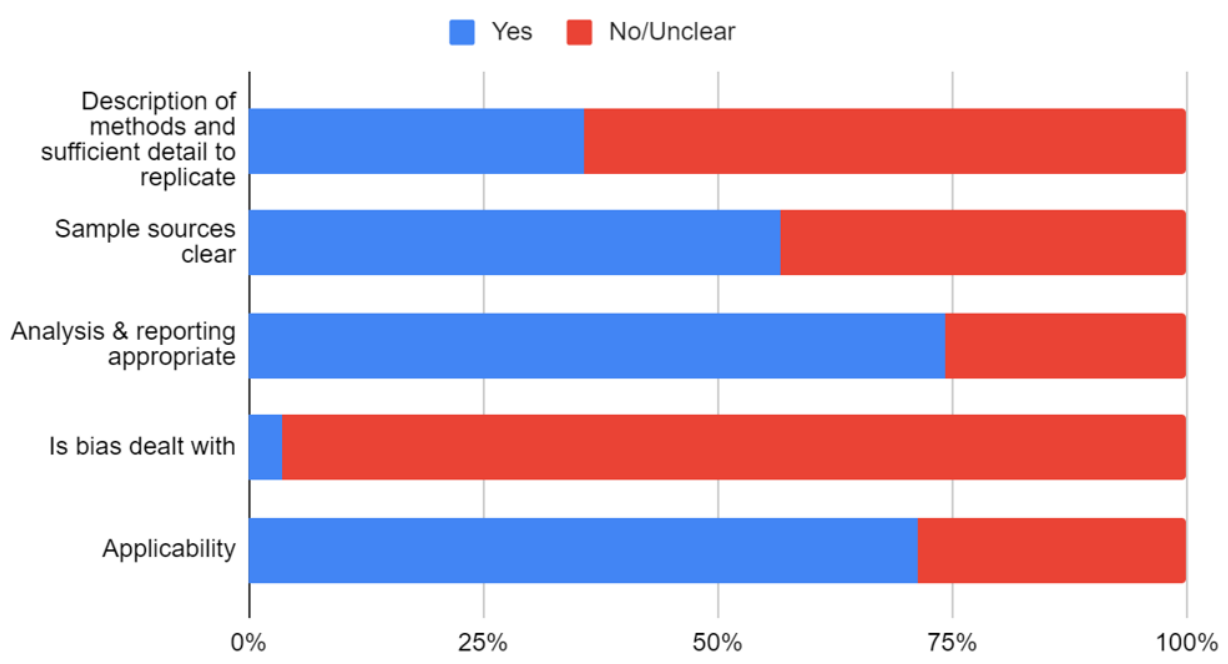

Figure 2. Risk of bias graph in primary studies of close contacts in SARS-CoV-2.

study settings included home/quarantine facilities $(n=54)$, hospital $(n=26)$, social/religious gatherings $(n=13)$, public transport $(n=7)$ care homes $(n=4)$, and educational settings $(n=8)$. Thirteen studies used two settings (home plus one other setting). In 25 studies $(15.2 \%)$, the settings were multiple (3 or more different settings). Two studies were conducted in professional sports settings: one Super League Rugby (Jones 2020) and one football team (Schumacher 2020)

All the included studies were observational in design except one RCT (Helsingen 2020): 24 studies were described as cohort, nine were case series and 12 cross-sectional. One study used a before and after study design. The number of close contact participants included ranged from 4 to 8437 . Three studies (Chen 2020a, Hong 2020, Yang 2020) examined transmission dynamics in close contacts of index or primary cases with recurrent SARS-CoV-2 infections.

Eighty-two studies (46.8\%) reported definitions of close contacts (Table 3 ). There was a variation in the definitions across the studies. Seventeen studies $(9.9 \%)$ defined close contact as exposure to the index or primary case within two metres for at least 15 minutes while four defined it as being within $2 \mathrm{~m}$ for at least 10 minutes. In 24 studies, there was no specified distance reported - close contact definitions included unprotected exposure, living in the same household or bedroom, sharing a meal, or having repeated and prolonged contact. In five studies of airline passengers, close contact was defined as all passengers on the flight (Chen 2020), seated within two rows of the index case (Draper 2020, Pavli 2020, Speake 2020), or being within $2 \mathrm{~m}$ for at least 15 minutes (Khanh 2020). Eighty-seven studies $(50.9 \%)$ did not define close contact and the definition was unclear in four studies. Twenty-nine studies (17\%) defined other types of contacts including primary contact, secondary contact, high-risk contact, household contact, social contact, and work contact (see Table 3).

Eighteen studies (10.5\%) reported data on the contact duration between close contacts and the index or primary cases (Table 3). The average contact duration ranged from 30 minutes to 8 days across 16 studies that investigated transmission rates using RT-PCR. In two studies that examined transmission using serology (Agergaard 2020, Hong 2020), the durations of contact were two weeks and 258 person-days, respectively. The mean contact duration was either unclear or not reported in 148 studies $(90.2 \%)$.

A total of 110 studies (64.3\%) used RT-PCR as a test method for confirming SARS-CoV-2 positivity, while eight studies (4.8\%) exclusively investigated transmission using serology. In 24 studies (14\%), both PCR and serology were used to investigate close contact in SARS-CoV-2 transmission. Thirty-one studies $(18.1 \%)$ did not report the test method used. For PCR, the timing of sample collection varied from within 24 hours to 14 days after exposure to the index or primary case; for serology, this ranged from $2-10$ weeks post-exposure. In total, 71 studies $(41.5 \%)$ reported the timing of sample collection. The timing of sample collection was either not reported or unclear in 100 studies $(58.5 \%)$.

Twenty-two studies (12.9\%) reported $\mathrm{Ct}$ values for determining PCR test positivity: $\leq 40$ (eight studies), <37 (five studies), $\leq 35$ (three studies), $<38$ (two studies), one each for $<25, \leq 30,<32$ and $<36$ (or 39). Only eight studies reported the $\mathrm{Ct}$ values for close contacts in their results - these ranged from 16.03 to 38 .

Thirty-two studies reported conducting serological tests to assess transmission of SARS-CoV-2 (Table 4). There was variation 


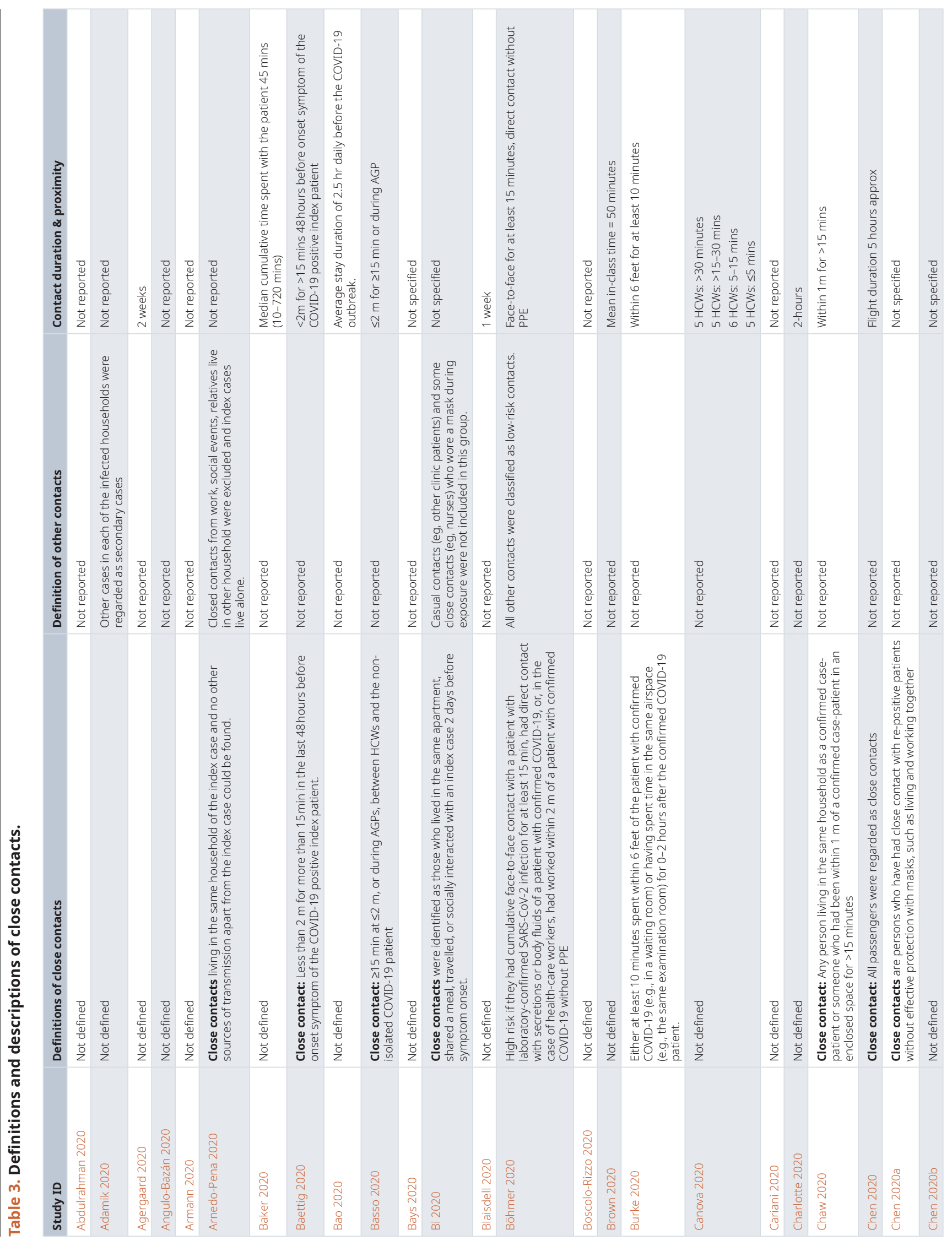




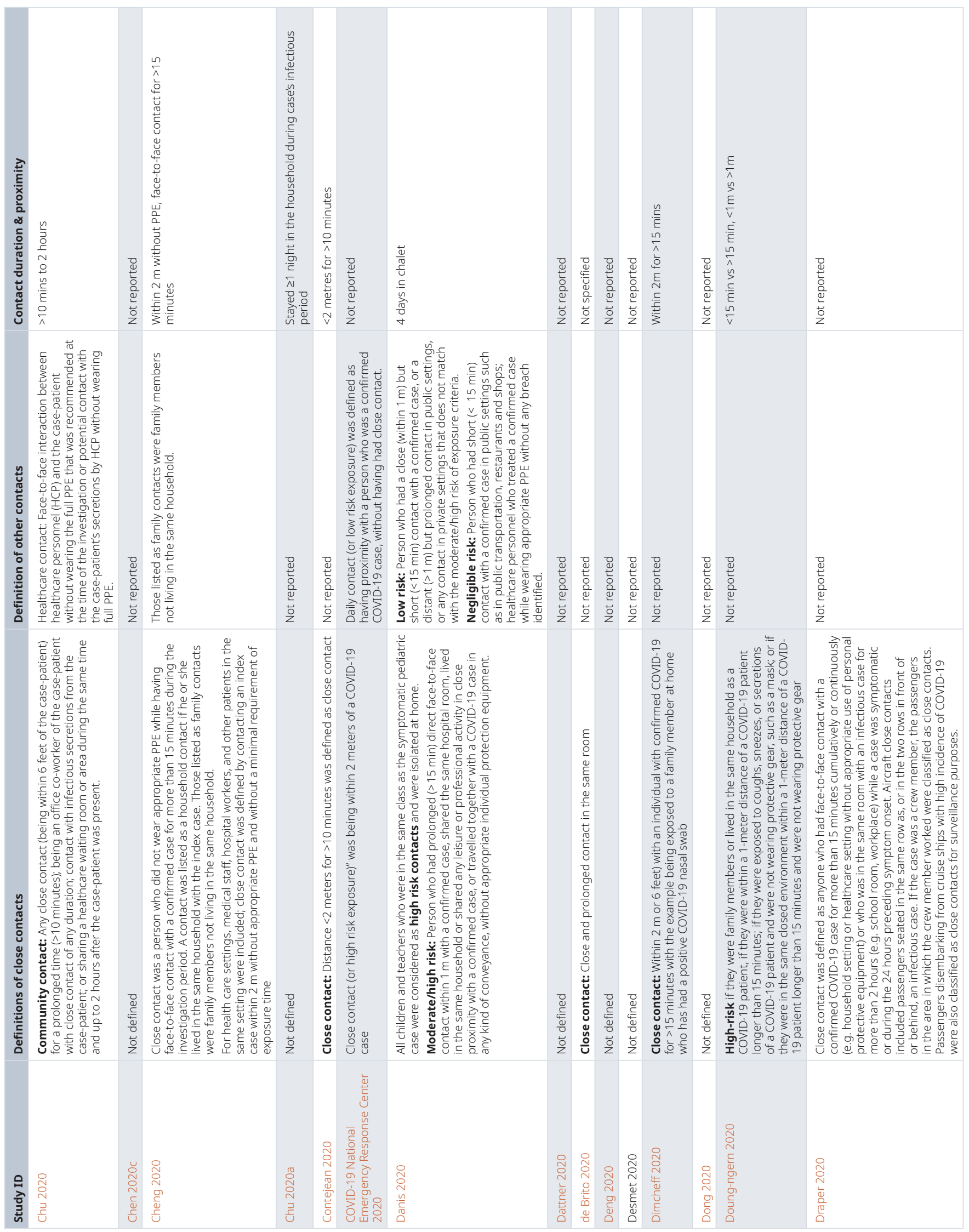




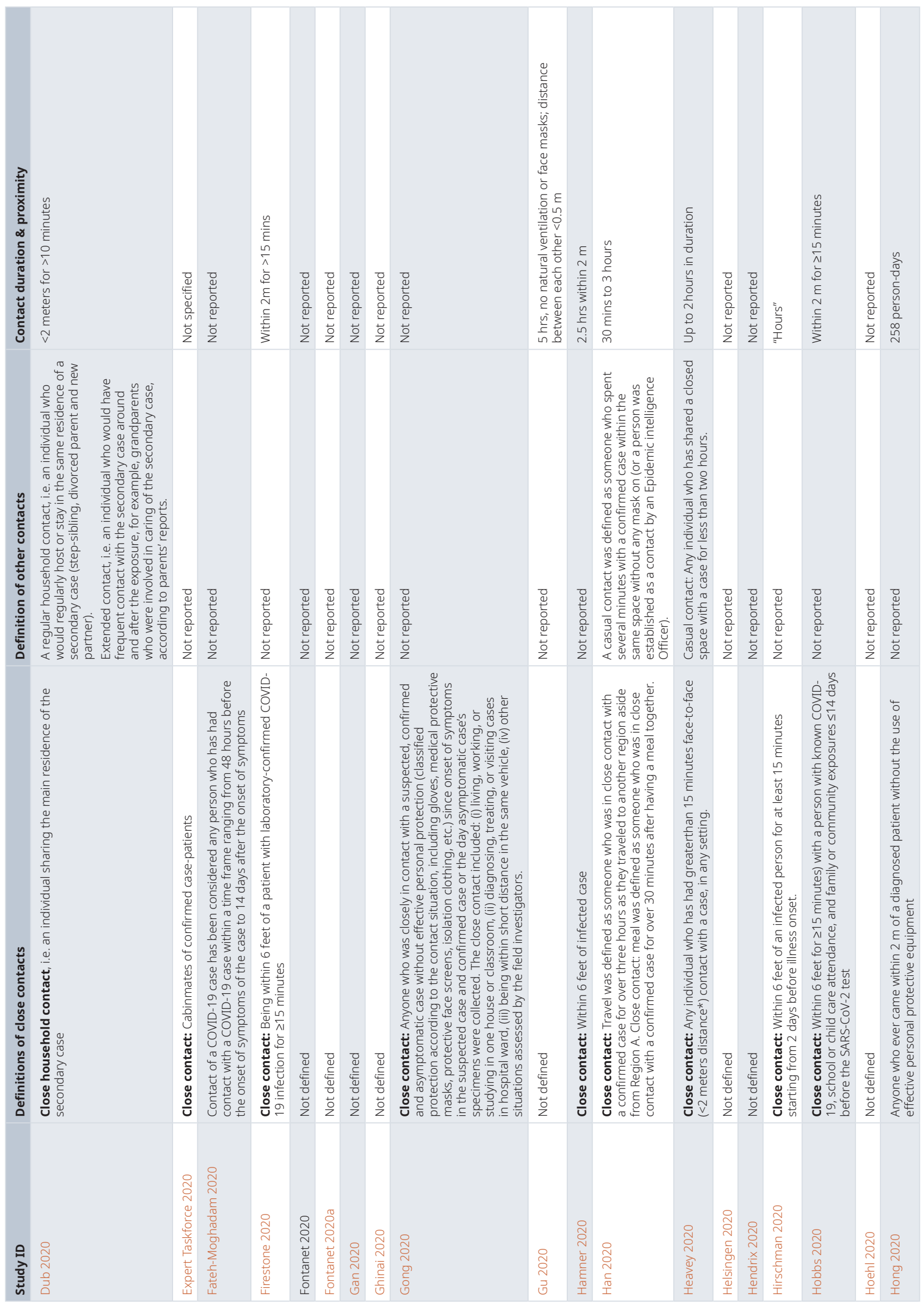




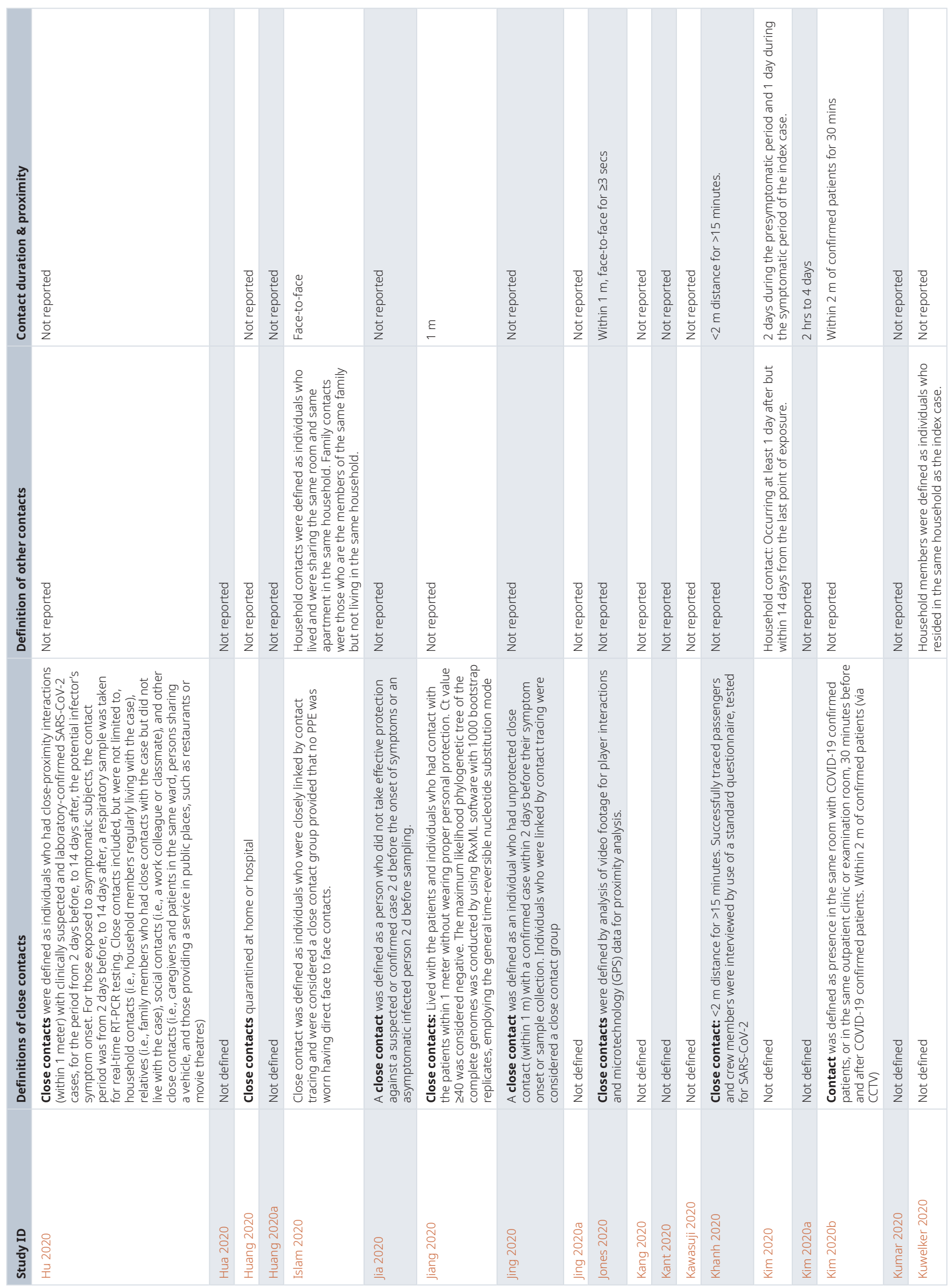




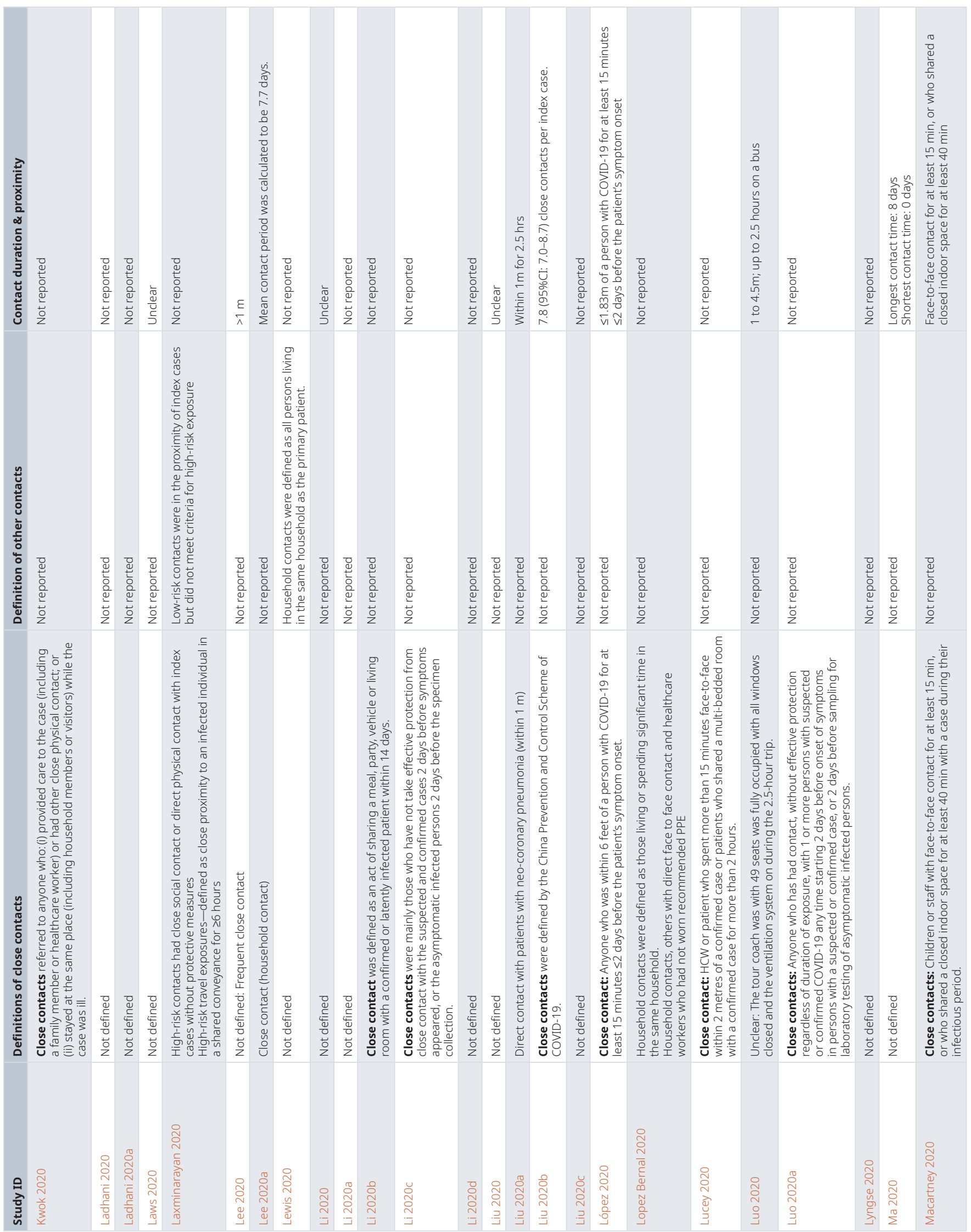




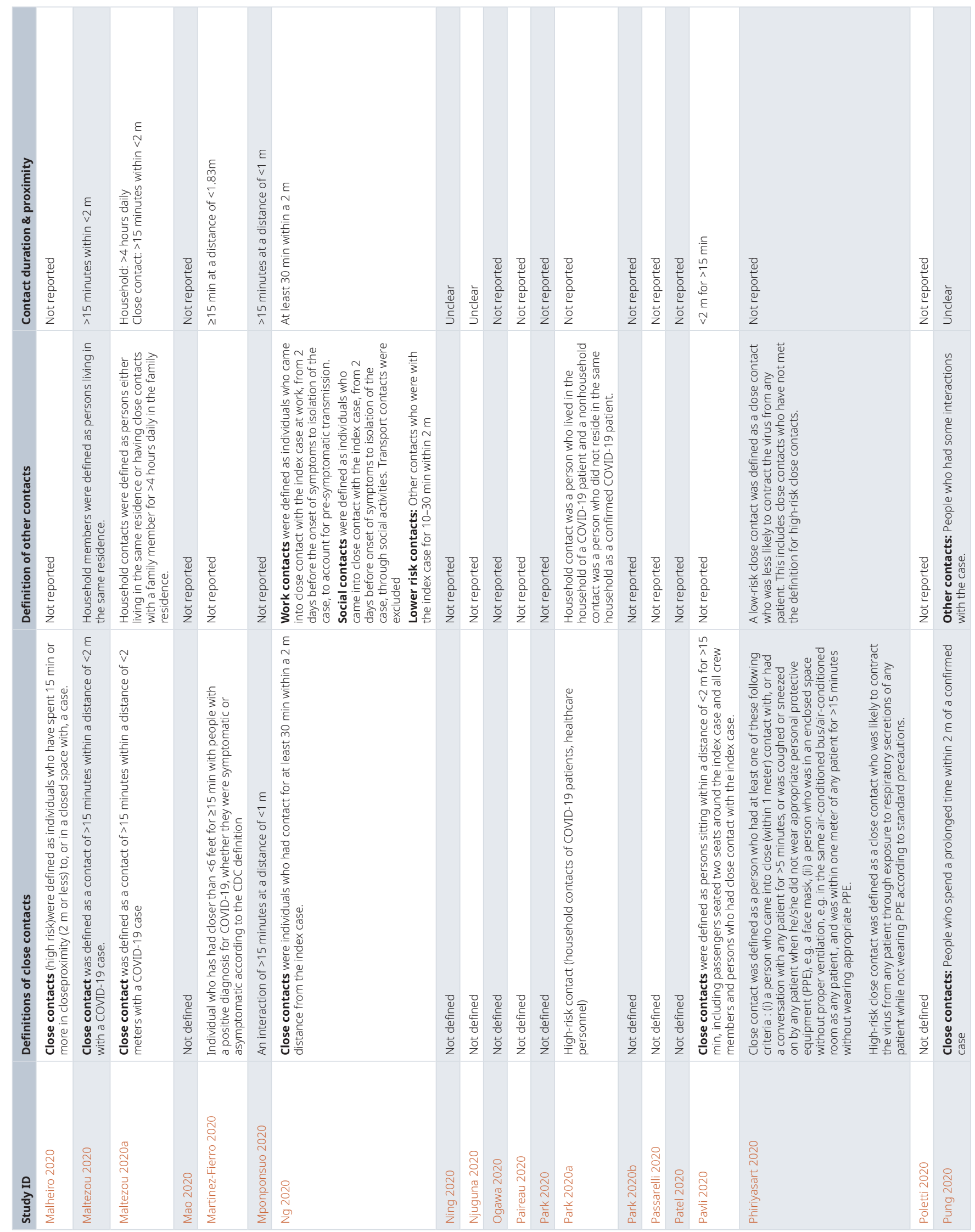




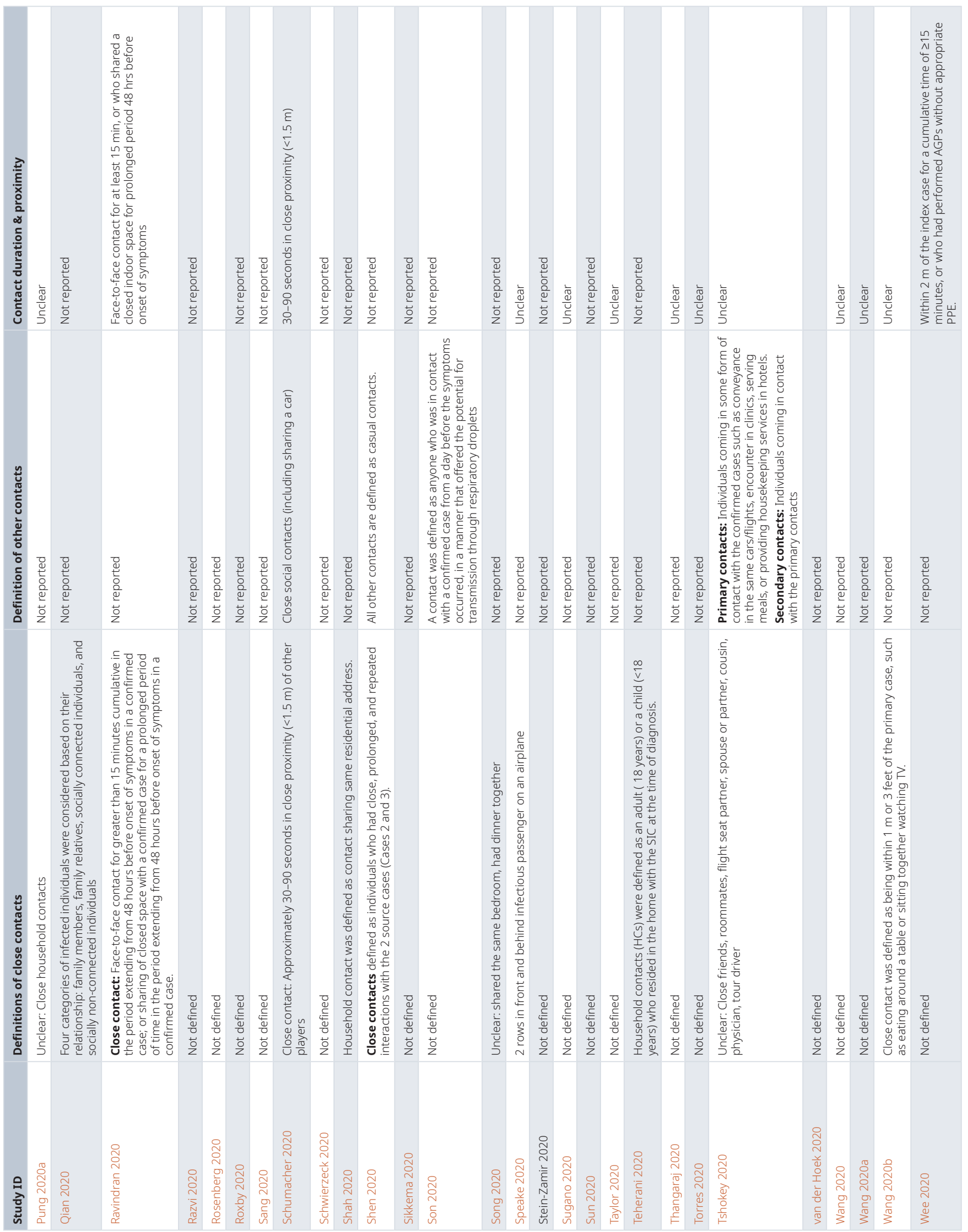




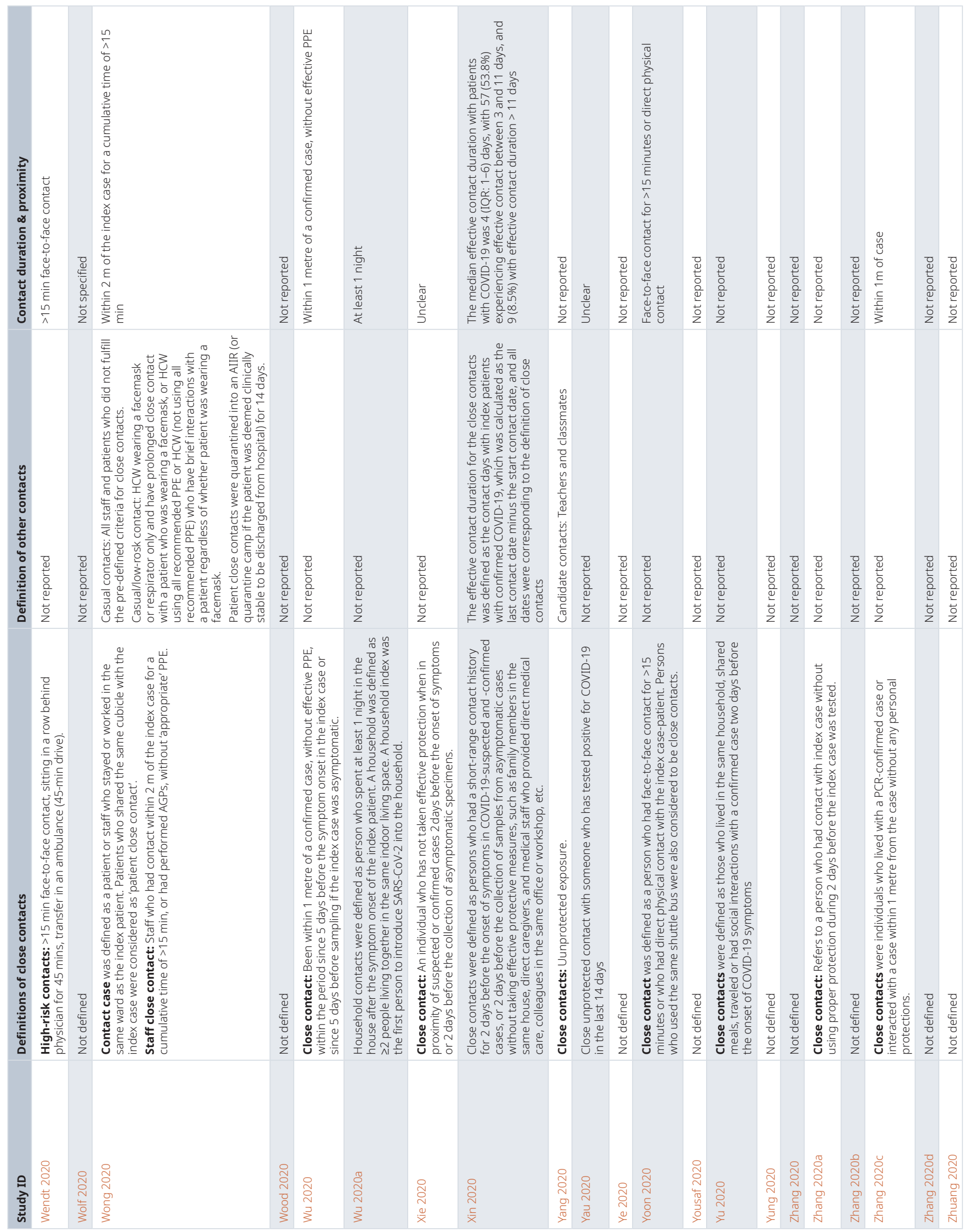




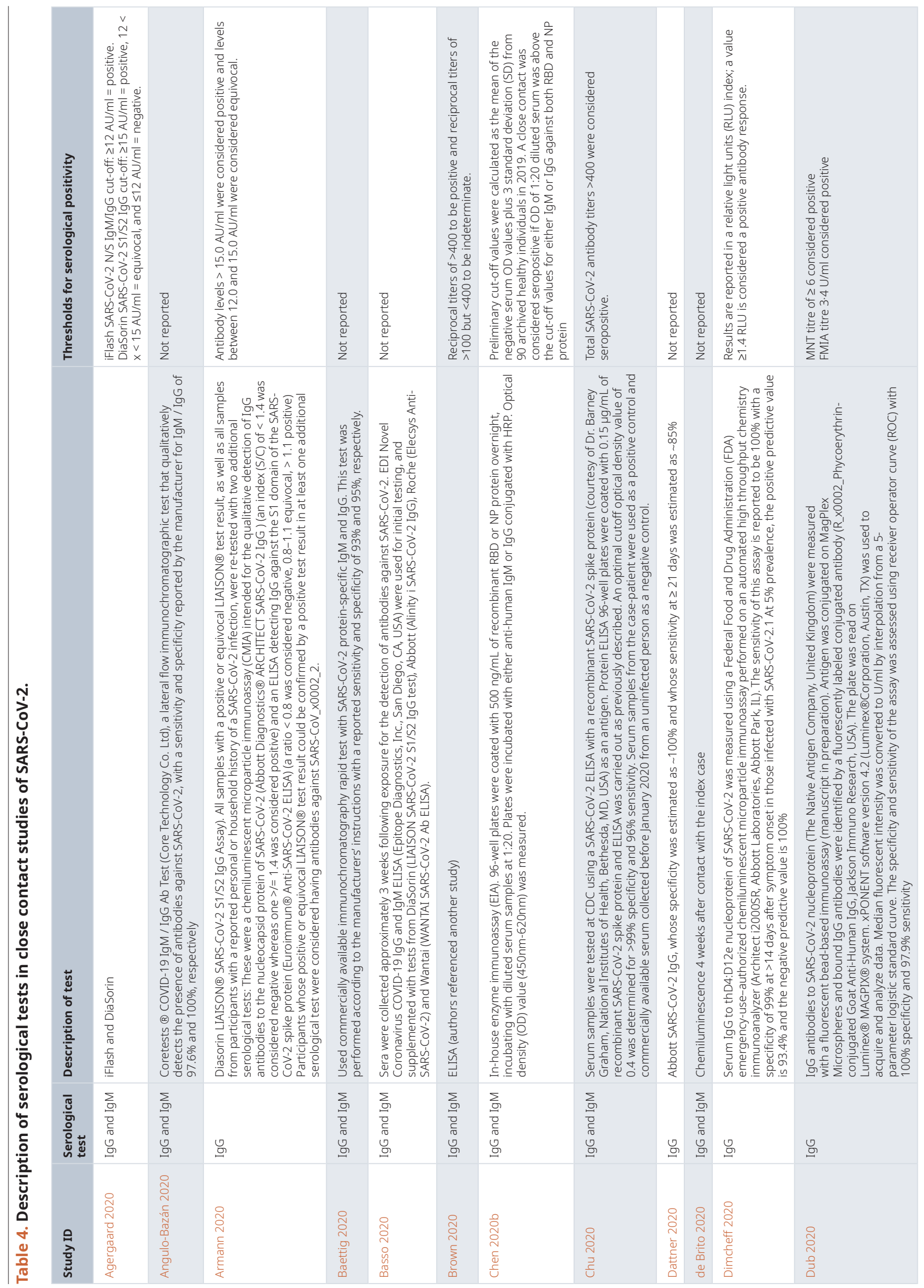




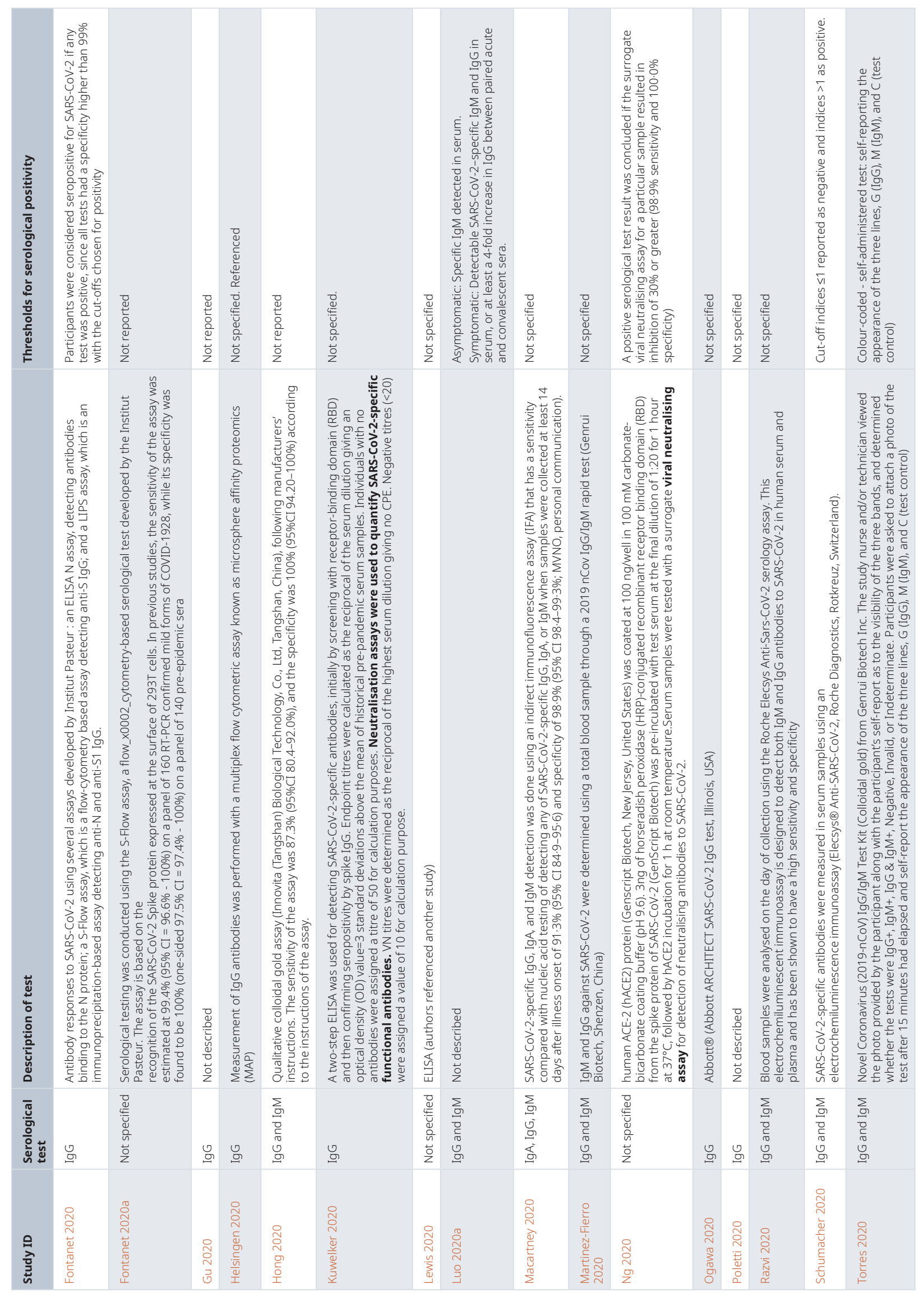


F1000Research 2021, 10:280 Last updated: 06 FEB 2023

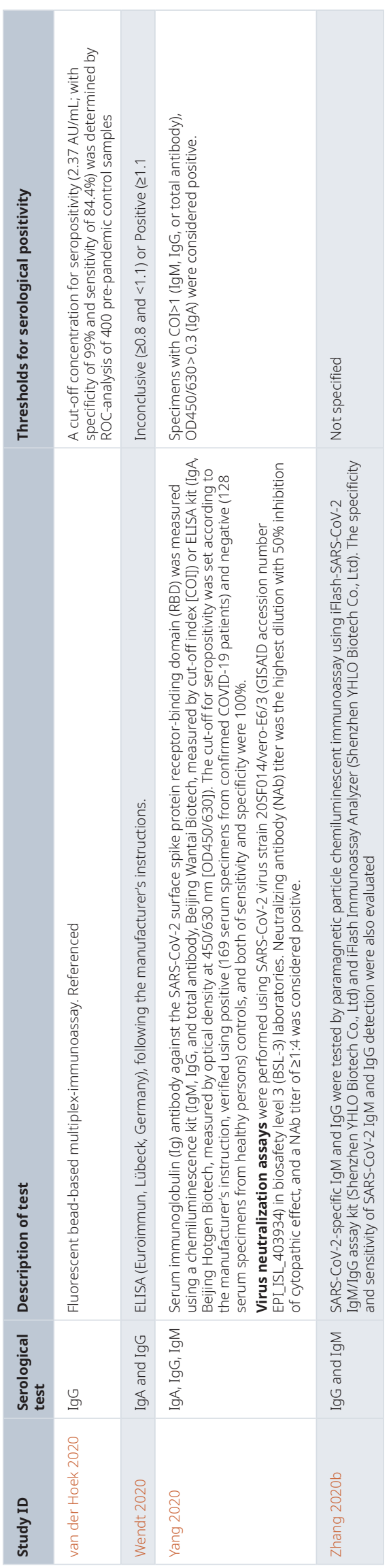


Table 5. Quality of included studies.

\begin{tabular}{|c|c|c|c|c|c|c|}
\hline Study & $\begin{array}{l}\text { Description of methods } \\
\text { and sufficient detail to } \\
\text { replicate }\end{array}$ & $\begin{array}{l}\text { Sample } \\
\text { sources } \\
\text { clear }\end{array}$ & $\begin{array}{l}\text { Analysis \& } \\
\text { reporting } \\
\text { appropriate }\end{array}$ & $\begin{array}{l}\text { Is bias } \\
\text { dealt } \\
\text { with }\end{array}$ & Applicability & Notes \\
\hline Abdulrahman 2020 & Unclear & Yes & Yes & No & Yes & \\
\hline Adamik 2020 & Unclear & Unclear & Yes & No & Unclear & \\
\hline Agergaard 2020 & No & Yes & Yes & No & Yes & \\
\hline Angulo-Bazán 2020 & Yes & No & Yes & Unclear & Yes & \\
\hline Armann 2020 & Unclear & Yes & Yes & No & Yes & \\
\hline Arnedo-Pena 2020 & Yes & Yes & Yes & Unclear & Yes & \\
\hline Baker 2020 & Unclear & Yes & Yes & Unclear & Yes & \\
\hline Baettig 2020 & Unclear & Yes & Yes & Unclear & Yes & \\
\hline Bao 2020 & Unclear & Yes & Yes & No & Yes & \\
\hline Basso 2020 & Unclear & Yes & Yes & Unclear & Yes & \\
\hline Bays 2020 & Unclear & Yes & Yes & No & Yes & \\
\hline Bi 2020 & Yes & Yes & Yes & Unclear & Yes & \\
\hline Blaisdell 2020 & Yes & No & Yes & Unclear & Yes & \\
\hline Böhmer 2020 & Yes & Yes & Yes & Unclear & Yes & \\
\hline Boscolo-Rizzo 2020 & Unclear & Yes & Yes & No & Yes & \\
\hline Brown 2020 & Yes & Yes & Yes & Unclear & Unclear & \\
\hline Burke 2020 & Unclear & No & Yes & No & Yes & \\
\hline Canova 2020 & Unclear & Yes & Yes & Unclear & Yes & \\
\hline Cariani 2020 & Unclear & Yes & Unclear & Unclear & Yes & \\
\hline Charlotte 2020 & Unclear & Yes & Yes & Unclear & Yes & \\
\hline Chaw 2020 & Unclear & Yes & Yes & Unclear & Yes & \\
\hline Chen 2020 & Unclear & Unclear & Yes & No & Unclear & \\
\hline Chen 2020a & Unclear & Yes & Yes & Unclear & Yes & \\
\hline Chen 2020b & Yes & Yes & Yes & Unclear & Yes & \\
\hline Chen 2020c & Unclear & No & Yes & No & Yes & \\
\hline Cheng 2020 & Yes & No & Yes & Unclear & Yes & \\
\hline Chu 2020 & Yes & Yes & Yes & Unclear & Yes & \\
\hline Chu 2020a & Unclear & Unclear & Unclear & No & Yes & \\
\hline Contejean 2020 & Unclear & Yes & Yes & Unclear & Yes & \\
\hline $\begin{array}{l}\text { COVID-19 National } \\
\text { Emergency Response } \\
\text { Center } 2020\end{array}$ & Unclear & No & Yes & No & Yes & \\
\hline Danis 2020 & Yes & Yes & Yes & No & Yes & \\
\hline Dattner 2020 & Yes & Yes & Yes & Unclear & Yes & \\
\hline de Brito 2020 & Yes & Yes & Unclear & Unclear & Yes & \\
\hline Deng 2020 & Unclear & No & Unclear & Unclear & Unclear & \\
\hline Desmet 2020 & Yes & Yes & Yes & No & Unclear & \\
\hline Dimcheff 2020 & Yes & Unclear & Yes & Unclear & Unclear & \\
\hline
\end{tabular}




\begin{tabular}{|c|c|c|c|c|c|c|}
\hline Study & $\begin{array}{l}\text { Description of methods } \\
\text { and sufficient detail to } \\
\text { replicate }\end{array}$ & $\begin{array}{l}\text { Sample } \\
\text { sources } \\
\text { clear }\end{array}$ & $\begin{array}{l}\text { Analysis \& } \\
\text { reporting } \\
\text { appropriate }\end{array}$ & $\begin{array}{l}\text { Is bias } \\
\text { dealt } \\
\text { with }\end{array}$ & Applicability & Notes \\
\hline Dong 2020 & Unclear & No & Unclear & No & Yes & \\
\hline Doung-ngern 2020 & Yes & Yes & Yes & Unclear & Yes & \\
\hline Draper 2020 & Yes & Yes & Yes & No & Yes & \\
\hline Dub 2020 & Yes & Yes & Yes & Unclear & Yes & \\
\hline Expert Taskforce 2020 & Unclear & Unclear & Yes & Unclear & Unclear & \\
\hline Fateh-Moghadam 2020 & Unclear & No & Yes & No & Yes & \\
\hline Firestone 2020 & Unclear & Unclear & Yes & Unclear & Yes & \\
\hline Fontanet 2020 & Yes & Yes & Yes & No & Yes & \\
\hline Fontanet 2020a & Yes & Yes & Yes & No & Yes & \\
\hline Gan 2020 & Unclear & Unclear & Unclear & Unclear & Unclear & \\
\hline Ghinai 2020 & Unclear & Unclear & Unclear & Unclear & Unclear & \\
\hline Gong 2020 & Yes & Yes & Unclear & Unclear & Unclear & \\
\hline Gu 2020 & Unclear & Unclear & Unclear & No & Unclear & \\
\hline Hamner 2020 & Unclear & Unclear & Yes & No & Yes & \\
\hline Han 2020 & Yes & Yes & Yes & Unclear & Yes & \\
\hline Heavey 2020 & Unclear & No & Yes & No & Yes & \\
\hline Helsingen 2020 & Yes & Yes & Yes & Yes & Yes & \\
\hline Hendrix 2020 & Yes & Yes & Yes & No & Yes & \\
\hline Hirschman 2020 & Unclear & Unclear & Unclear & No & Yes & \\
\hline Hobbs 2020 & Yes & Yes & Yes & Unclear & Yes & \\
\hline Hoehl 2020 & Yes & Yes & Yes & Unclear & Yes & \\
\hline Hong 2020 & Yes & Yes & Yes & Unclear & Yes & \\
\hline Hu 2020 & Unclear & No & Yes & No & Yes & \\
\hline Hua 2020 & Yes & Unclear & Yes & Unclear & Yes & \\
\hline Huang 2020 & Unclear & Unclear & Yes & No & Unclear & \\
\hline Huang 2020a & Unclear & Unclear & Yes & Unclear & Unclear & \\
\hline Islam 2020 & Yes & No & Yes & No & Yes & \\
\hline Jia 2020 & Unclear & Unclear & Yes & No & Unclear & \\
\hline Jiang 2020 & Yes & Yes & Unclear & No & Yes & \\
\hline Jing 2020 & Yes & Yes & Yes & Unclear & Yes & \\
\hline Jing 2020a & Unclear & Yes & Unclear & Unclear & Unclear & \\
\hline Jones 2020 & Unclear & Yes & Yes & Unclear & Unclear & \\
\hline Kang 2020 & Unclear & Unclear & Unclear & Unclear & Unclear & \\
\hline Kant 2020 & Unclear & Yes & Unclear & No & Unclear & \\
\hline Kawasuji 2020 & Unclear & Yes & Unclear & Unclear & Unclear & \\
\hline Khanh 2020 & Yes & Yes & Yes & No & Yes & \\
\hline Kim 2020 & Unclear & Yes & Yes & Unclear & Yes & \\
\hline Kim 2020a & Unclear & Yes & Yes & No & Unclear & \\
\hline
\end{tabular}




\begin{tabular}{|c|c|c|c|c|c|c|}
\hline Study & $\begin{array}{l}\text { Description of methods } \\
\text { and sufficient detail to } \\
\text { replicate }\end{array}$ & $\begin{array}{l}\text { Sample } \\
\text { sources } \\
\text { clear }\end{array}$ & $\begin{array}{l}\text { Analysis \& } \\
\text { reporting } \\
\text { appropriate }\end{array}$ & $\begin{array}{l}\text { Is bias } \\
\text { dealt } \\
\text { with }\end{array}$ & Applicability & Notes \\
\hline Kim 2020b & Yes & Yes & Yes & No & Yes & \\
\hline Kumar 2020 & Unclear & Yes & Unclear & No & Unclear & \\
\hline Kuwelker 2020 & Unclear & Yes & Yes & Unclear & Yes & \\
\hline Kwok 2020 & Unclear & Unclear & Yes & Unclear & Unclear & \\
\hline Ladhani 2020 & No & Unclear & Unclear & No & Yes & \\
\hline Ladhani 2020a & Unclear & Unclear & Yes & Unclear & Yes & \\
\hline Laws 2020 & Unclear & Unclear & Yes & Unclear & Yes & \\
\hline Laxminarayan 2020 & Yes & No & Yes & No & Yes & \\
\hline Lee 2020 & Unclear & Unclear & Yes & Unclear & Unclear & \\
\hline Lee 2020a & Unclear & No & Yes & No & Yes & \\
\hline Lewis 2020 & Yes & Yes & Yes & No & Yes & \\
\hline Li 2020 & Unclear & Yes & Unclear & No & Unclear & \\
\hline Li 2020a & Unclear & Unclear & Unclear & Unclear & Unclear & \\
\hline Li 2020b & Unclear & Yes & Unclear & Unclear & Unclear & \\
\hline Li 2020c & Unclear & No & Unclear & Unclear & Unclear & \\
\hline Li 2020d & Yes & Yes & Yes & No & Yes & \\
\hline Liu 2020 & Unclear & Unclear & Unclear & No & Yes & \\
\hline Liu 2020a & Yes & Yes & Yes & Unclear & Unclear & \\
\hline Liu 2020b & Unclear & Yes & Yes & Unclear & Yes & \\
\hline Liu 2020c & Unclear & Unclear & Unclear & No & Unclear & \\
\hline López 2020 & Unclear & Unclear & Yes & Unclear & Yes & \\
\hline Lopez Bernal 2020 & Yes & Unclear & Yes & No & Yes & \\
\hline Lucey 2020 & Unclear & Yes & Yes & No & Yes & \\
\hline Luo 2020 & Unclear & Yes & Yes & Unclear & Yes & \\
\hline Luo 2020a & Unclear & Yes & Yes & Yes & Yes & $\begin{array}{l}\text { They use multiple } \\
\text { imputation to minimise } \\
\text { inferential bias, and } \\
\text { they discuss recall } \\
\text { bias, selection bias and } \\
\text { regression to the mean. }\end{array}$ \\
\hline Lyngse 2020 & Yes & Unclear & Yes & Yes & Yes & $\begin{array}{l}\text { They investigate bias } \\
\text { within their data and } \\
\text { discuss this fairly fully }\end{array}$ \\
\hline Ma 2020 & Unclear & Unclear & Unclear & Unclear & Unclear & \\
\hline Macartney 2020 & Yes & Unclear & Yes & Unclear & Yes & \\
\hline Malheiro 2020 & Yes & Unclear & Yes & Unclear & Yes & \\
\hline Maltezou 2020 & Unclear & Unclear & Unclear & Unclear & Yes & \\
\hline Maltezou 2020a & Unclear & Unclear & Unclear & No & Yes & \\
\hline Mao 2020 & Unclear & Unclear & Yes & No & Unclear & \\
\hline Martinez-Fierro 2020 & Unclear & Yes & Yes & No & Yes & \\
\hline
\end{tabular}




\begin{tabular}{|c|c|c|c|c|c|c|}
\hline Study & $\begin{array}{l}\text { Description of methods } \\
\text { and sufficient detail to } \\
\text { replicate }\end{array}$ & $\begin{array}{l}\text { Sample } \\
\text { sources } \\
\text { clear }\end{array}$ & $\begin{array}{l}\text { Analysis \& } \\
\text { reporting } \\
\text { appropriate }\end{array}$ & $\begin{array}{l}\text { Is bias } \\
\text { dealt } \\
\text { with }\end{array}$ & Applicability & Notes \\
\hline Mponponsuo 2020 & Unclear & Yes & Yes & Yes & Yes & $\begin{array}{l}\text { Recall bias was minimized } \\
\text { by examining multiple } \\
\text { data sources for both } \\
\text { index cases and exposed } \\
\text { persons }\end{array}$ \\
\hline Ng 2020 & Unclear & Yes & Yes & Yes & Yes & $\begin{array}{l}\text { Authors looked at } \\
\text { differences that could } \\
\text { have led to bias }\end{array}$ \\
\hline Ning 2020 & Unclear & Unclear & Unclear & Unclear & Unclear & \\
\hline Njuguna_2020 & Unclear & Unclear & Yes & Unclear & Yes & \\
\hline Ogawa 2020 & Unclear & Unclear & Yes & No & Yes & \\
\hline Paireau 2020 & Unclear & Yes & Yes & Unclear & Yes & \\
\hline Park 2020 & Unclear & Yes & Yes & Unclear & Yes & \\
\hline Park 2020a & Unclear & No & Yes & No & Yes & \\
\hline Park 2020b & Unclear & Yes & Yes & No & Unclear & \\
\hline Passarelli 2020 & Unclear & No & Unclear & Unclear & Yes & \\
\hline Patel 2020 & Yes & Yes & Yes & Unclear & Unclear & \\
\hline Pavli 2020 & Unclear & Yes & Yes & No & Yes & \\
\hline Phiriyasart 2020 & Yes & Yes & Yes & No & Yes & \\
\hline Poletti 2020 & Unclear & Yes & Yes & Yes & Unclear & \\
\hline Pung 2020 & Yes & Unclear & Yes & Unclear & Yes & \\
\hline Pung 2020a & Unclear & No & Unclear & Unclear & Unclear & \\
\hline Qian 2020 & Unclear & Unclear & Unclear & No & Unclear & \\
\hline Ravindran 2020 & Unclear & Unclear & Unclear & Unclear & Unclear & \\
\hline Razvi 2020 & Unclear & Yes & Yes & No & Yes & \\
\hline Rosenberg 2020 & Yes & Yes & Yes & No & Yes & \\
\hline Roxby 2020 & Yes & Yes & Yes & Unclear & Yes & \\
\hline Sang 2020 & Unclear & Yes & Unclear & No & Unclear & \\
\hline Schumacher 2020 & Unclear & Yes & Unclear & Unclear & Yes & \\
\hline Schwierzeck 2020 & Unclear & Yes & Yes & Unclear & Yes & \\
\hline Shah 2020 & Unclear & No & Unclear & No & Yes & \\
\hline Shen 2020 & Yes & Yes & Yes & Unclear & Yes & \\
\hline Sikkema 2020 & Unclear & Yes & Yes & Unclear & Yes & \\
\hline Son 2020 & Unclear & Unclear & Yes & No & Yes & \\
\hline Song 2020 & Unclear & Yes & Yes & Unclear & Yes & \\
\hline Speake 2020 & Unclear & Yes & Yes & Unclear & Yes & \\
\hline Sugano 2020 & Unclear & Unclear & Yes & Unclear & Yes & \\
\hline Stein-Zamir 2020 & Yes & Unclear & Yes & No & Yes & \\
\hline Sun 2020 & Unclear & Unclear & Unclear & Unclear & Unclear & \\
\hline Taylor 2020 & Yes & Yes & Yes & Unclear & Yes & \\
\hline Teherani 2020 & Unclear & Yes & Yes & Unclear & Yes & \\
\hline
\end{tabular}




\begin{tabular}{|c|c|c|c|c|c|c|}
\hline Study & $\begin{array}{l}\text { Description of methods } \\
\text { and sufficient detail to } \\
\text { replicate }\end{array}$ & $\begin{array}{l}\text { Sample } \\
\text { sources } \\
\text { clear }\end{array}$ & $\begin{array}{l}\text { Analysis \& } \\
\text { reporting } \\
\text { appropriate }\end{array}$ & $\begin{array}{l}\text { Is bias } \\
\text { dealt } \\
\text { with }\end{array}$ & Applicability & Notes \\
\hline Thangaraj 2020 & Unclear & Yes & Yes & Unclear & Unclear & \\
\hline Torres 2020 & Yes & Unclear & Yes & Unclear & Yes & \\
\hline Tshokey 2020 & Unclear & Yes & Yes & Unclear & Yes & \\
\hline van der Hoek 2020 & Unclear & Yes & Yes & No & Yes & \\
\hline Wang 2020 & Unclear & Yes & Unclear & Unclear & Yes & \\
\hline Wang 2020a & Yes & Unclear & Yes & Unclear & Yes & \\
\hline Wang 2020b & Yes & Yes & Yes & No & Yes & \\
\hline Wee 2020 & Yes & Yes & Yes & Unclear & Yes & \\
\hline Wendt 2020 & Yes & Yes & Yes & Unclear & Yes & \\
\hline Wolf 2020 & Yes & Yes & Yes & Unclear & Yes & \\
\hline Wong 2020 & Yes & Yes & Yes & Unclear & Yes & \\
\hline Wood 2020 & Unclear & No & Yes & Unclear & Yes & \\
\hline Wu 2020 & Yes & Unclear & Yes & Unclear & Yes & \\
\hline Wu 2020a & Yes & Unclear & Yes & Unclear & Yes & \\
\hline Xie 2020 & Unclear & Yes & Yes & Unclear & Yes & \\
\hline Xin 2020 & Yes & No & Yes & No & Yes & \\
\hline Yang 2020 & Unclear & Yes & Unclear & Unclear & Yes & \\
\hline Yau 2020 & Unclear & Yes & Unclear & Unclear & Unclear & \\
\hline Ye 2020 & Unclear & Unclear & Unclear & Unclear & Unclear & \\
\hline Yoon 2020 & Yes & Yes & Yes & Unclear & Yes & \\
\hline Yousaf 2020 & Unclear & Yes & Unclear & Unclear & Unclear & \\
\hline Yu 2020 & Yes & No & Yes & No & Yes & \\
\hline Yung 2020 & Unclear & Yes & Yes & No & Yes & \\
\hline Zhang 2020 & Unclear & Unclear & Unclear & No & Unclear & \\
\hline Zhang 2020a & Yes & Unclear & Yes & Unclear & Unclear & \\
\hline Zhang 2020b & Unclear & Yes & Unclear & Unclear & Yes & \\
\hline Zhang 2020c & Unclear & Unclear & Unclear & Unclear & Unclear & \\
\hline Zhang 2020d & Unclear & Yes & Unclear & Unclear & Unclear & \\
\hline Zhuang 2020 & Unclear & No & Yes & No & Unclear & \\
\hline
\end{tabular}

in the description of the tests. Fifteen studies determined the antibody responses to SARS-CoV-2 spike proteins using Immunoglobulin $\mathrm{G}$ (IgG) and $\operatorname{IgM}$ while 11 used only IgG. In 17 studies, the threshold for serological positivity was not reported. Three studies (Kuwelker 2020, Ng 2020, Yang 2020) performed neutralisation assays to confirm positive serologic samples. In one study (Torres 2020), study participants self-administered the serological tests.

Three studies (Ladhani 2020a, Speake 2020, Yang 2020) performed viral culture, while 10 studies (Böhmer 2020, Firestone 2020,
Jiang 2020, Ladhani 2020a, Lucey 2020, Pung 2020, Sikkema 2020, Speake 2020, Taylor 2020, Wang 2020) performed genome sequencing (GS) plus phylogenetic analysis.

Frequency of SARS-CoV-2 attack rates (ARs)

Twenty-three studies reported data on attack rates using RT-PCR (Table 6). The settings included healthcare $(n=3)$, household $(n=8)$, public transport $(n=2)$, educational settings $(n=3)$. In one study of 84 children in daycare centres during the first few weeks of the pandemic (Desmet 2020), the AR was 0\%; similar results were reported in another study of hospital healthcare workers 


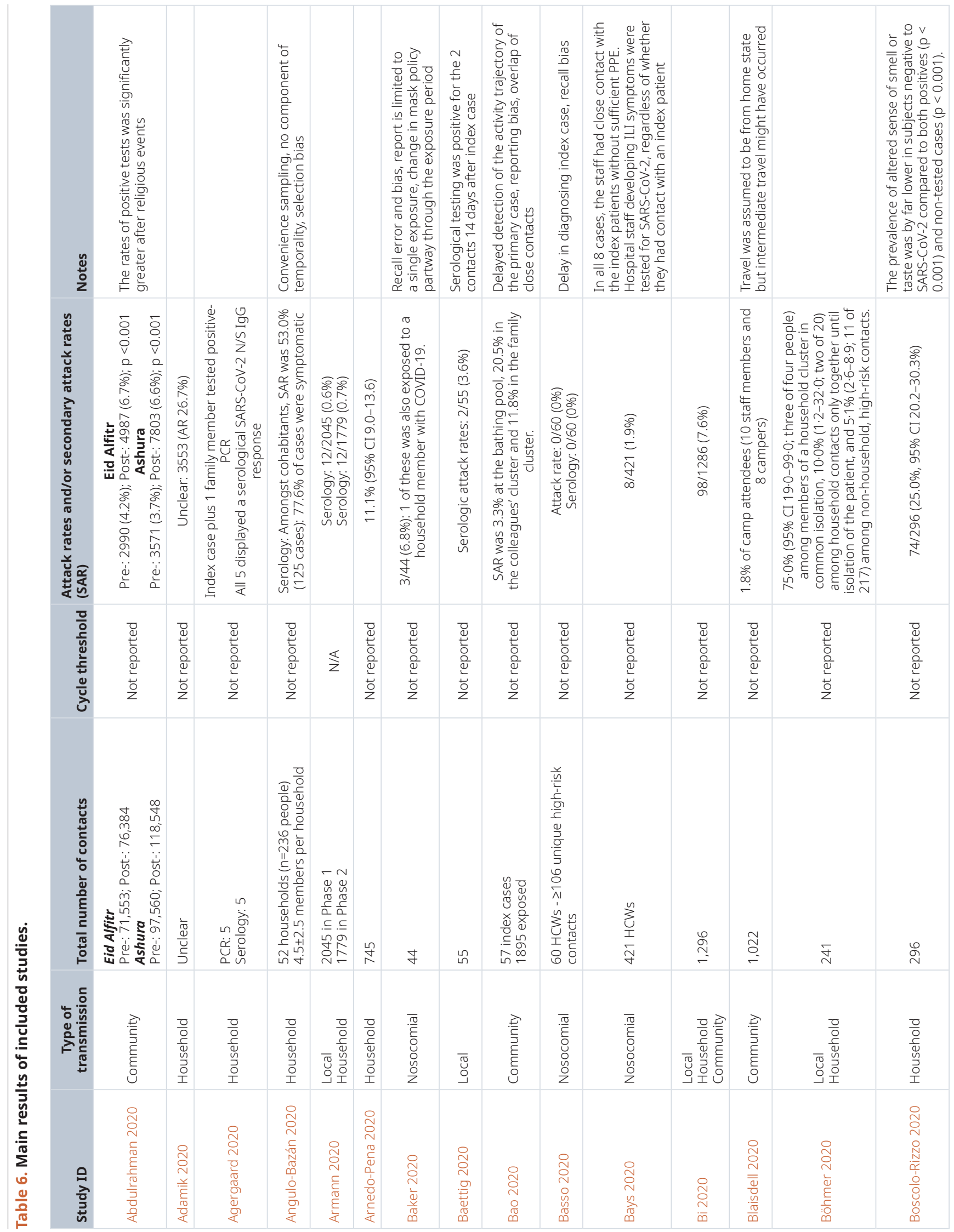




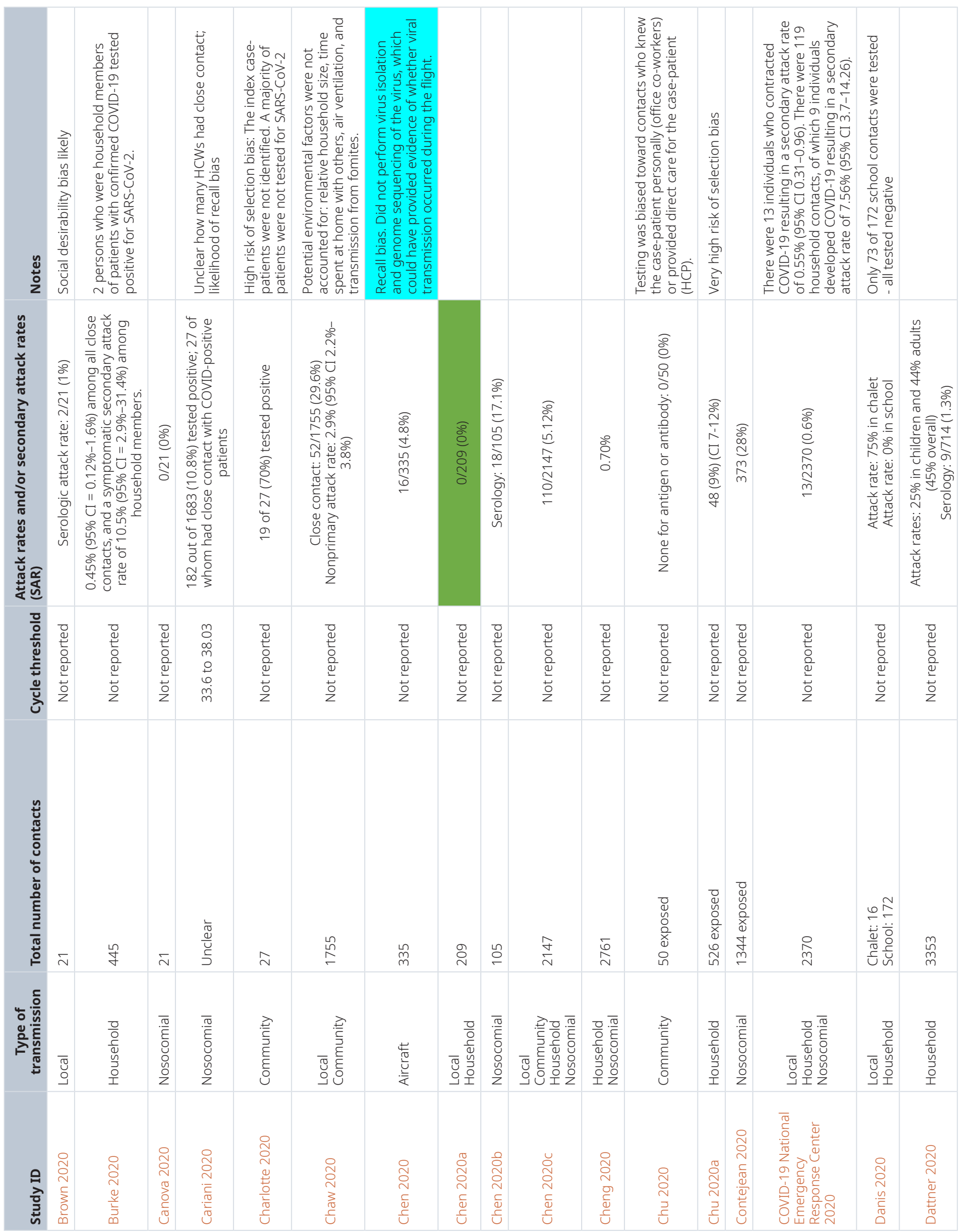




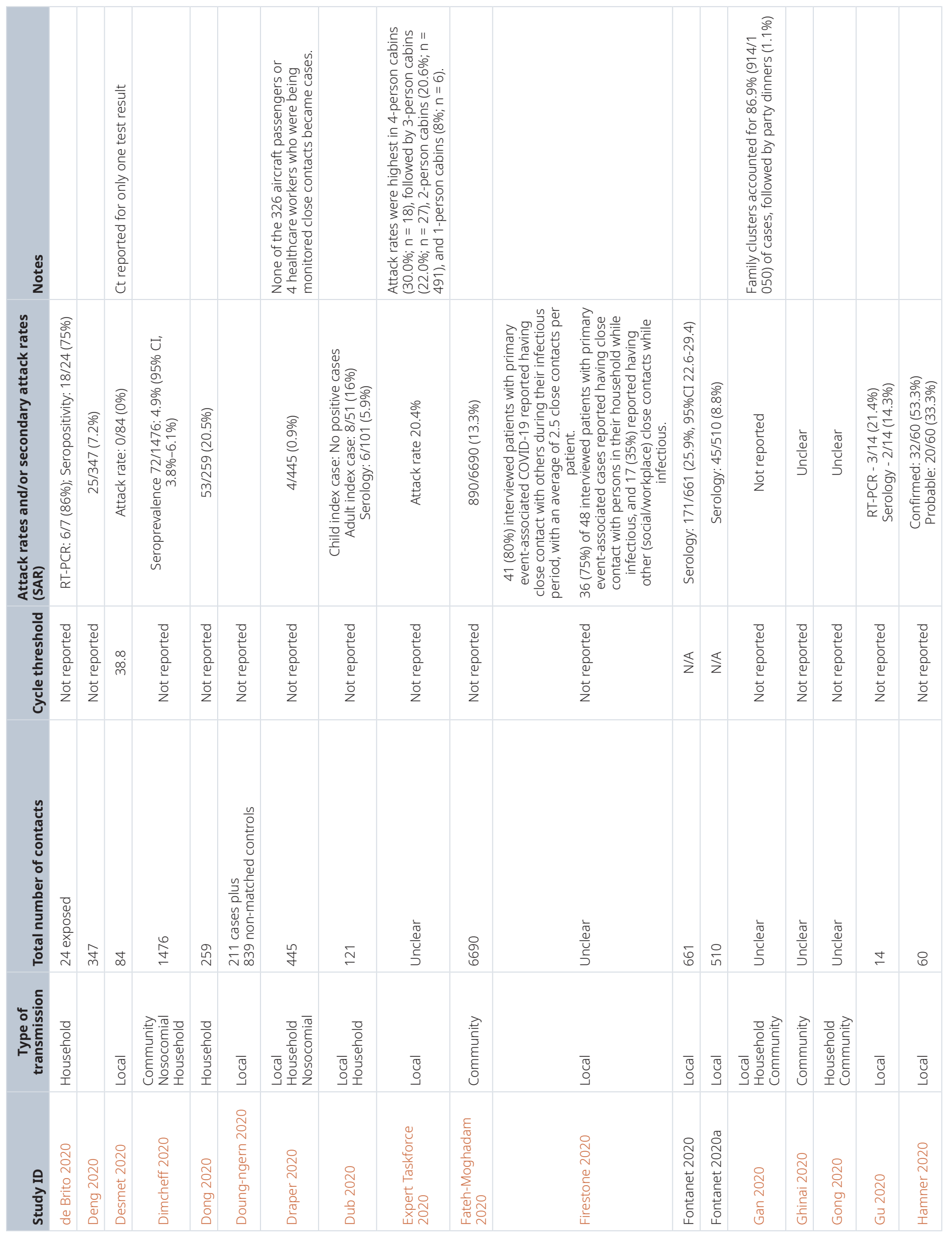




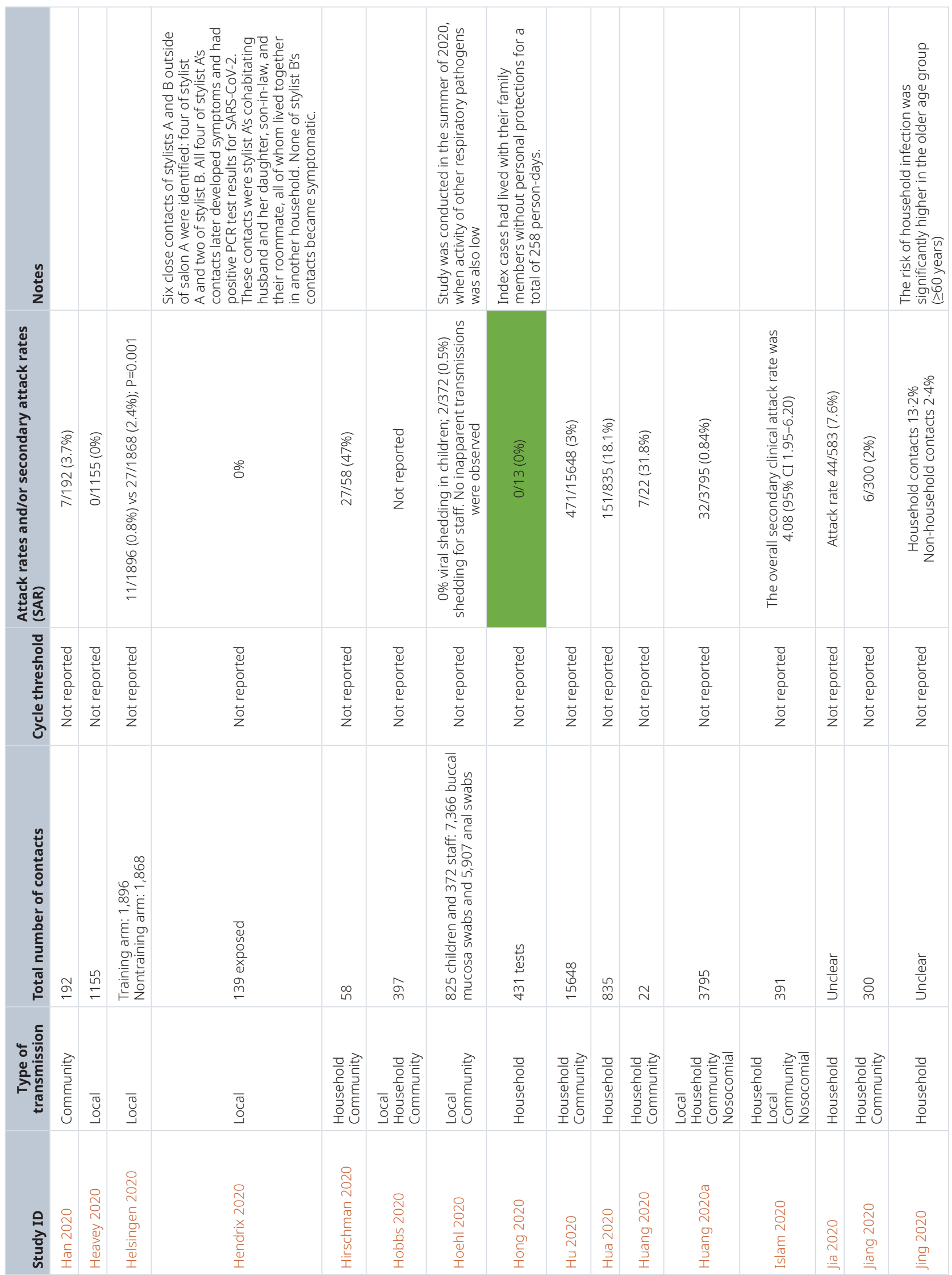




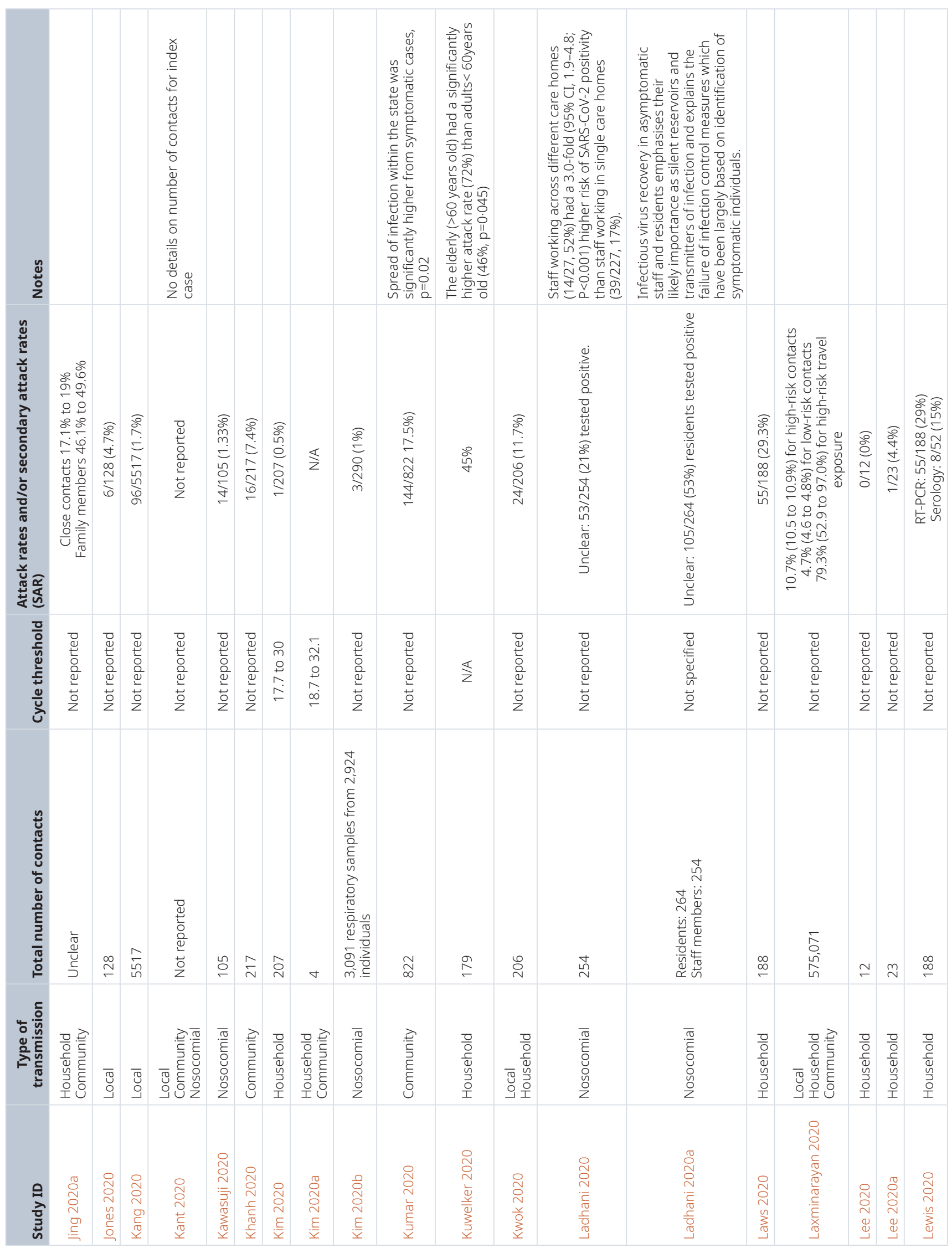




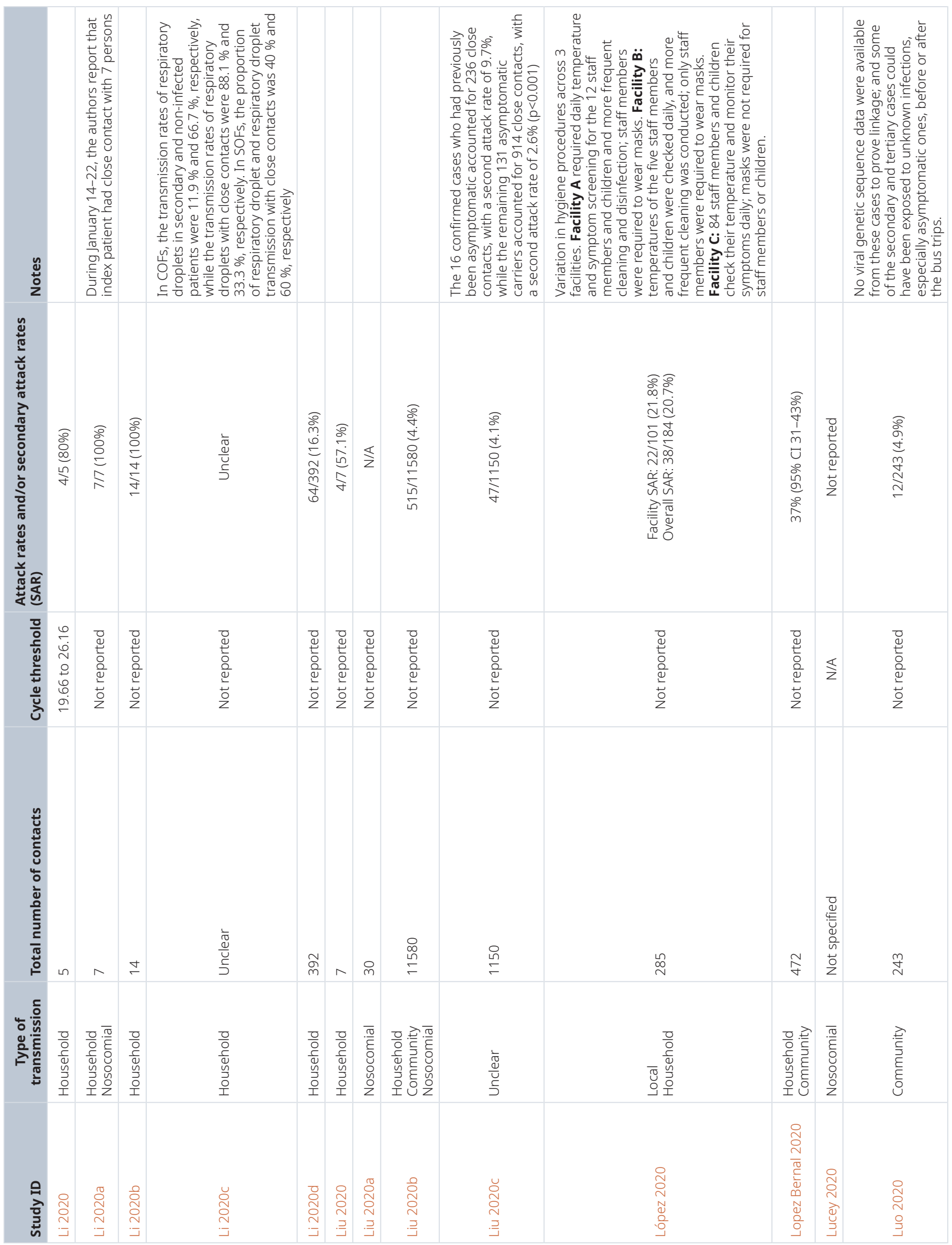




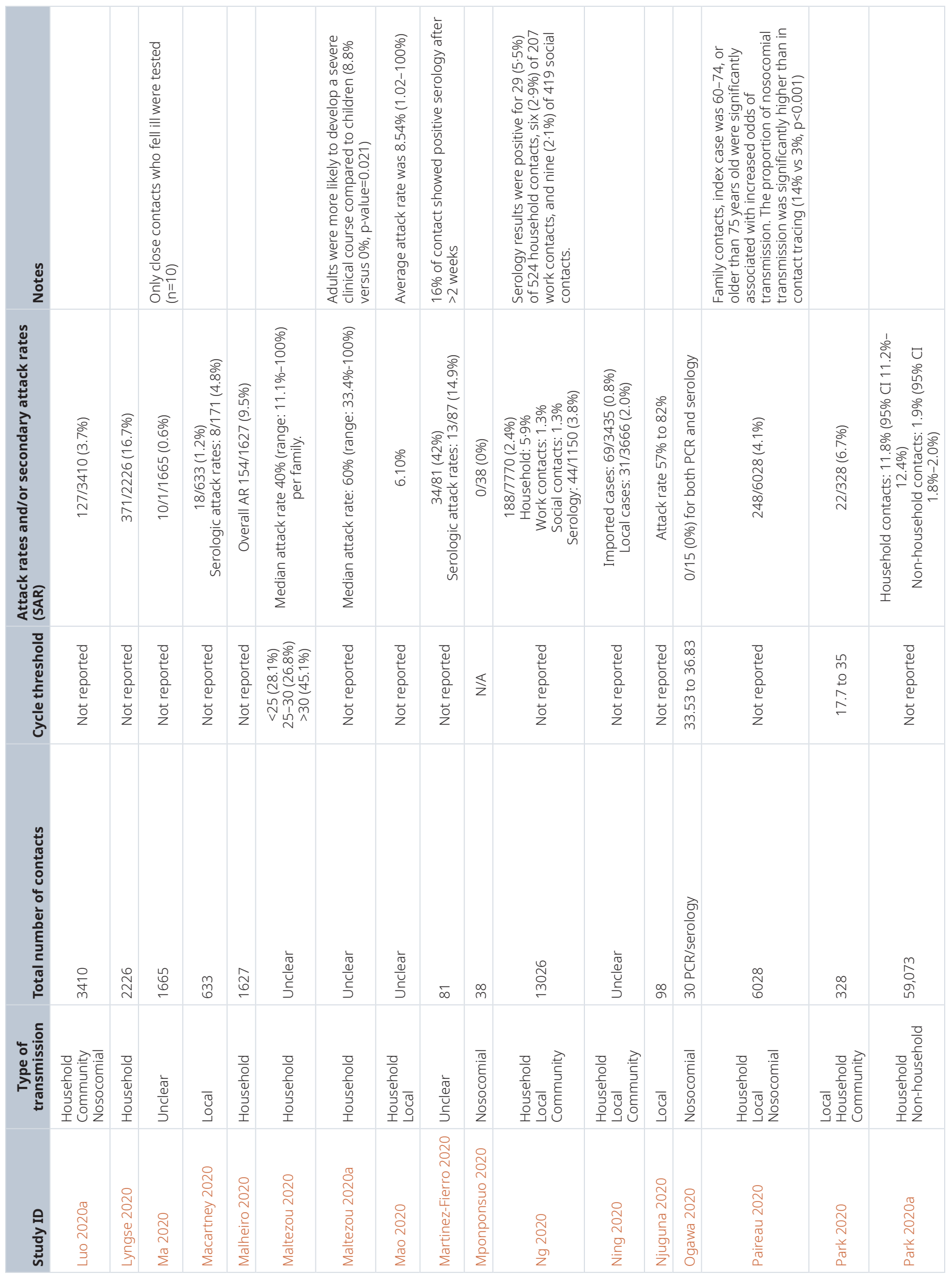




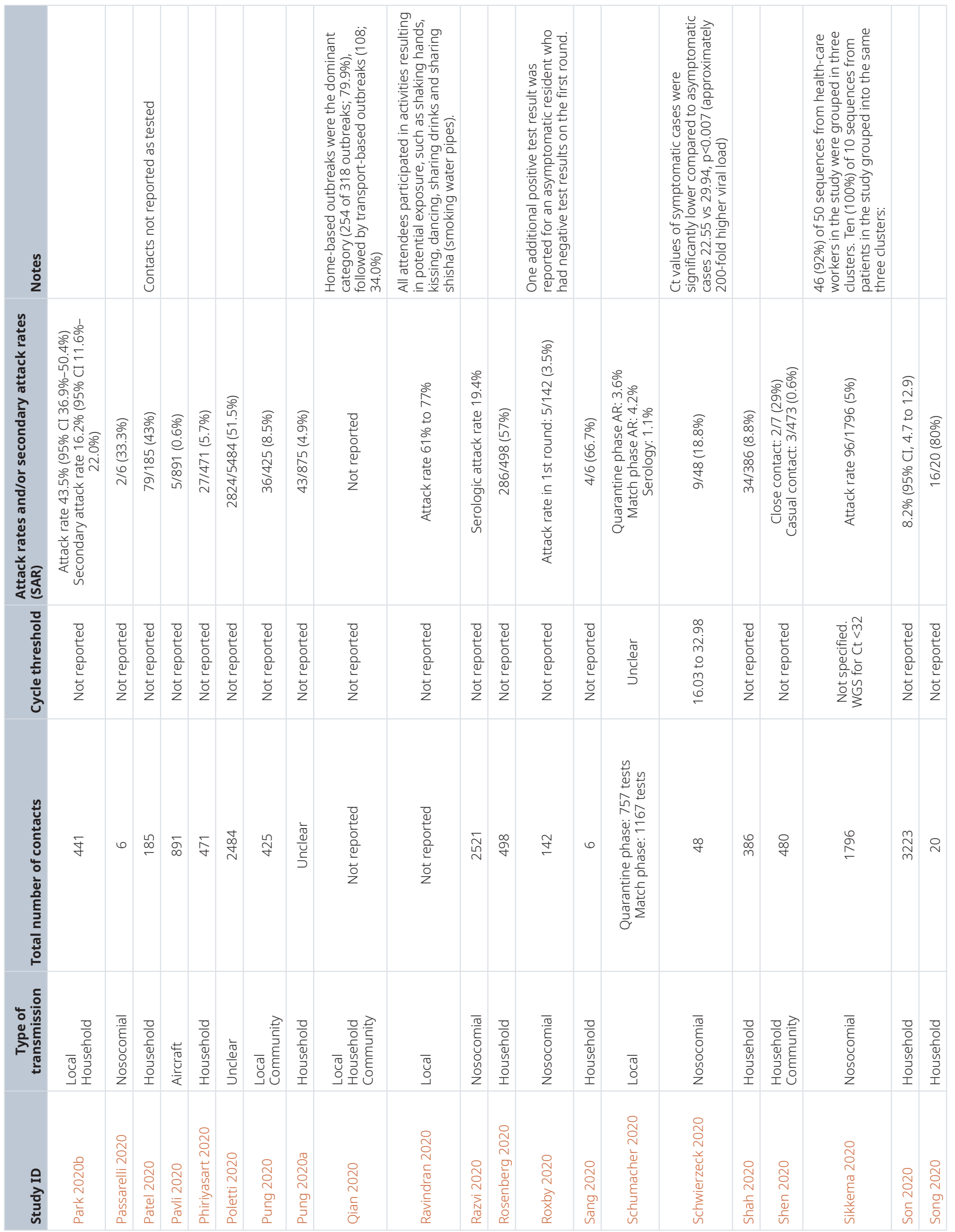




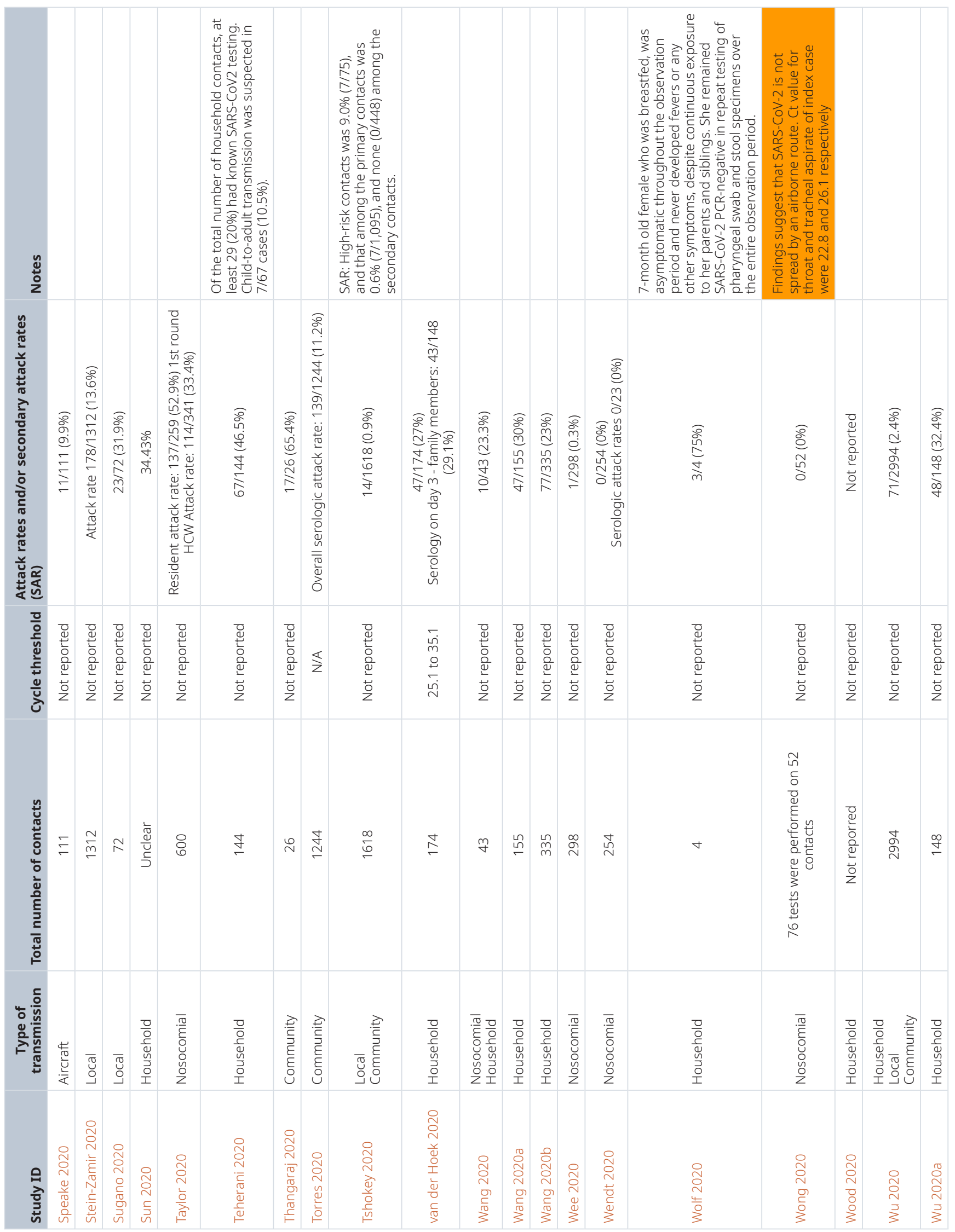




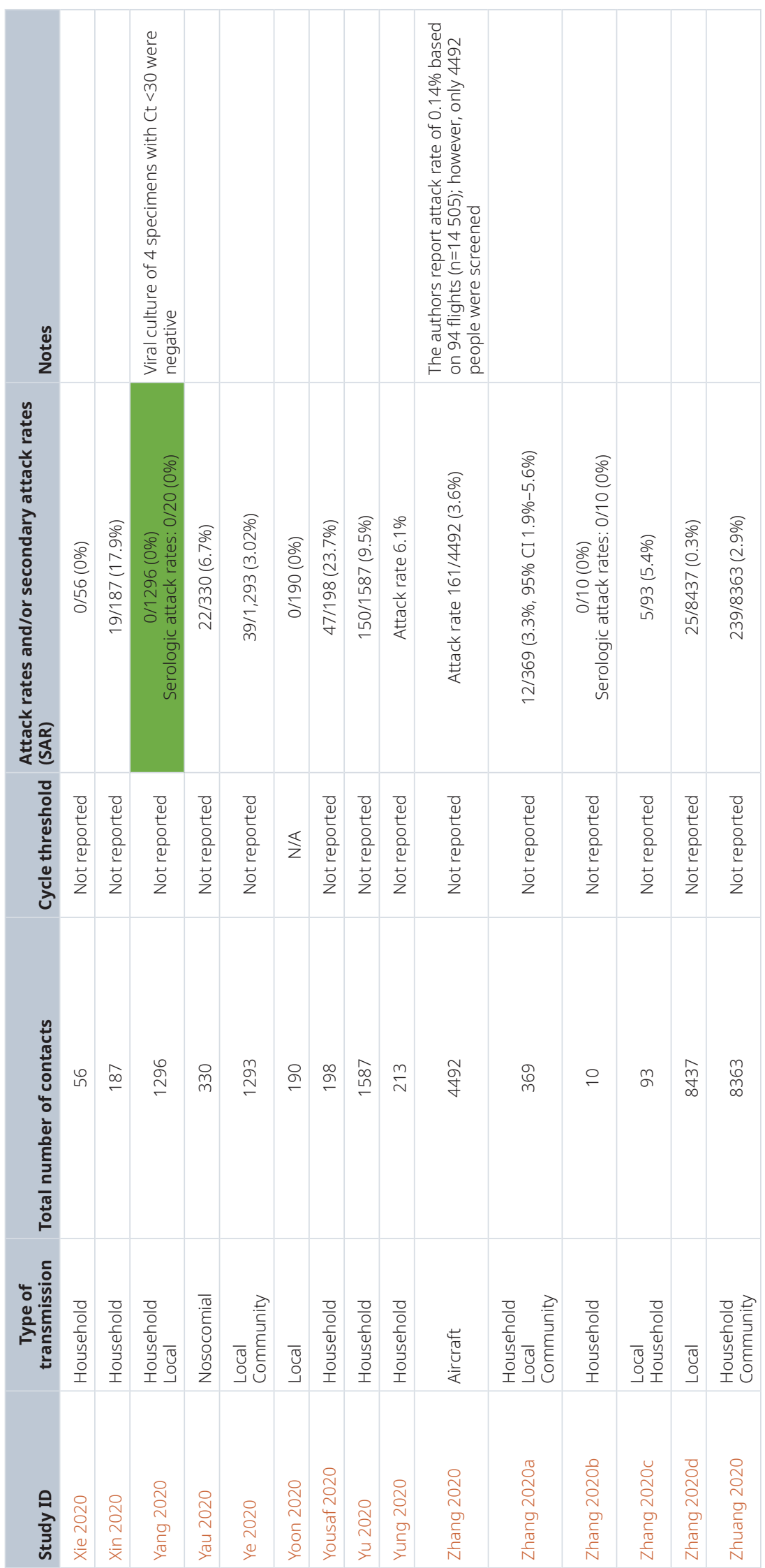


(Basso 2020). The frequency of ARs in the remaining 21 studies ranged from 3.5 to $75 \%$ (Figure 3a). The ARs were highest in weddings (69\%), prison (69.5\%) and households (75\%). Attack rates appeared lower in healthcare settings; two healthcare settings with higher ARs (Ladhani 2020, Ladhani 2020a) included nursing home residents - the definition of SARS-CoV-2 infection in both studies did not include the full constellation of respiratory and non-respiratory symptoms. In sports settings, the AR during matches was between $4.2 \%$ and $4.7 \%$.

Twenty-nine studies reported data on ARs using serology (Table 6). The settings included educational $(n=4)$, households $(n=4)$ and healthcare $(n=3)$. In eight studies, the frequency of attack was $0 \%$. The frequency of attacks in the remaining 21 studies ranged from $0.7 \%$ to $75 \%$ (Figure $3 b$ ). The frequency of attacks was highest in households but lower in educational settings especially daycare centres.

\section{Frequency of SARS-CoV-2 secondary ARs}

Overall, 126 studies (73.7\%) reported data on secondary ARs (Table 6). The studies reported the rates based on RT-PCR tests, except for one study (Angulo-Bazán 2020) that used serology. In 16 of these studies, the SAR was $0 \%$. The secondary ARs in the remaining 110 studies ranged from 0.3 to $100 \%$ (see Figure 4). The highest frequencies of secondary ARs (75-100\%) occurred in household or quarantine settings; similar findings were observed when studies with higher reporting quality were examined (57-75\%). In the three studies of index or primary cases with recurrent infections, there was no positive case amongst the 1518 close contacts across the studies.

\section{Risk of infection}

Forty-six studies (26.9\%) reported results on the risk of infection (Table 7). One study of airline passengers (Khanh 2020) showed that seating proximity was significantly associated with the risk of contracting SARS-CoV-2 (RR 7.3, 95\% CI 1.2 46.2); a second study (Speake 2020) reported that not sitting by the window was associated with a significantly increased risk of infection ( $R R$ 5.2; 95\% CI 1.6-16.4; p<0.007)). The results of five studies (Chen 2020b, Doung-ngern 2020, Hobbs 2020, Wang 2020b, Wu 2020) showed that use of face covering during close contact with infected cases was associated with significantly lower risks of infection compared with no face covering; findings from one of these studies (Doung-ngern 2020) showed that wearing masks all the time during contact was not significantly different from wearing masks sometimes. The result of one study (Rosenberg 2020) showed that the incidence of infection significantly increased with age $(\mathrm{p}<0.0001)$, while those from another study (Poletti 2020) showed that being 70 years or older was associated with a significantly increased risk of SARS-CoV-2-related death $(\mathrm{p}<0.001)$, while another study (Zhang 2020a) reported that elderly close contacts ( $\geq 60$ years) had a higher SAR compared with younger age groups. Findings from five studies (Bi 2020, Hu 2020a, Islam 2020, Luo 2020a, Wu 2020, Zhang 2020a) showed that household contact settings had significantly higher risks of infection compared with other types of contact settings, e.g., social, healthcare, workplace and public transport. One study (Lewis 2020) showed that the risk of infection was significantly increased amongst household contacts who were immunocompromised (OR 15.9, 95\% CI 2.4-106.9). Finally, three studies

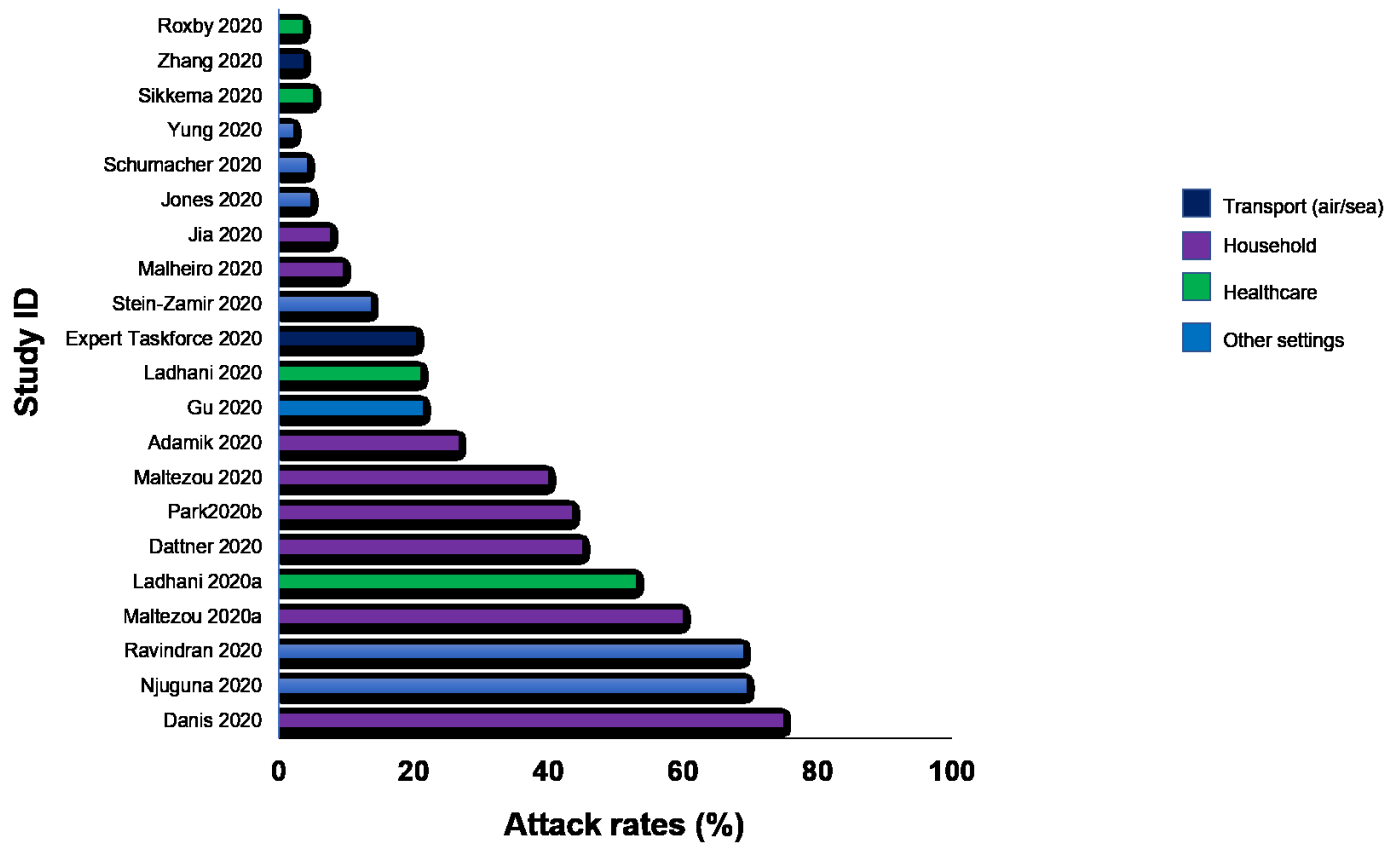

Figure 3a. Primary attack rates of SARS-CoV-2 in close contacts (PCR). 


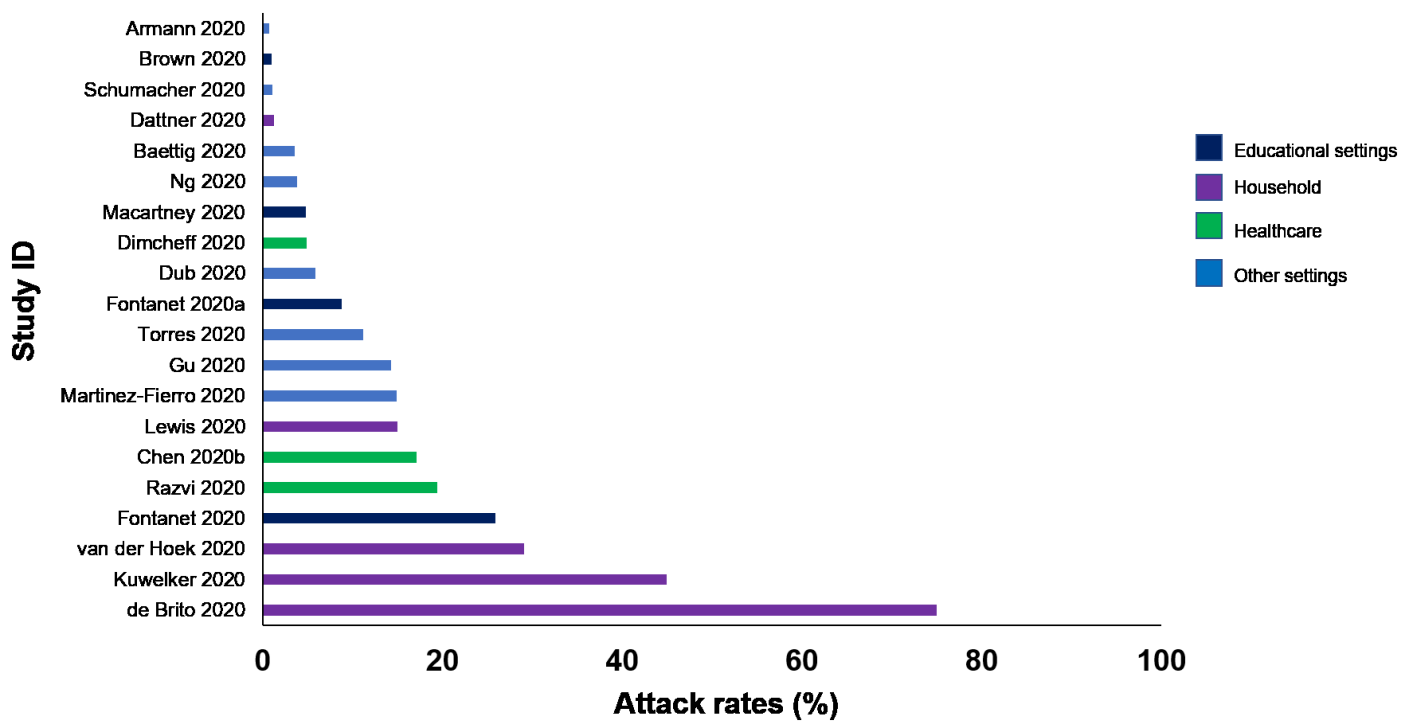

Figure 3b. Primary attack rates of SARS-CoV-2 in close contacts (serology).

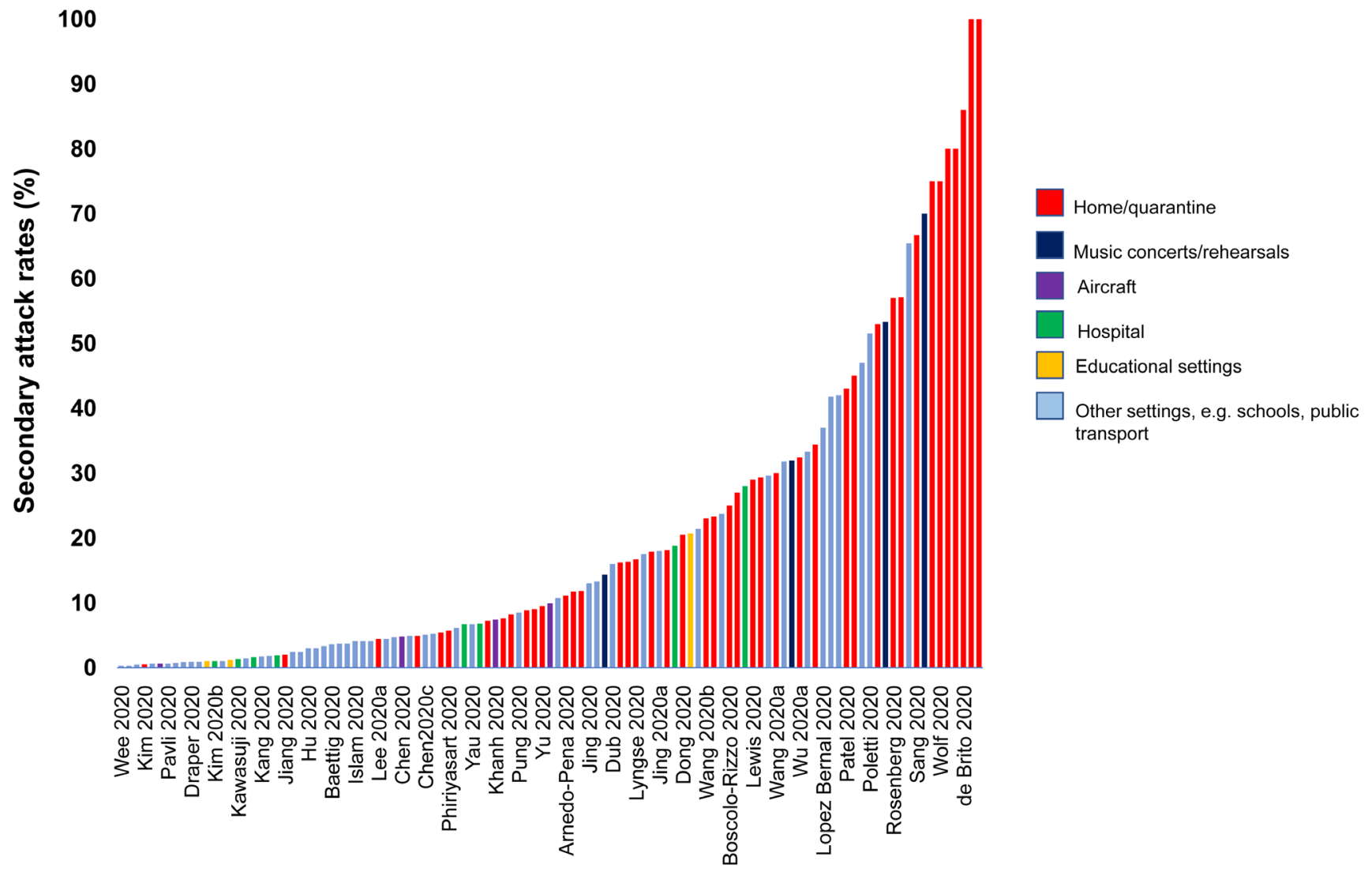

Figure 4. Frequency of secondary attack rates of SARS-CoV-2 with Close Contacts. 


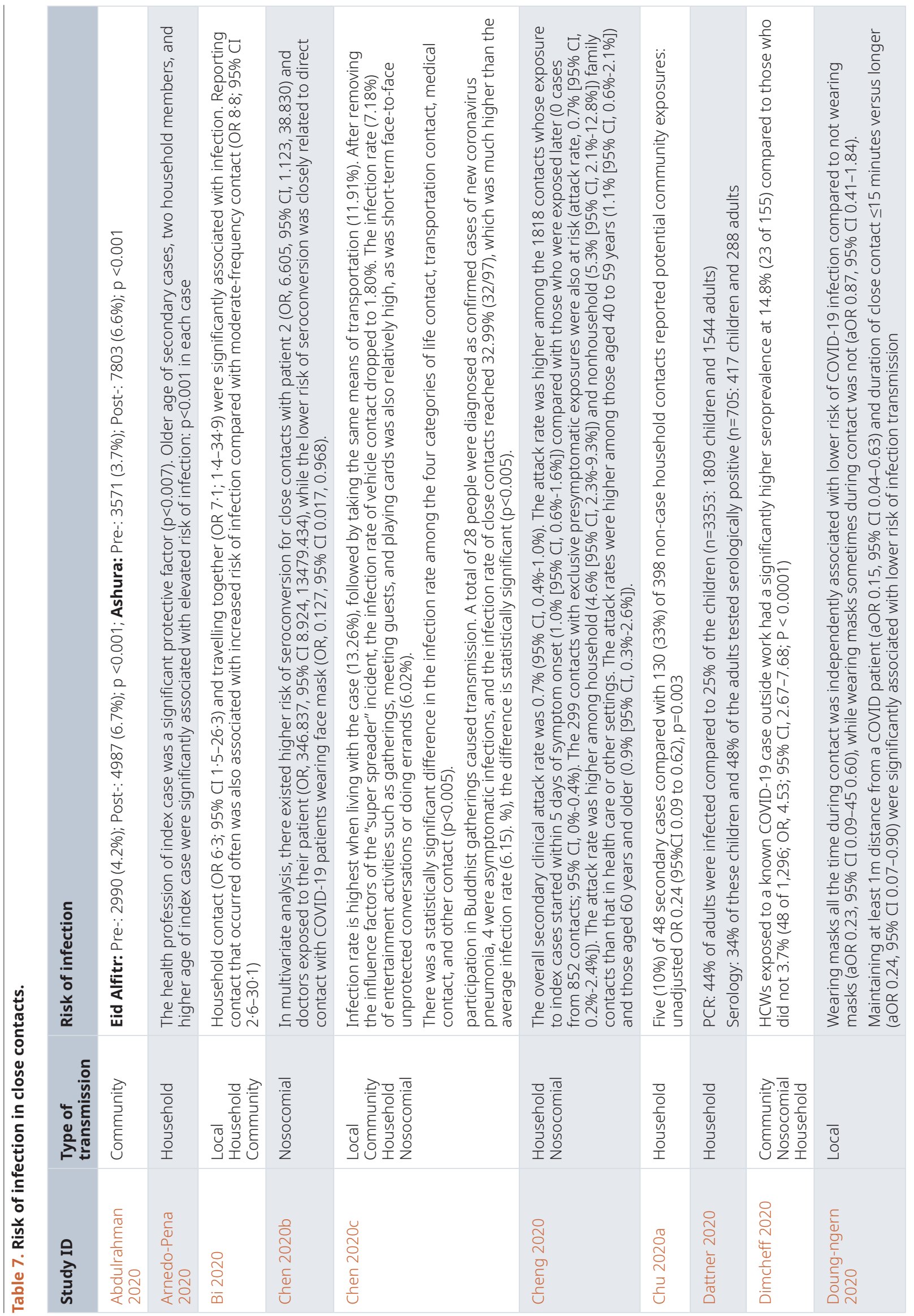




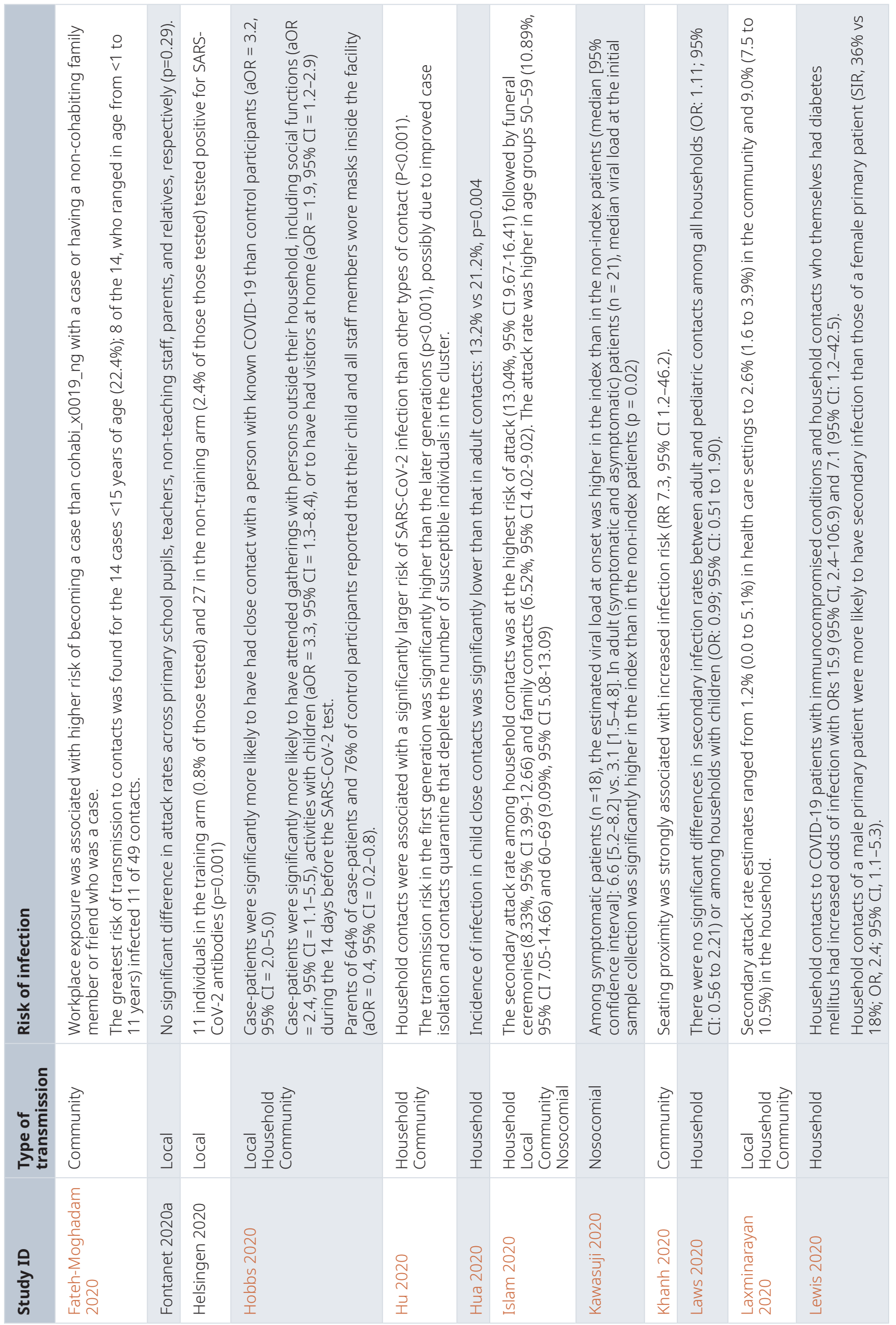




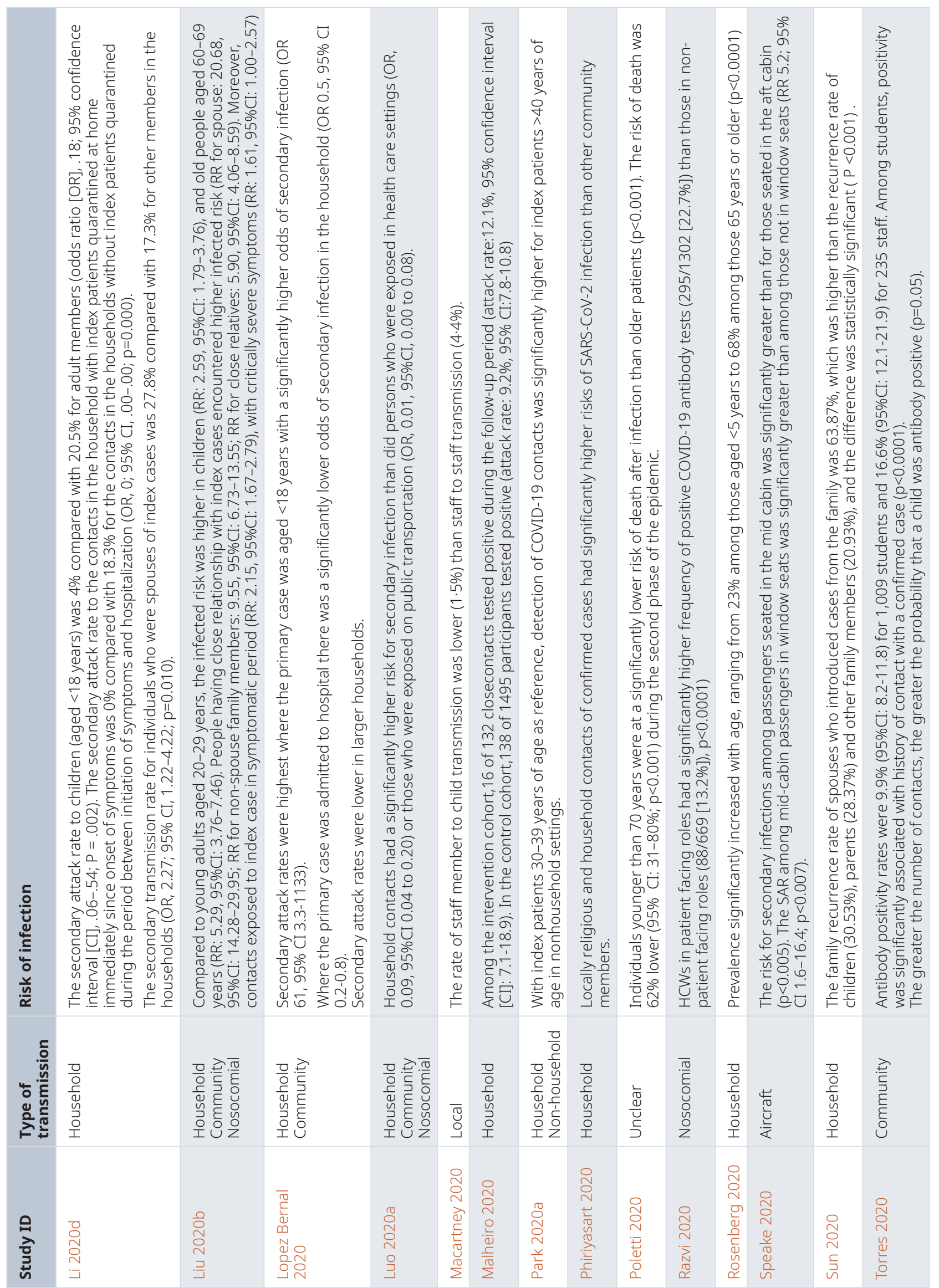




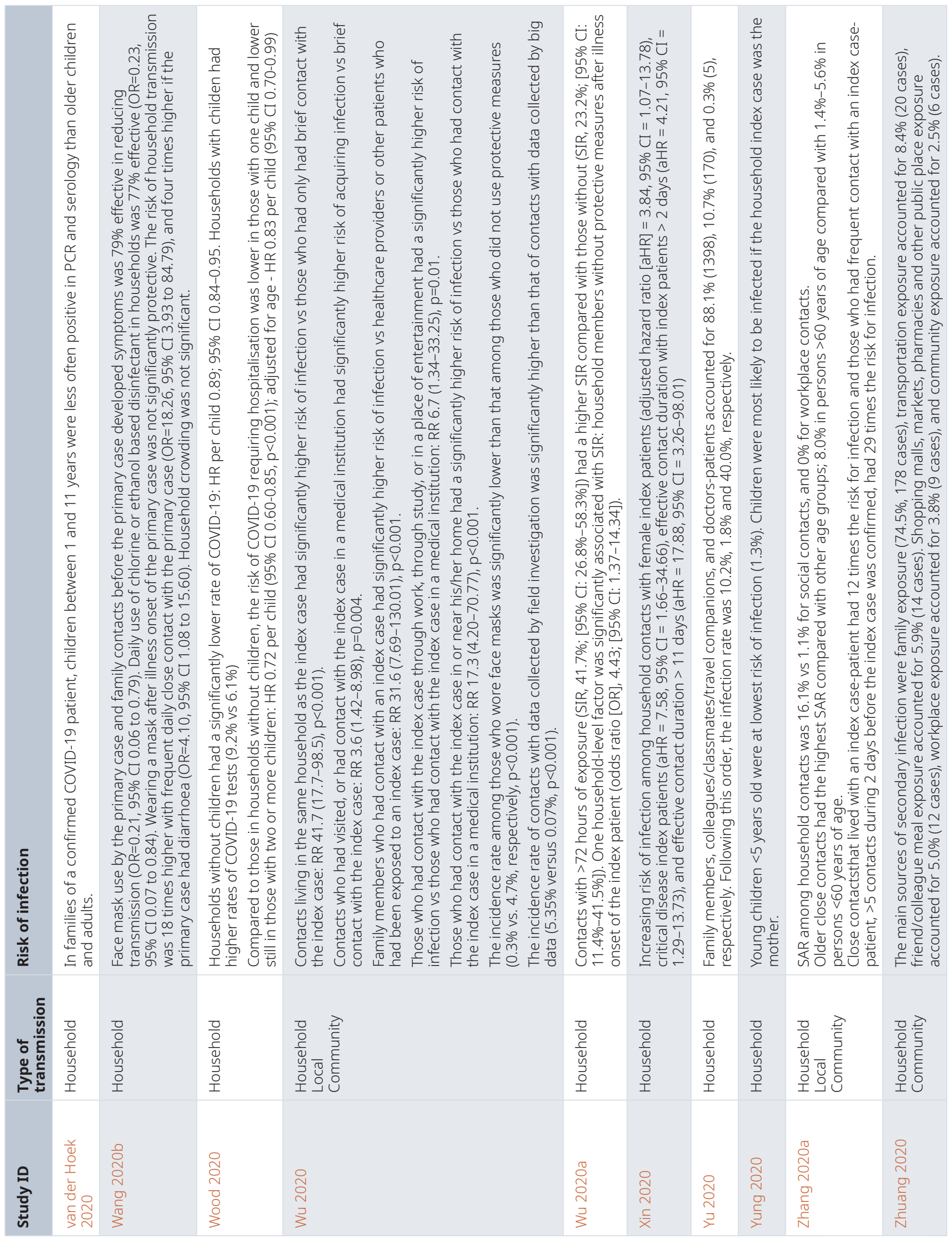


(Bi 2020a, Wu 2020, Zhang 2020a) showed that the more frequent contacts with an index case was significantly associated with an increased risk of infection.

\section{Viral culture}

Three studies (Ladhani 2020a, Speake 2020, Yang 2020) performed viral culture (Table 8). All studies utilised Vero E6 cells for viral culture. In Ladhani 2020a (a study of elderly nursing home residents), positive samples with a $\mathrm{Ct}$ of $<35$ were incubated on Vero E6 cells and confirmed by cytopathic effect (CPE) up to 14 days post-inoculation. Positive culture results were obtained for symptomatic, post-symptomatic, pre-symptomatic and asymptomatic cases (21 residents and 12 staff); higher $\mathrm{Ct}$ values was significantly associated with decreasing ability to recover the virus $(\mathrm{p}<0.001)$. Among residents the virus was isolated 12 days before symptom onset and up to 13 days after and in staff up to 6 days before and 7 days after symptom onset. In Speake 2020, specimens were inoculated in Vero-E6 cells and inspected for CPE daily for up to 10 days with identity confirmed using "in-house" PCRs. The primary cases had boarded the flight from a cruise ship and had SARSCoV-2 with the strain A2-Ruby Princess (A2-RP). Nine of 17 (53\%) of PCR-positive samples grew SARS-CoV-2 in culture. Eight secondary cases who were in the same flight cabin with the infected travellers from the cruise ship all had viruses of the A2-RP strain ( 3 by full and 1 by partial sequence) (Table 8). In the third study of index patients with recurrent infection swab specimens were also inoculated on Vero cells, and monitored for CPE daily for 10 days (Yang 2020). All four viral cultures were negative $(0 \%)$.

\section{Genome sequencing (GS) and phylogenetic analysis}

Ten studies (Böhmer 2020, Firestone 2020, Jiang 2020, Ladhani 2020a, Lucey 2020, Pung 2020, Sikkema 2020, Speake 2020, Taylor 2020, Wang 2020) performed GS and phylogenetic analysis (Table 9). The studies were primarily conducted in outbreak clusters and methods used for performing these investigations were essentially similar across the studies. The completeness of genomic similarity ranged from $81-100 \%$ across six studies (Firestone 2020, Jiang 2020, Lucey 2020, Sikkema 2020, Speake 2020, Wang 2020). Transmission from one case to a contact was demonstrated by nonsynonymous nucleotide polymorphism in SARS-CoV-2 from these two cases onwards, but not in any cases detected prior to this instance (Böhmer 2020). In one study of skilled nursing home facilities (Taylor 2020), samples from 75 residents and five healthcare staff shared genetically related strains. In another study of care homes (Ladhani 2020a), reported nine separate introductions of SARS-CoV-2 into care homes by healthcare staff. In one study which used multiple settings (Pung 2020), the viral genomic sequences for four cases in one cluster shared identical sequences over the full genome length and shared a common base difference relative to the earlier sequences (see Table 8).

\section{Discussion}

\section{Summary of main findings}

We identified 171 primary studies assessing the role of close contact in transmission of SARS-CoV-2. The evidence from these observational studies suggest that the risk of transmission is significantly increased through close contact with an infected case - the greater the frequency of contact, the greater the risk. Household contact setting is significantly more likely to result in transmission of SARS-CoV-2 compared to other types of contact settings. This risk of transmission appears to decrease with use of face masks and in cases where the index or primary cases are in the paediatric age group. The risk of close contact transmission is significantly increased in the elderly. Enclosed environments and social gatherings appear to increase the likelihood of close contact transmission. Close contact with persons having recurrent infection with SARS$\mathrm{CoV}-2$ is unlikely to result in transmission of the virus. There is wide heterogeneity in study designs and methods and the overall quality of evidence from published primary studies is sub-optimal. The results of systematic reviews also suggest that household contact setting increases the risk of transmission and being elderly is also associated with increased risks of transmission and mortality.

The positive results of viral cultures observed in two studies support the results of PCR and serologic tests showing that close contact setting was associated with transmission of SARS-CoV-2. The failure to successfully isolate the virus in the third study supports the view that individuals who are re-infected are unlikely to transmit the virus in close contact settings. The positive findings from all 10 studies that performed GS and phylogenetic analysis with identical strains supports the hypothesis that close contact setting is associated with SARS-CoV-2 transmission through respiratory droplets or direct contact. The failure of the majority of studies to report $\mathrm{Ct}$ values casts doubts on the strengths of any reported associations because of the likelihood of false positives, as is the lack of (and variation in) reporting of the timelines for sample collections. The variations observed in the definitions of close contacts also cast further doubts on the validity of overall results.

\section{Comparison with the existing literature}

The results of our review are consistent with several guidelines suggesting that close contact with index cases can result in transmission of SARS-CoV-2 ${ }^{8-10}$. Our findings are also consistent with those of a systematic review which concluded that face masks are effective for preventing transmission of respiratory viruses ${ }^{11}$. The results of our review also support those of a previous review which showed that the elderly are at increased risk of infection and mortality with coronavirus ${ }^{12}$. However, our review contains a greater number of studies compared to each of the included individual reviews and shows evidence demonstrating positive culture of virus as well as genomic evidence of close contact transmission. This differs from the findings from our reviews of fomite, orofecal and airborne transmission that failed to show evidence of either positive culture or genomic sequences demonstrating SARS-CoV-2 transmission ${ }^{13-15}$

\section{Strengths and limitations}

To our knowledge, this is the most comprehensive review to date investigating the role of close contact in the transmission of SARS-CoV-2. We extensively searched the literature for eligible studies, accounted for the quality of included studies and have reported outcomes (viral culture and GS) that 


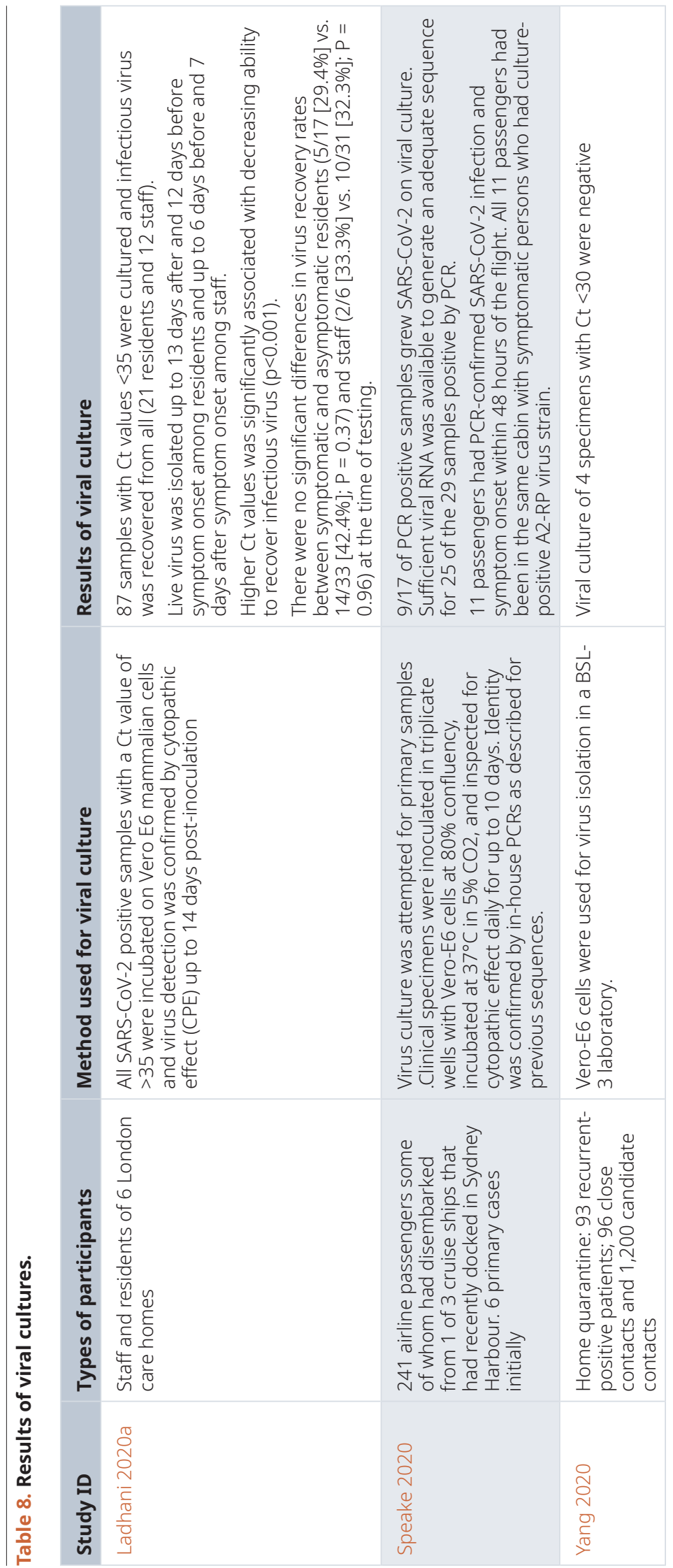




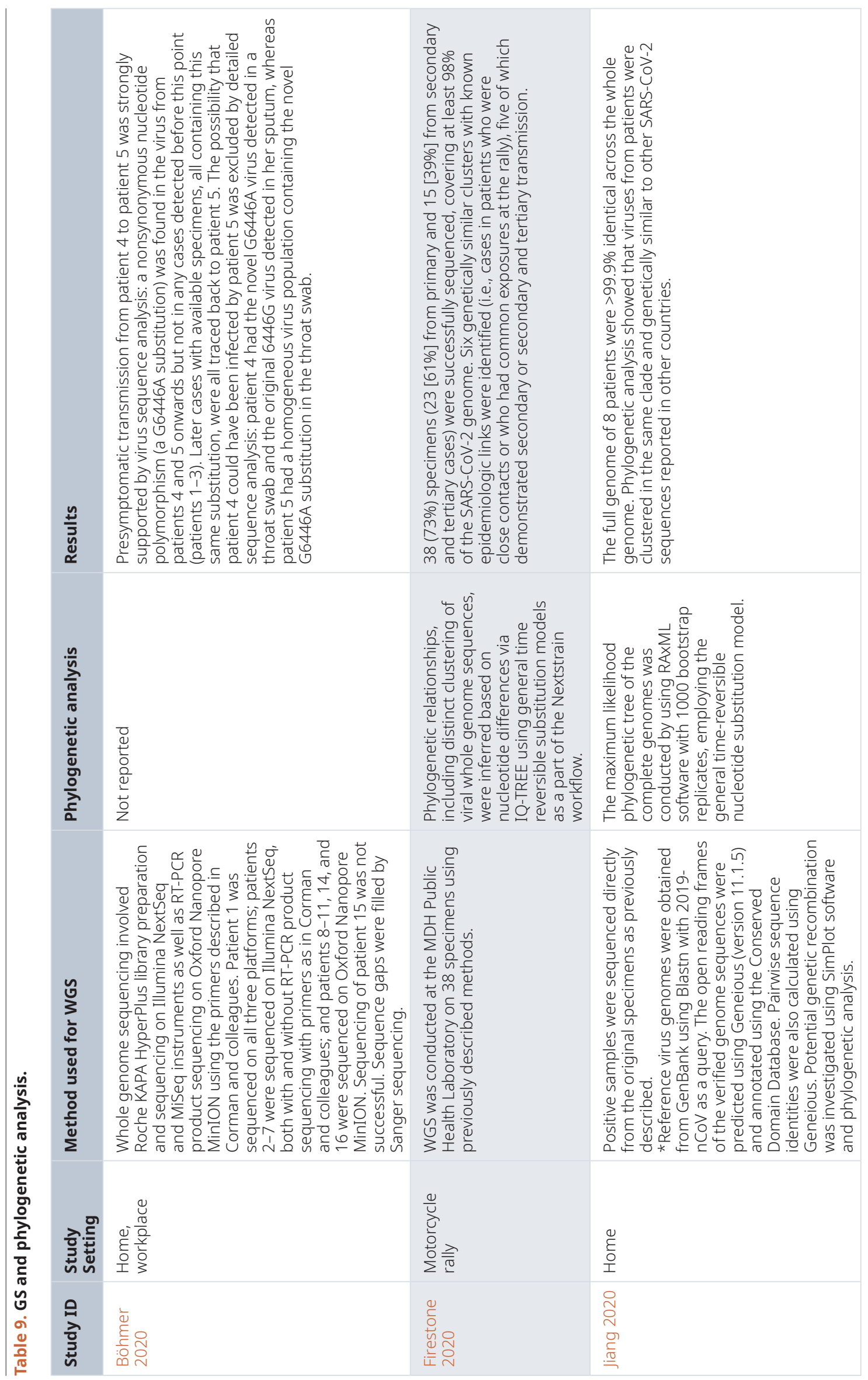




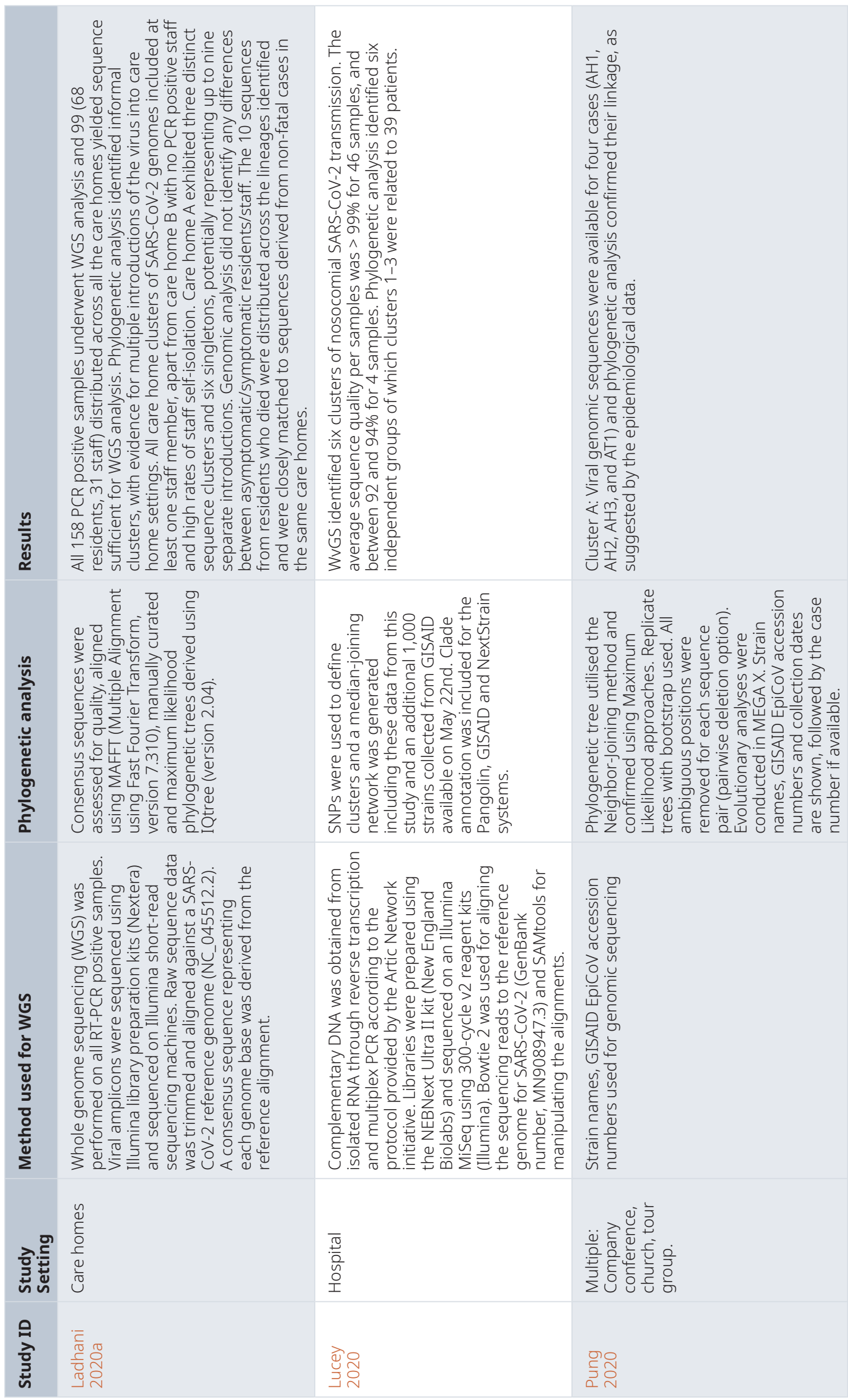




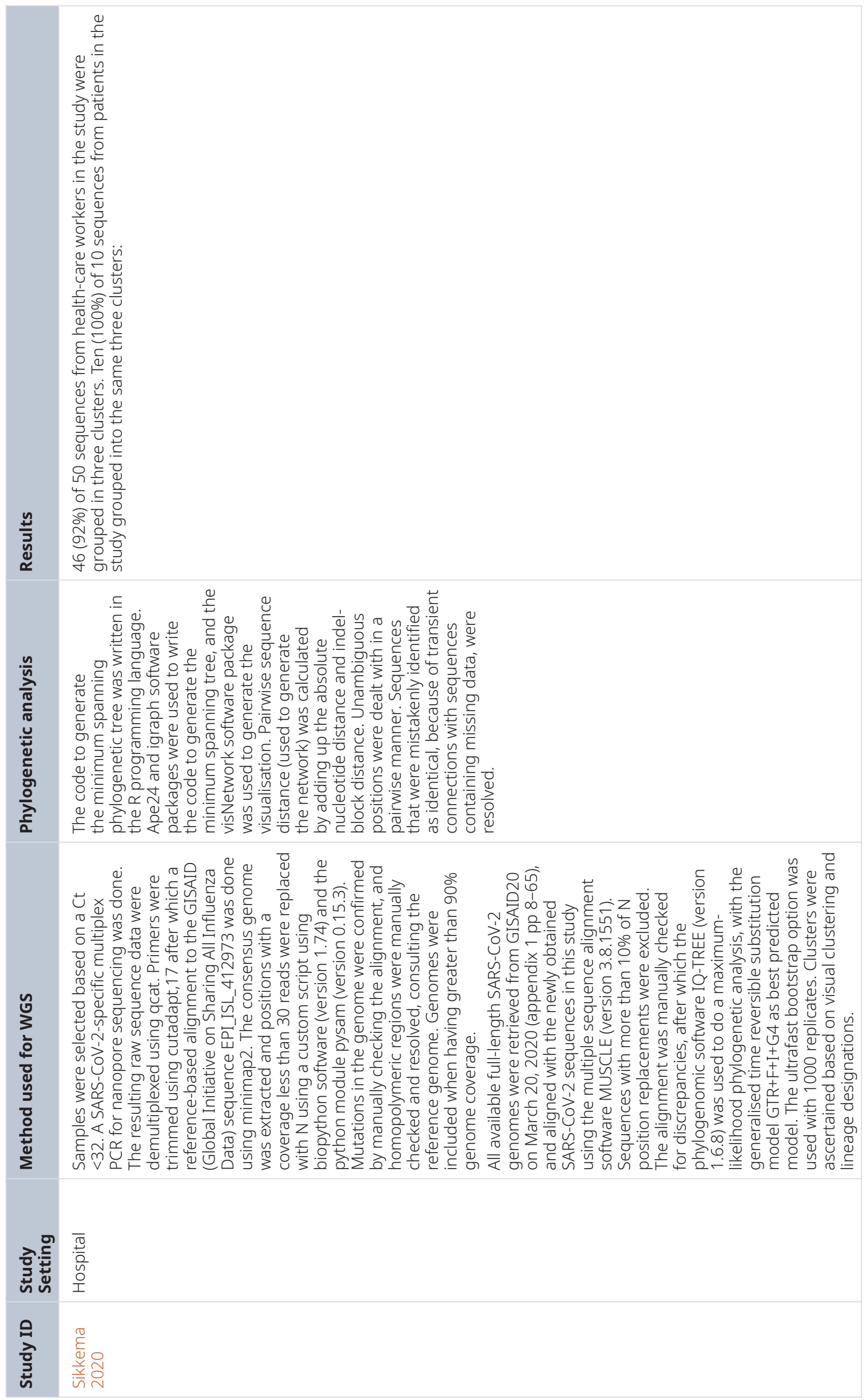




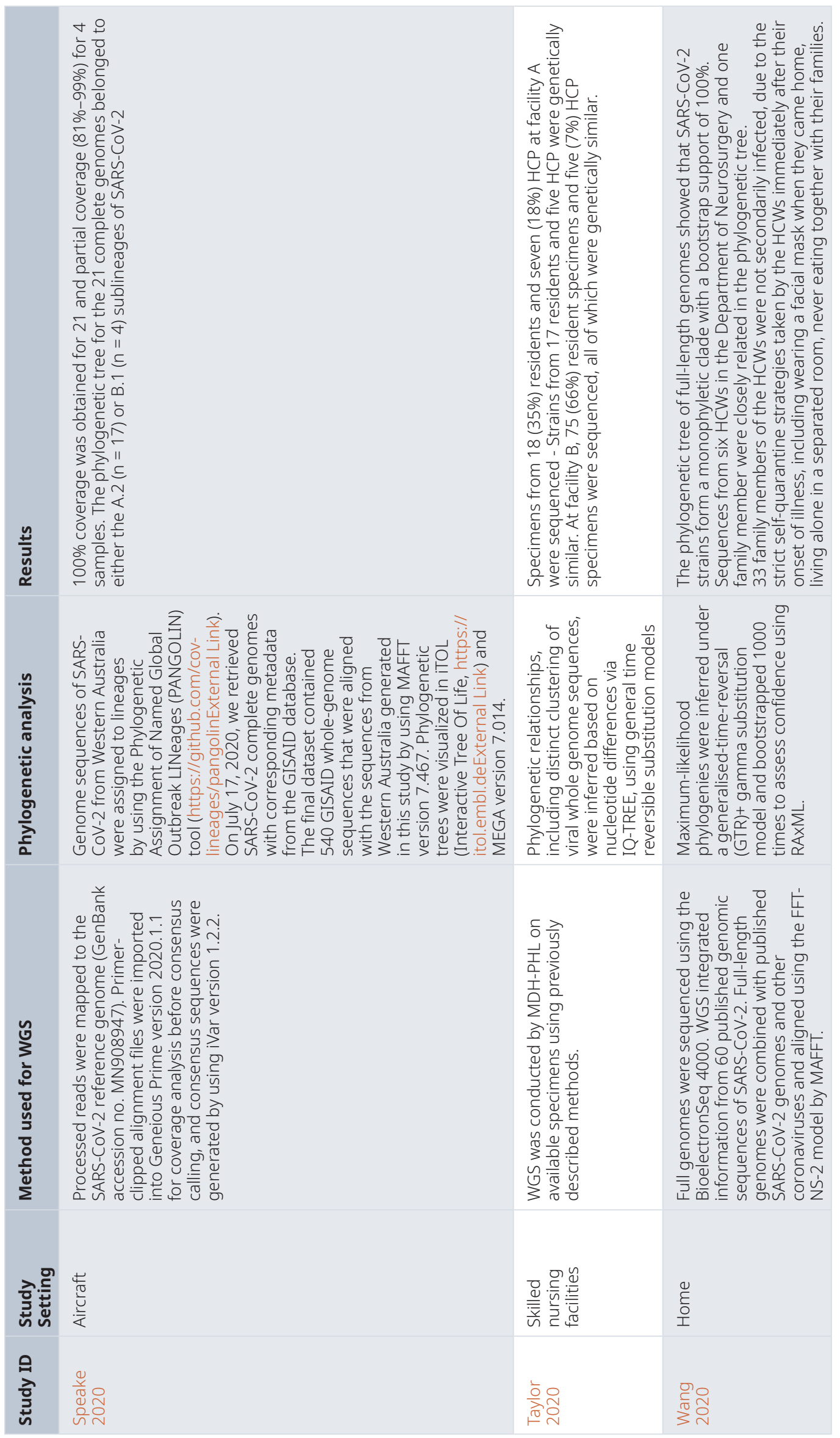


were previously unreported in previous reviews. However, we recognize some limitations. We may not have identified all relevant studies examining the role of close contact in transmission - this is especially true for unpublished studies. We included results from non-peer reviewed studies which may affect the reliability of the review results. However, such studies could potentially be of research benefit because of the ongoing pandemic; in addition, we performed forward citation search of relevant studies.

\section{Implications for research}

Future studies should endeavour to include $\mathrm{Ct}$ values (or preferably convert the $\mathrm{Ct}$ values to number of genome copies using standard curves) when reporting research results and should describe the timing and methods of sample collection. Details surrounding the proximity, timing, and activities within the context of close contact need to be described. In studies of elderly subjects, more detailed description of baseline demographics should be reported. Further studies showing virus isolation in close contact settings should be conducted to strengthen the current evidence base; this could include performing serial cultures. Similarly, more research examining genomic sequences and phylogenetic trees in suspected close contact transmissions should be conducted - this should also extend to research examining other modes of transmission. The variation in methods and thresholds of the serological tests add to the confusion about diagnostic accuracy of testing; indeed, some authors have questioned the value of serological tests for diagnosing SARS-CoV-2 ${ }^{16}$. To overcome the challenge of interpreting antibody responses, guidelines for better reporting of serological tests and results should be developed; this has previously been emphasized by other authors. Internationally recognized research dictionary of terms defining and describing close contact settings should be developed. Standardized guidelines for reporting research results should be a priority. Local, national, and international health organisations should promote good hygiene measures including advising against close contact with SARS-CoV-2 infected individuals; use of medical masks should be encouraged in circumstances where close contact with infected cases is likely. Activities in enclosed settings should be discouraged and social distancing in close contact settings should be encouraged.

\section{Conclusion}

The evidence from published observational studies and systematic reviews indicate that SARS-CoV-2 can be transmitted via close contact settings. Household contact and increased frequency of contact with infected cases significantly increase the risk of transmission. The quality of evidence from published studies is low-to moderate. Variations in study designs and methodology restrict the comparability of findings across studies. Standardized guidelines for the reporting of future research should be developed.

\section{Data availability}

Underlying data

All data underlying the results are available as part of the article and no additional source data are required.

\section{Extended data}

Figshare: Extended data: SARS-CoV-2 and the Role of Close Contact in Transmission: A Systematic Review, https://doi. org/10.6084/m9.figshare.14312630.v1 ${ }^{6}$.

This project contains the following extended data:

- Updated Protocol

- Search Strategy

- List of Excluded Studies

- References to Included Studies

Data are available under the terms of the Creative Commons Attribution 4.0 International license (CC-BY 4.0).

\section{Acknowledgements}

This work was commissioned and paid for by the World Health Organization (WHO). Copyright on the original work on which this article is based belongs to WHO. The authors have been given permission to publish this article. The author(s) alone is/are responsible for the views expressed in the publication. They do not necessarily represent views, decisions, or policies of the World Health Organization.
1. World Health Organization: WHO Coronavirus Disease (COVID-19) Dashboard. [Last Accessed 20/03/2021]. Reference Source

2. World Health Organization: Modes of transmission of virus causing COVID-19: implications for IPC precaution recommendations. [Accessed 16/01/2021]

Reference Source

3. World Health Organization: Coronavirus disease 2019 (COVID-19) Situation Report - 73. [Accessed 28 February 2021]. Reference Source
4. Tanne JH: Covid-19: CDC publishes then withdraws information on aerosol transmission. BMJ. 2020; 370: $\mathrm{m} 3739$.

PubMed Abstract | Publisher Full Text

5. Tang JW: SARS-CoV-2 and aerosols-Arguing over the evidence. J Virol Methods. 2021; 289: 114033.

PubMed Abstract | Publisher Full Text | Free Full Text

6. Onakpoya I, Heneghan C, Spencer E, et al.: Extended data: SARS-CoV-2 and the Role of Close Contact in Transmission: A Systematic Review. figshare. Figure. 2021

http://www.doi.org/10.6084/m9.figshare.14312630.v1 
7. Whiting PF, Rutjes AWS, Westwood ME, et al:: QUADAS-2: a revised tool for the quality assessment of diagnostic accuracy studies. Ann Intern Med. 2011; 155(8): 529-36.

PubMed Abstract | Publisher Full Text

8. World Health Organization: Q\&A: How is COVID-19 transmitted? [Accessed 06/04/2021].

Reference Source

9. Center for Disease Control and Prevention: COVID-19 Overview and Infection Prevention and Control Priorities in non-US Healthcare Settings. Updated Feb. 26, 2021. Reference Source

10. Public Health England: Guidance 12. COVID-19 infection prevention and control guidance: glossary of terms. Updated 21 January 2021. Reference Source

11. Liang M, Gao L, Cheng C, et al.: Efficacy of face mask in preventing respiratory virus transmission: A systematic review and meta-analysis. Travel Med Infect Dis. 2020; 36: 101751.

PubMed Abstract | Publisher Full Text | Free Full Text
12. Park JE, Jung $S$, Kim A, et al:: MERS transmission and risk factors: a systematic review. BMC Public Health. 2018; 18(1): 574. PubMed Abstract | Publisher Full Text | Free Full Text

13. Onakpoya IJ, Heneghan CJ, Spencer EA, et al.: SARS-CoV-2 and the role of fomite transmission: a systematic review [version 1; peer review: awaiting peer review]. F1000Res. 2021; 10: 233 . Publisher Full Text

14. Heneghan CJ, Spencer EA, Brassey J, et al.: SARS-CoV-2 and the role of orofecal transmission: a systematic review [version 1; peer review: awaiting peer review]. F1000Res. 2021; 10: 231 Publisher Full Text

15. Heneghan C, Spencer EA, Brassey J, et al:: SARS-CoV-2 and the role of airborne transmission: a systematic review [version 1; peer review: awaiting peer review]. F1000Res. 2021; 10: 232 Publisher Full Text

16. Bastos ML, Tavaziva G, Abidi SK, et al.: Diagnostic accuracy of serological tests for covid-19: systematic review and meta-analysis. BMJ. 2020; 370: tests for

m2516.
PubMed Abstract | Publisher Full Text | Free Full Text 


\section{Open Peer Review}

\section{Current Peer Review Status: $\mathrm{X}$ ?}

\section{Version 1}

Reviewer Report 05 April 2022

https://doi.org/10.5256/f1000research.55716.r123867

(C) 2022 Wamai R. This is an open access peer review report distributed under the terms of the Creative Commons Attribution License, which permits unrestricted use, distribution, and reproduction in any medium, provided the original work is properly cited.

\section{Richard Wamai \\ Integrated Initiative for Global Health, Northeastern University, Boston, MA, USA}

I have read this manuscript with keen interest and over several weeks during which COVID-19 has continued to evolve with new studies coming out and policy changes across countries I have been traveling in (Kenya and US). This manuscript deals with the question of availability of evidence for role of close contact in COVID-19 transmission. This question seems obvious now given 2+ years of generalized policy around the world for physical or social distancing. A lay observation would be to say that there is unequivocal evidence that close proximity has a role in the transmission of COVID (after all we have been told to distance). The authors of the present manuscript deal with the question of availability of scientific evidence for this generalized policy.

Clearly, a lot (if not all) of the NPIs (non-pharmaceutical interventions) have been implemented without evidence whether they work (Halperin DT, Hearst N, Hodgins S, et al. (2021) ${ }^{1}$ - I am a coauthor in this study. The current manuscript shows just how murky the state of evidence of these NPIs is. Consider the state of evidence of mask-wearing; just the Bangladeshi study is the only RCT we have, and the evidence that masks work from this trial is not that great (Abaluck J, Kwong LH, Styczynski A, et al., 2022²)

In my view, assessment of the merits of this manuscript should center on two things. The first is whether the authors have identified all the evidence available as published in scientific literature. The second is whether the authors make good-faith representation of the studies they have reviewed. I say "good-faith" because there are questions raised by the existing reviews and comments of this manuscript. Additionally, "good-faith" means to me that the authors have not omitted results from the studies. On these two accounts I write my recommendation. A systematic/scoping review is a unique type of study because authors should be presenting in good faith the information from the studies reviewed.

\section{Have the authors examined all of the available evidence (=published studies) on the question?}

The authors both say they have and have documented and included the method of their search 
for the literature reviewed (Appendix 2). The only problem I see with this is that studies reviewed were only those published as of December 20, 2020. That was the first full year of the pandemic. Publications from 2021 are not included. One would presume that it is more likely that more studies on the effect of distancing could have been conducted in 2021. Those studies are not included in this manuscript, and that is a shortcoming. One - and at the minimum necessary revision that could be made is to add the timeframe of the review in the Title of the manuscript. In my view, it is not necessary for the authors to begin including the studies from 2021 as that would require a full new search and a re-write of large potions of the manuscript.

\section{Have the authors presented truthfully and good-faith the evidence from the studies?}

This is the central question for this manuscript, and it appears more so the case given the extensive comments by the readers. In my view, all of the criticism is not warranted. Criticism is due where the authors have not presented results from the studies reviewed truthfully and in good-faith. Criticism is also due where gaps in evidence is not acknowledged or are pertaining. I do not think the readers present counter evidence, i.e., show that the authors have summarized results from a specific study or set of studies erroneously. In the same vein I am not convinced that readers demonstrate that the authors have misinterpreted results from the studies they have reviewed; such misinterpretation would be strong grounds for Not Approving this manuscript or calling for its revisions, of course. If the readers are presenting evidence to counter the evidence from the studies presented by the authors from other studies that is acceptable. But that should also be weighed against my point 1 above. For instance, could the initial search have missed such studies raised by the readers?

Also, I would not engage in some of the debates presented. For instance, I do not think the manuscript should be criticized because "these authors may lack key expertise in their team". I have read through the contributors' listed expertise. I do not think any special expertise above and beyond what the author team has is needed to conduct a systematic review or truthfully and in good-faith summarize the evidence from the reviewed studies. In addition, I do not think that simply because persons in the author team are members of a decision-making body like the WHO that disqualifies them from objectivity or removes their training in science and scholarship. On the contrary, I think there is great value when decision-makers also participate in science. The obvious problem, of course, is where there are conflicts of interests, or where such persons are compromised. However, we have to take the consideration that in a multi-authored manuscript not one of the authors influences the entire narrative of the manuscript or results presented. Persons whose employment or affiliation positions may - or may be perceived to - compromise any perspectives presented in a manuscript they have co-authored should of course declare the conflicts of interests. Declaration of conflicts of interests is a standard protocol required in journal standards.

Having said the above, I have a few other observations or questions of my own.

Q1: On table 5, many studies are of no assessed quality, i.e., miss the quality measures specified in this table. What if studies missing all (or even just 2) of these measures are excluded in the analysis? So, if only those applicable as judged in Fig 2 are included.

Q2: On page 46, "Risk of Infection". Is not 'risk of infection' similar to AR (attack rate) in the sense that AR reports risk of infection? In this section too (with Fig 3a) we would expect AR and SAR 
results to indicate risk of infection. Where, correspondingly, ARs and SARs are higher than risk of infection is high. Thus, risk of infection analysis do not present different results. Is this not how this should be understood?

Q3: "Implications for Research", page 58. The text, "Local, national, and international health organisations should promote good hygiene measures including advising against close contact with SARS-CoV-2 infected individuals; use of medical masks should be encouraged in circumstances where close contact with infected cases is likely. Activities in enclosed settings should be discouraged and social distancing in close contact settings should be encouraged." - To me, and objectively assessing the timing of the pandemic where we are today (April $1^{\text {st }}$ 2022), this text is mostly 'water under the bridge' and should thus be updated - if this manuscript is to be indexed. See discussions, e.g., in Halperin DT, Hearst N, Hodgins S, et al. (2021)1.

Additionally, I am not convinced the authors have presented compelling evidence here to warrant prompting such measures this text I highlight here include. The authors should strictly adhere to the evidence of their manuscript; it is wrong for the authors to echo the chorus of songs we have heard about these NPIs when there is no or limited evidence they work. Thirdly, as a further reason to exclude this concluding text, in this manuscript there is no discussion about hygiene. Evidence presented does not show close contact be avoided whatever the case. For instance, relevant studies reviewed show no transmission from (index) persons with previous repeated infection. Given this and the fact that by now many (if not most) people will have been infected (i.e., generalized spread) even multiple times (e.g., CDC. Nov. 16, 2021. Estimated COVID-19 Burden, https://www.cdc.gov/coronavirus/2019-ncov/cases-updates/burden.html), should we still be advocating for quarantine and masking? We argue for adherence to evidence (Halperin DT, Hearst N, Hodgins S, et al., $2021^{1}$.

Finally, there will be a few grammatical errors that should be checked.

I am grateful for the opportunity to read this manuscript and share my observations which I hope will help guide the authors in determining merit worthiness for indexing as well as contribute to continued discussions.

\section{References}

1. Halperin D, Hearst N, Hodgins S, Bailey R, et al.: Revisiting COVID-19 policies: 10 evidence-based recommendations for where to go from here. BMC Public Health. 2021; 21 (1). Publisher Full Text 2. Abaluck J, Kwong LH, Styczynski A, Haque A, et al.: Impact of community masking on COVID-19: A cluster-randomized trial in Bangladesh.Science. 2022; 375 (6577): eabi9069 PubMed Abstract I Publisher Full Text

\section{Are the rationale for, and objectives of, the Systematic Review clearly stated?} Yes

Are sufficient details of the methods and analysis provided to allow replication by others? Yes

Is the statistical analysis and its interpretation appropriate?

Not applicable 
Are the conclusions drawn adequately supported by the results presented in the review? Yes

Competing Interests: No competing interests were disclosed.

Reviewer Expertise: Global public health

I confirm that I have read this submission and believe that I have an appropriate level of expertise to confirm that it is of an acceptable scientific standard, however I have significant reservations, as outlined above.

Author Response 29 Jun 2022

IGHO ONAKPOYA, University of Oxford, Oxford, UK

Peer reviewer's comment: I have read this manuscript with keen interest and over several weeks during which COVID-19 has continued to evolve with new studies coming out and policy changes across countries I have been traveling in (Kenya and US). This manuscript deals with the question of availability of evidence for role of close contact in COVID-19 transmission. This question seems obvious now given $2+$ years of generalized policy around the world for physical or social distancing. A lay observation would be to say that there is unequivocal evidence that close proximity has a role in the transmission of COVID (after all we have been told to distance). The authors of the present manuscript deal with the question of availability of scientific evidence for this generalized policy.

Authors' response: Thank you.

Peer reviewer's comment: Clearly, a lot (if not all) of the NPIs (non-pharmaceutical interventions) have been implemented without evidence whether they work (Halperin DT, Hearst N, Hodgins S, et al. (2021)1 - I am a co-author in this study. The current manuscript shows just how murky the state of evidence of these NPIs is. Consider the state of evidence of mask-wearing; just the Bangladeshi study is the only RCT we have, and the evidence that masks work from this trial is not that great (Abaluck J, Kwong LH, Styczynski A, et al., 20222)

Authors' response: Thank you for this observation. As at the time we conducted the initial review, the evidence base was poor.

Peer reviewer's comment: In my view, assessment of the merits of this manuscript should center on two things. The first is whether the authors have identified all the evidence available as published in scientific literature. The second is whether the authors make goodfaith representation of the studies they have reviewed. I say "good-faith" because there are questions raised by the existing reviews and comments of this manuscript. Additionally, "good-faith" means to me that the authors have not omitted results from the studies. On these two accounts I write my recommendation. A systematic/scoping review is a unique type of study because authors should be presenting in good faith the information from the studies reviewed.

Authors' response: Thank you for focusing your peer review on the evidence as presented in the manuscript.

Peer reviewer's comment: 1 . Have the authors examined all of the available evidence 
(=published studies) on the question?

The authors both say they have and have documented and included the method of their search for the literature reviewed (Appendix 2). The only problem I see with this is that studies reviewed were only those published as of December 20,2020. That was the first full year of the pandemic. Publications from 2021 are not included. One would presume that it is more likely that more studies on the effect of distancing could have been conducted in 2021. Those studies are not included in this manuscript, and that is a shortcoming. One and at the minimum necessary - revision that could be made is to add the timeframe of the review in the Title of the manuscript. In my view, it is not necessary for the authors to begin including the studies from 2021 as that would require a full new search and a re-write of large potions of the manuscript.

Authors' response: We have updated the electronic searches up till 30/04/2022 because of the comments from reviewer \#1 and re-written several aspects of the manuscript to reflect this update.

Peer reviewer's comment: 2 . Have the authors presented truthfully and good-faith the evidence from the studies?

This is the central question for this manuscript, and it appears more so the case given the extensive comments by the readers. In my view, all of the criticism is not warranted.

Criticism is due where the authors have not presented results from the studies reviewed truthfully and in good-faith. Criticism is also due where gaps in evidence is not acknowledged or are pertaining. I do not think the readers present counter evidence, i.e., show that the authors have summarized results from a specific study or set of studies erroneously. In the same vein I am not convinced that readers demonstrate that the authors have misinterpreted results from the studies they have reviewed; such misinterpretation would be strong grounds for Not Approving this manuscript or calling for its revisions, of course. If the readers are presenting evidence to counter the evidence from the studies presented by the authors from other studies that is acceptable. But that should also be weighed against my point 1 above. For instance, could the initial search have missed such studies raised by the readers?

Authors' response: We thank the reviewer for making this point. We agree with you that the criticisms were not justified. We searched, identified, and analyzed the available evidence at that time point.

Peer reviewer's comment: Also, I would not engage in some of the debates presented. For instance, I do not think the manuscript should be criticized because "these authors may lack key expertise in their team". I have read through the contributors' listed expertise. I do not think any special expertise above and beyond what the author team has is needed to conduct a systematic review or truthfully and in good-faith summarize the evidence from the reviewed studies. In addition, I do not think that simply because persons in the author team are members of a decision-making body like the WHO that disqualifies them from objectivity or removes their training in science and scholarship. On the contrary, I think there is great value when decision-makers also participate in science. The obvious problem, of course, is where there are conflicts of interests, or where such persons are compromised. However, we have to take the consideration that in a multi-authored manuscript not one of the authors influences the entire narrative of the manuscript or results presented. Persons whose employment or affiliation positions may - or may be perceived to - compromise any 
perspectives presented in a manuscript they have co-authored should of course declare the conflicts of interests. Declaration of conflicts of interests is a standard protocol required in journal standards.

Authors' response: Again, we agree with the reviewer. We have enough expertise in our team and have co-authored hundreds of systematic reviews.

Peer reviewer's comment: Having said the above, I have a few other observations or questions of my own.

Authors' response: Thank you. We have responded to each observation.

Peer reviewer's comment: $\underline{\mathrm{Q} 1:}$ On table 5 , many studies are of no assessed quality, i.e., miss the quality measures specified in this table. What if studies missing all (or even just 2) of these measures are excluded in the analysis? So, if only those applicable as judged in Fig 2 are included.

Authors' response: If we removed studies missing all or even 2 domains, we think the overall quality would still be low to moderate. Only 9 studies adequately dealt with bias.

Peer reviewer's comment: Q2: On page 46, "Risk of Infection". Is not 'risk of infection' similar to AR (attack rate) in the sense that AR reports risk of infection? In this section too (with Fig 3a) we would expect AR and SAR results to indicate risk of infection. Where, correspondingly, ARs and SARs are higher than risk of infection is high. Thus, risk of infection analysis do not present different results. Is this not how this should be understood?

Authors' response: The AR is a crude measure of the rate of infection in a group. The risk of infection compares the rate of attacks between or across groups, based on other variables, e.g., seating proximity. (See Box 1).

Peer reviewer's comment: Q3: “Implications for Research", page 58. The text, "Local, national, and international health organisations should promote good hygiene measures including advising against close contact with SARS-CoV-2 infected individuals; use of medical masks should be encouraged in circumstances where close contact with infected cases is likely. Activities in enclosed settings should be discouraged and social distancing in close contact settings should be encouraged." - To me, and objectively assessing the timing of the pandemic where we are today (April 1st 2022), this text is mostly 'water under the bridge' and should thus be updated - if this manuscript is to be indexed. See discussions, e.g., in Halperin DT, Hearst N, Hodgins S, et al. (2021)1.

Authors' response: We understand the point the reviewer makes. These statements were based on our understanding at that time. We have revised this section with the updated evidence. We have included the suggested reference.

Peer reviewer's comment: Additionally, I am not convinced the authors have presented compelling evidence here to warrant prompting such measures this text I highlight here include. The authors should strictly adhere to the evidence of their manuscript; it is wrong for the authors to echo the chorus of songs we have heard about these NPIs when there is no or limited evidence they work. Thirdly, as a further reason to exclude this concluding text, in this manuscript there is no discussion about hygiene. Evidence presented does not show close contact be avoided whatever the case. For instance, relevant studies reviewed 
show no transmission from (index) persons with previous repeated infection. Given this and the fact that by now many (if not most) people will have been infected (i.e., generalized spread) even multiple times (e.g., CDC. Nov. 16, 2021. Estimated COVID-19 Burden, https://www.cdc.gov/coronavirus/2019-ncov/cases-updates/burden.html), should we still be advocating for quarantine and masking? We argue for adherence to evidence (Halperin DT, Hearst N, Hodgins S, et al., 20211.

Authors' response: Thank you for raising this issue and we agree that we should stay focused on the evidence found in our review. We have accordingly revised the text as above.

Peer reviewer's comment: Finally, there will be a few grammatical errors that should be checked.

Authors' response: Thanks. We have re-checked the manuscript for grammatical errors.

Peer reviewer's comment: I am grateful for the opportunity to read this manuscript and share my observations which I hope will help guide the authors in determining merit worthiness for indexing as well as contribute to continued discussions.

Authors' response: Thank you for your helpful feedback which we believe has helped to improve the quality of the manuscript.

Competing Interests: None

Reviewer Report 07 March 2022

https://doi.org/10.5256/f1000research.55716.r121151

(C) 2022 Escandón $\mathrm{K}$ et al. This is an open access peer review report distributed under the terms of the Creative Commons Attribution License, which permits unrestricted use, distribution, and reproduction in any medium, provided the original work is properly cited.

\section{Kevin Escandón}

Division of Infectious Diseases and International Medicine, University of Minnesota Medical School, Minneapolis, MN, USA

\section{Angela K. Ulrich}

${ }^{1}$ Center for Infectious Disease Research and Policy, University of Minnesota, Minneapolis, MN, USA

2 Division of Environmental Health Sciences, School of Public Health, University of Minnesota, Minneapolis, MN, USA

\section{General comments}

We commend the authors for attempting to conduct a systematic review on one of the most controversial topics related to the COVID-19 pandemic: SARS-CoV-2 transmission. This is an important effort that required much dedication and careful analysis. Unfortunately, we think this manuscript falls short of scientific quality and utility due to major methodologic and conceptual flaws. 
First, this systematic review in its current version fails to provide an accurate and updated picture of the existing evidence. We reviewed this manuscript in February 2022, two years into the pandemic, and while SARS-CoV-2 transmission remains a topic of great relevance, the picture regarding the modes of transmission is much clearer now than one year ago due to numerous epidemiologic and lab-based studies. Given this evidence, the WHO and the general scientific community agree that SARS-CoV-2 can be transmitted via droplet, short-range aerosol, long-range aerosol, and less frequently via fomites. This systematic review should be updated to reflect the most recent evidence.

Second, and most importantly, there are methodological flaws, conceptual concerns, and unsupported conclusions, as detailed below. Systematic reviews are designed to summarize evidence on specific questions or focused problems with pre-defined criteria to bring understanding and clarity through insightful analyses (even if no meta-analyses are conducted) of existing evidence. Close contact is not only poorly defined in most articles, but is actually addressed in less than a half of the articles included. Importantly, close contact is not a transmission mechanism itself, rather a feature of transmission mechanism(s). The authors erroneously conclude that studies that identified identical strains in close contacts using genome sequencing and phylogenetic analysis support transmission via respiratory droplets. The authors do not acknowledge that short-range aerosol transmission is also a possible (and a likely one) explanation.

Note that given the absence of line numbers, we are not providing numbered comments.

\section{Introduction}

The introduction should be updated as statistics are over one year old (March 2021).

The authors mention that the "spread of the virus appears to be slowing". This statement is not necessarily true considering the recent, recurring, and constantly evolving waves of infection attributed to increasingly transmissible variants of SARS-CoV-2. Furthermore, no citation is provided for this statement.

"Current evidence from epidemiologic and virologic studies suggest SARS-CoV-2 is primarily transmitted via respiratory droplets and direct and indirect contact". This sentence is not properly supported by current data; the authors rather cited two WHO 2021 resources. The authors must acknowledge airborne transmission - a route of transmission accepted by both WHO and CDC. Note that respiratory transmission of inhalable particles is the dominant mode of transmission, especially short-range. Indirect droplet / contact / fomite transmission is estimated to be minor.

Recommended references: Zhang \& Duchaine $2020^{1}$ and Leung $2021^{2}$.

The aim of this study is to assess the evidence from primary studies and existing systematic reviews investigating the role of close contact on SARS-CoV-2 transmission. This is aligned with the manuscript title ("SARS-CoV-2 and the role of close contact in transmission..."), the introduction, the methods, and figure and table titles. However, one major challenge is that the "close contact" framework is neither clearly defined in the literature nor is it often standardized in methods of primary studies. Authors address several aspects of transmission, from settings to distance, populations, testing/lab methods (PCR, serology, viral culture, GS and phylogenetic analysis), attack rates, risk of infection, etc. We agree that 
all of these are variables that influence or describe transmission. But close contact is only defined in $46.8 \%$ of included studies which causes concern over the use of consistently applied inclusion criteria. This systematic review seems to evaluate different aims and ends providing general descriptions of different subtopics related to transmission, not only to close contact. It is not appropriate for this study on the role of close contact to make such inference on the interaction between an infector and an infectee if not explicitly stated in the primary study.

\section{Terminology}

The authors seem to be using the CDC criteria but this is not clarified in the box definition and the citation link seems to be inadequate.

It should be "2 days prior to positive test" instead of "2 days prior to test specimen collection".

Some of the references are textbooks or web resources not easy to navigate or links are not active. Authors are encouraged to use the most relevant scientific articles on COVID-19 wherever possible.

Synonyms often used to close contact are close/short range and close proximity. The latter could be more beneficial since the word "contact" is often understood as physical and conspicuous encounters.

While it's understandable that definitions are needed to assess evidence, it is equally important to mention their limitations from the outset. For example, close contact is arbitrary for purposes of contact tracing-we know that the definition results in missing

\section{Methods} cases of exposure and infection at longer ranges.

The inclusion of articles identified via preprint servers is justifiable if the search criteria include very recent dates in an attempt to capture the most recent research. However, this systematic review only includes articles up to December 20th 2020 - if the authors choose to use this date or a recent one, articles on preprint servers should not be included.

The search strategy is not reproducible and more detail is required. For example, a detailed search strategy for the WHO COVID-19 database, LitCovid, medRxiv, and Google Scholar are not included in the Appendix 2. Nor is it clear exactly which keywords were used in the PubMed search, for example, were other terms used to capture the concept of duration and proximity of exposure?

It is not clear if authors defined "close contact" for inclusion in their systematic review. Additional detail is required to explain how the QUADAS- 2 tool was adapted for this study and if or how it was validated for this purpose.

https://figshare.com/articles/figure/Extended_data_SARS-CoV-

2_and_the_Role_of_Close_Contact_in_Transmission_A_Systematic_Review/14312630/1?file=27243050

The QUADAS-2 tool was designed for studies primarily designed as diagnostic accuracy studies and its use is likely to fall short to assess the quality of studies for this systematic review.

\section{Analyses of primary studies}


Although authors have included extended data containing the protocol and references to included and excluded studies, this remains outdated (March 2021). Several features are discussed and while some of them could suggest close contact transmission, there is quite a bit of heterogeneity in how these studies of transmission inform the role of close contact.

While we agree that close-contact transmission is a dominant feature of SARS-CoV-2 transmission through the inhalation of respiratory particles, this systematic review does not help advance the aim mentioned - understanding the role of close contact. The authors could revise this work so it separately addresses features of transmission using standardized or limited definitions for each one. Attribution of such analyses to close contact (i.e., its potential role) should result from the interpretation of such findings altogether rather than from "direct assessment of the impact of close contact in transmission" since most studies do not define or measure close contact systematically.

\section{Analyses of reviews}

The authors included 10 systematic reviews allegedly investigating close-contact SARS-CoV2 transmission. Some concerns make questionable the inclusion of such heterogeneous publications for an analysis that at best describes these almost individually without advancing the understanding of the role of close contact in SARS-CoV-2 transmission, which is the ultimate purpose of the authors' systematic review. It is expected that studies included in such publications are primarily observational, since randomized designs are complex and it remains unclear how research should ideally address gaps in our understanding of transmission. Findings of some of the included reviews (Li, Ludvigsson, Zhu) are not described with regard to close contact, but only to population groups; others are only mentioned with regard to asymptomatic infection or attack rates. We find this analysis in general unhelpful.

The authors analyze features different from close contact in the sections following "Primary studies". All these analyses are related to primary studies, so this should be reorganized for clarity. Again, there is a detailed description of studies that do not address close contact, e.g., "The result of one study (Rosenberg 2020) showed that the incidence of infection significantly increased with age $(p<0.0001)$, while those from another study (Poletti 2020) showed that being 70 years or older was associated with a significantly increased risk of SARS-CoV-2-related death $(p<0.001)$,"

\section{Discussion}

Authors should be careful in interpreting what their systematic review found, for instance that many studies report household transmission suggests that transmission occurs in indoor environments with higher exposure. Exposure results from concentration and time of contact with infectious respiratory particles. It, therefore, depends on a mix of frequency of contacts, range of contact, and infectiousness of index cases. But household transmission may perfectly encompass risk of infection beyond $3 \mathrm{~m}, 6 \mathrm{~m}$, or any other definition of close contact. We do expect that the risk of infection is greatest with the longest contact, though, but the definition of a close contact remains nebulous and the associated risk could vary depending on the environmental conditions that favor transmission. Because the purpose of this systematic review is to address transmission, the authors must discuss at least generally the interplay of these factors. SARS-CoV-2 transmission is a complex phenomenon that depends on the interaction between viral properties (infectious dose and infectivity correlates), the host and their features (breathing rate, respiratory tract morphology, target tissues, receptor distribution, host barriers, immune responses), and the environment 
(temperature, humidity, salinity, $\mathrm{pH}$, the medium or materials of the contaminated objects or surfaces, ventilation/airflow, ultraviolet radiation). Authors also should acknowledge that existing evidence suggests that transmission in close-contact settings is likely to be dominated by short-range respiratory inhalation of infectious virions.

The authors superficially mention the role of face masks in decreasing the risk of transmission from the pediatric population. This is a dangerous misinterpretation of evidence of two things that should be analyzed separately, i.e., 1) the efficacy of respiratory protection and 2) the risk of transmissibility from different populations. Certainly, this review was not designed to assess either of these aspects as a research question. As for respiratory protection, not all masks (or respirators) are expected to provide the same degree of protection. And if not correctly/consistently worn, they may not reduce risk. As for the usually overclaimed risk of children to transmit SARS-CoV-2, many potential confounders easily make this group appear highly contagious but it is unlikely this is due to intrinsic features of that population. Therefore, claims about it should be carefully framed to avoid stigmatization or unfair focalization and perceived efficacy of preventive strategies.

Discussion unrelated to close contact is seen, e.g., "being elderly is also associated with increased risks of transmission and mortality".

"The positive results of viral cultures observed in two studies support the results of PCR and serologic tests showing that close contact setting was associated with transmission of SARSCoV-2".

We are unsure how this manuscript supports this conclusion. Similarly, this conclusion is not supported by the data "The positive findings from all 10 studies that performed GS and phylogenetic analysis with identical strains supports the hypothesis that close contact setting is associated with SARS-CoV-2 transmission through respiratory droplets or direct contact."

We agree with the authors on the high heterogeneity of existing studies and that "The variations observed in the definitions of close contacts also cast further doubts on the validity of overall results."

"The results of our review are consistent with several guidelines suggesting that close contact with index cases can result in transmission of SARS-CoV-2"

The authors acknowledge that the evidence for close contact transmission is only low-to-moderate quality, thus, it is a stretch to say that close contact is consistently demonstrated as a risk factor. Guidelines have been evolving throughout the pandemic and while close contact may be associated with increased risk of transmission, this study could provide much stronger evidence if transmission properties were assessed in a clearly defined, systematic, and reproducible way. "Our findings are also consistent with those of a systematic review which concluded that face masks are effective for preventing transmission of respiratory viruses."

It is unclear how this systematic review regarding the role of close contract on transmission events contributes to the discussion regarding the role of face masks more description is needed to make this link explicit. Furthermore, the efficacy of face masks is a complex topic and confounded by a number of factors.

\section{Necessary updates}

Several of the preprints included in the systematic review and/or cited in the manuscript 
have been published; given that this manuscript is outdated and an update is needed, authors should take that opportunity to update preprints that have been published and make sure their findings remain unchanged and adjust accordingly. Some of the preprints now published are:

Helsingen $2020^{3}$

Paireau $2020^{4}$

Kuwelker $2020^{5}$

Lyngse $2020^{6}$

Jones $2020^{7}$

Chen $2020^{8}$

Fontanet $2020^{9}$

Armann $2020^{10}$

Charlotte $2020^{11}$

Angulo-Bazan $2020^{12}$

\section{References}

1. Zhang X, Duchaine C: SARS-CoV-2 and Health Care Worker Protection in Low-Risk Settings: a Review of Modes of Transmission and a Novel Airborne Model Involving Inhalable Particles. Clinical Microbiology Reviews. 2020; 34 (1). Publisher Full Text

2. Leung NHL: Transmissibility and transmission of respiratory viruses.Nat Rev Microbiol. 19 (8): 528-545 PubMed Abstract | Publisher Full Text

3. Helsingen L, Løberg M, Refsum E, Gjøstein D, et al.: Covid-19 transmission in fitness centers in Norway - a randomized trial. BMC Public Health. 2021; 21 (1). Publisher Full Text

4. Paireau J, Mailles A, Eisenhauer C, de Laval F, et al.: Early chains of transmission of COVID-19 in France, January to March 2020.Euro Surveill. 27 (6). PubMed Abstract | Publisher Full Text 5. Kuwelker K, Zhou F, Blomberg B, Lartey S, et al.: Attack rates amongst household members of outpatients with confirmed COVID-19 in Bergen, Norway: A case-ascertained study.Lancet Reg Health Eur. 2021; 3: 100014 PubMed Abstract | Publisher Full Text

6. Lyngse FP, Kirkeby C, Halasa T, Andreasen V, et al.: Nationwide study on SARS-CoV-2 transmission within households from lockdown to reopening, Denmark, 27 February 2020 to 1 August 2020.Euro Surveill. 27 (6). PubMed Abstract | Publisher Full Text

7. Jones $B$, Phillips $G$, Kemp S, Payne B, et al.: SARS-CoV-2 transmission during rugby league matches: do players become infected after participating with SARS-CoV-2 positive players?. $\mathrm{Br} J$ Sports Med. 2021; 55 (14): 807-813 PubMed Abstract | Publisher Full Text

8. Chen X, Chen Z, Azman A, Deng X, et al.: Serological evidence of human infection with SARS-CoV2: a systematic review and meta-analysis. The Lancet Global Health. 2021; 9 (5): e598-e609 Publisher Full Text

9. Fontanet A, Tondeur L, Grant R, Temmam S, et al.: SARS-CoV-2 infection in schools in a northern French city: a retrospective serological cohort study in an area of high transmission, France, January to April 2020.Euro Surveill. 26 (15). PubMed Abstract | Publisher Full Text 10. Armann JP, Kirsten C, Galow L, Kahre E, et al.: SARS-CoV-2 transmissions in students and teachers: seroprevalence follow-up study in a German secondary school in November and December 2020.BMJ Paediatr Open. 2021; 5 (1): e001036 PubMed Abstract | Publisher Full Text 11. Charlotte N: High Rate of SARS-CoV-2 Transmission Due to Choir Practice in France at the Beginning of the COVID-19 Pandemic.J Voice. 2020. PubMed Abstract | Publisher Full Text 12. Angulo-Bazán Y, Solis-Sánchez G, Cardenas F, Jorge A, et al.: Household transmission of SARS- 
CoV-2 (COVID-19) in Lima, Peru.Cad Saude Publica. 2021; 37 (3): e00238720 PubMed Abstract |

Publisher Full Text

Are the rationale for, and objectives of, the Systematic Review clearly stated?

Partly

Are sufficient details of the methods and analysis provided to allow replication by others?

No

Is the statistical analysis and its interpretation appropriate?

Partly

Are the conclusions drawn adequately supported by the results presented in the review? No

Competing Interests: No competing interests were disclosed.

Reviewer Expertise: Infectious diseases epidemiology, virology, public health.

We confirm that we have read this submission and believe that we have an appropriate level of expertise to state that we do not consider it to be of an acceptable scientific standard, for reasons outlined above.

Author Response 29 Jun 2022

IGHO ONAKPOYA, University of Oxford, Oxford, UK

Peer reviewers, comment: We commend the authors for attempting to conduct a systematic review on one of the most controversial topics related to the COVID-19 pandemic: SARS-CoV-2 transmission. This is an important effort that required much dedication and careful analysis. Unfortunately, we think this manuscript falls short of scientific quality and utility due to major methodologic and conceptual flaws.

Authors' response: We thank the reviewers for their positive comments and constructive criticisms. We have extensively revised the manuscript to address their concerns.

Peer reviewers' comment: First, this systematic review in its current version fails to provide an accurate and updated picture of the existing evidence. We reviewed this manuscript in February 2022, two years into the pandemic, and while SARS-CoV-2 transmission remains a topic of great relevance, the picture regarding the modes of transmission is much clearer now than one year ago due to numerous epidemiologic and lab-based studies. Given this evidence, the WHO and the general scientific community agree that SARS-CoV-2 can be transmitted via droplet, short-range aerosol, long-range aerosol, and less frequently via fomites. This systematic review should be updated to reflect the most recent evidence.

Authors' response: The review was submitted in March last year at the start of the pandemic; however, it took a long time before undergoing peer review. We have now updated the review to reflect the most recent evidence focused on the transmission 
associated with close contact. We updated our searches up till 30/04/2022.

Peer reviewers' comment: Second, and most importantly, there are methodological flaws, conceptual concerns, and unsupported conclusions, as detailed below. Systematic reviews are designed to summarize evidence on specific questions or focused problems with predefined criteria to bring understanding and clarity through insightful analyses (even if no meta-analyses are conducted) of existing evidence. Close contact is not only poorly defined in most articles, but is actually addressed in less than a half of the articles included. Importantly, close contact is not a transmission mechanism itself, rather a feature of transmission mechanism(s). The authors erroneously conclude that studies that identified identical strains in close contacts using genome sequencing and phylogenetic analysis support transmission via respiratory droplets. The authors do not acknowledge that shortrange aerosol transmission is also a possible (and a likely one) explanation.

Authors' response: We used the term "close contact settings" for our review, and we acknowledge variations in the definitions of close contact across the studies included in our review (see Table 3). We do not make any claims that close contact is a transmission mechanism but is associated with transmission from any of a number of mechanisms. We have added (with reference) that short-range aerosol transmission is a possible explanation for the identified identical strains in close contacts.

Peer reviewers' comment: Note that given the absence of line numbers, we are not providing numbered comments.

Authors' response: $\mathrm{OK}$

\section{Introduction}

\section{Peer reviewers' comment:}

The introduction should be updated as statistics are over one year old (March 2021).

The authors mention that the "spread of the virus appears to be slowing". This statement is not necessarily true considering the recent, recurring, and constantly evolving waves of infection attributed to increasingly transmissible variants of SARS-CoV-2. Furthermore, no citation is provided for this statement.

Authors' response: We have updated the searches to April 2022.

We have provided a citation to show that the infection rate is decreasing (

https://www.ons.gov.uk/peoplepopulationandcommunity/healthandsocialcare/conditionsanddiseases/articl ), and have also noted that the virus continues to evolve.

\section{Peer reviewers' comment:}

"Current evidence from epidemiologic and virologic studies suggest SARS-CoV-2 is primarily transmitted via respiratory droplets and direct and indirect contact". This sentence is not properly supported by current data; the authors rather cited two WHO 2021 resources. The authors must acknowledge airborne transmission - a route of transmission accepted by both WHO and CDC. Note that respiratory transmission of inhalable particles is the dominant mode of transmission, especially short-range. Indirect droplet / contact / fomite transmission is estimated to be minor.

Recommended references: Zhang \& Duchaine 20201 and Leung 20212.

Authors' response: We wish to thank the reviewer for this comment. We have updated the 
information and referenced the CDC and the WHO. The CDC statement suggests that exposure with infection occurs in 3 principal ways including inhalation of fine respiratory droplets, deposition of respiratory droplets and particles on exposed mucous membranes, splashes and sprays 'and touching mucous membranes with hands soiled by virus contained in respiratory fluids" (Scientific Brief: SARS-CoV-2 Transmission | CDC). They openly acknowledge the relative contributions of the modes of transmission outlined are unquantified and difficult to establish. We have revised the statement to state that the virus is primarily transmitted through exposure to infectious respiratory fluids such as fine aerosols, respiratory droplets, and added a further reference (

https://www.cdc.gov/coronavirus/2019-ncov/science/science-briefs/sars-cov-2transmission.html). The WHO states "available evidence continues to suggest that SARSCoV-2 can spread from an infected person's mouth or nose in small liquid particles when the person coughs, sneezes, sings, breathes or talks, by inhalation or inoculation through the mouth, nose or eyes. These liquid particles are different sizes, ranging from larger 'respiratory droplets' to smaller 'aerosols." Current evidence suggests that the virus spreads mainly between people who are in close contact with each other, typically within 1 metre, They also indicate that "the virus can also spread to others through aerosols at longer (beyond the typical 1 metre distance) distances. The risk of long-distance aerosol transmission is higher in poorly ventilated and/or crowded indoor settings" and further discuss transmission through fomites but acknowledge data is limited. Similar to the CDC they indicate the many challenges in working out the presence and transmission of infectious viruses. Rather than state the respiratory transmission of inhalable particles is the dominant mode of transmission we would prefer a more cautious scientifically based response and acknowledge the gap in knowledge in this area. Infection prevention and control during health care when coronavirus disease (COVID-19) is suspected or confirmed (who.int)

\section{Peer reviewers' comment:}

The aim of this study is to assess the evidence from primary studies and existing systematic reviews investigating the role of close contact on SARS-CoV-2 transmission. This is aligned with the manuscript title ("SARS-CoV-2 and the role of close contact in transmission..."), the introduction, the methods, and figure and table titles. However, one major challenge is that the "close contact" framework is neither clearly defined in the literature nor is it often standardized in methods of primary studies. Authors address several aspects of transmission, from settings to distance, populations, testing/lab methods (PCR, serology, viral culture, GS and phylogenetic analysis), attack rates, risk of infection, etc. We agree that all of these are variables that influence or describe transmission. But close contact is only defined in $46.8 \%$ of included studies which causes concern over the use of consistently applied inclusion criteria. This systematic review seems to evaluate different aims and ends providing general descriptions of different subtopics related to transmission, not only to close contact. It is not appropriate for this study on the role of close contact to make such inference on the interaction between an infector and an infectee if not explicitly stated in the primary study.

Authors' response: We thank the reviewer for this comment to which we have thought a great deal. We can respond by stating that although only $46.8 \%$ (now $39.1 \%$ ) defined close contact, the authors in the other included studies reported within their studies that they 
were investigating close contact. We acknowledge that not all studies provided precise definitions and that is a limitation. We set out to summarize the evidence from published studies that assess the association with close contact transmission in COVID-19.

As stated earlier, we have used the term "close contact settings" for our review.

\section{Terminology}

Peer reviewers' comment:

The authors seem to be using the CDC criteria but this is not clarified in the box definition and the citation link seems to be inadequate.

Authors' response: Thanks. We have revised the definition.

Peer reviewers' comment: It should be "2 days prior to positive test" instead of "2 days prior to test specimen collection"

Authors' response: Thanks. We have revised the text accordingly.

Peer reviewers' comment: Some of the references are textbooks or web resources not easy to navigate or links are not active. Authors are encouraged to use the most relevant scientific articles on COVID-19 wherever possible

Authors' response: We have used the updated citations for the relevant articles.

Peer reviewers' comment: Synonyms often used to close contact are close/short range and close proximity. The latter could be more beneficial since the word "contact" is often understood as physical and conspicuous encounters.

Authors' response: We have broadly used "close contact setting" to allow us to capture the range of studies assessing transmission characteristics of SARS-CoV-2. We appreciate the reviewers' suggestions of close proximity/range. However, these may be more useful for more focused review questions as they also have their limitations, e.g., direct contact would not be covered by these; however, direct contact has been described as a subset of close contact in some studies.

\section{Peer reviewers' comment:}

While it's understandable that definitions are needed to assess evidence, it is equally important to mention their limitations from the outset. For example, close contact is arbitrary for purposes of contact tracing-we know that the definition results in missing cases of exposure and infection at longer ranges.

Authors' response: Thank you for this suggestion. We have noted this in the limitations section.

\section{Peer reviewers' comment:}

The inclusion of articles identified via preprint servers is justifiable if the search criteria include very recent dates in an attempt to capture the most recent research. However, this systematic review only includes articles up to December 20th 2020 - if the authors choose to use this date or a recent one, articles on preprint servers should not be included.

Authors' response: We have updated the searches and used the most up-to-date citations for the included studies. 
Peer reviewers' comment: The search strategy is not reproducible and more detail is required. For example, a detailed search strategy for the WHO COVID-19 database, LitCovid, medRxiv, and Google Scholar are not included in the Appendix 2. Nor is it clear exactly which keywords were used in the PubMed search, for example, were other terms used to capture the concept of duration and proximity of exposure?

Authors' response: Thank you. We have included the full search strategy.

\section{Peer reviewers' comment:}

It is not clear if authors defined "close contact" for inclusion in their systematic review.

Authors' response: We used the CDC and WHO definitions (see Box 1).

Peer reviewers' comment: Additional detail is required to explain how the QUADAS-2 tool was adapted for this study and if or how it was validated for this purpose.

https://figshare.com/articles/figure/Extended_data_SARS-CoV-

2_and_the_Role_of_Close_Contact_in_Transmission_A_Systematic_Review/14312630/1?file $=27243050$

Authors' response: We did not use all the domains in QUADAS-2 and have clarified this in the manuscript methods section. We did not validate the checklist, and have noted this in our limitations.

\section{Peer reviewers' comment:}

The QUADAS-2 tool was designed for studies primarily designed as diagnostic accuracy studies and its use is likely to fall short to assess the quality of studies for this systematic review.

Authors' response: QUADAS-2 is used to assess reporting quality in diagnostic reviews. See https://www.bristol.ac.uk/media-

library/sites/quadas/migrated/documents/quadas2reportv4.pdf. As noted in our methods, we adapted the checklist for the review.

\section{Analyses of primary studies}

Peer reviewers' comment:

Although authors have included extended data containing the protocol and references to included and excluded studies, this remains outdated (March 2021). Several features are discussed and while some of them could suggest close contact transmission, there is quite a bit of heterogeneity in how these studies of transmission inform the role of close contact.

Authors' response: We have updated the searches and included the most up-to-date citations.

\section{Peer reviewers' comment:}

While we agree that close-contact transmission is a dominant feature of SARS-CoV-2 transmission through the inhalation of respiratory particles, this systematic review does not help advance the aim mentioned - understanding the role of close contact. The authors could revise this work so it separately addresses features of transmission using standardized or limited definitions for each one. Attribution of such analyses to close contact (i.e., its potential role) should result from the interpretation of such 
findings altogether rather than from "direct assessment of the impact of close contact in transmission" since most studies do not define or measure close contact systematically.

Authors' response: We included a table showing how the included studies defined close contact. Our objective was to identify, appraise and summarise the evidence from studies investigating transmission in close contact settings. It is equally possible that with close contact there may be transmission via large respiratory droplets and direct physical contact as well as the possibility of short-range fine aerosol and in all fairness, it should be stated as such and not that inhalation is the dominant route as this reviewer contends. It is best to be consistent with all possibilities as suggested by the CDC and WHO and many other publications.

\section{Analyses of reviews \\ Peer reviewers' comment:}

The authors included 10 systematic reviews allegedly investigating close-contact SARS-CoV-2 transmission. Some concerns make questionable the inclusion of such heterogeneous publications for an analysis that at best describes these almost individually without advancing the understanding of the role of close contact in SARSCoV-2 transmission, which is the ultimate purpose of the authors' systematic review. It is expected that studies included in such publications are primarily observational, since randomized designs are complex and it remains unclear how research should ideally address gaps in our understanding of transmission. Findings of some of the included reviews (Li, Ludvigsson, Zhu) are not described with regard to close contact, but only to population groups; others are only mentioned with regard to asymptomatic infection or attack rates. We find this analysis in general unhelpful.

Authors' response: Thanks for this comment. The included systematic reviews are considered appropriate since they do fit the definition of close contact based on our original protocol - this relates to structural and population settings. We can discuss the limitations of these reviews and the included studies. Li, Ludvigsson and Zhu fit the definitions. See https://www.ecdc.europa.eu/en/covid-19/surveillance/surveillance-definitions. Some of the findings from the reviews are helpful. Indeed at least 2 reviews included only studies with high reporting quality.

\section{Peer reviewers' comment:}

The authors analyze features different from close contact in the sections following "Primary studies". All these analyses are related to primary studies, so this should be reorganized for clarity. Again, there is a detailed description of studies that do not address close contact, e.g., "The result of one study (Rosenberg 2020) showed that the incidence of infection significantly increased with age $(p<0.0001)$, while those from another study (Poletti 2020) showed that being 70 years or older was associated with a significantly increased risk of SARS-CoV-2-related death $(p<0.001), "$

Authors' response: Thank you for pointing this out and we have revised the section on primary studies. We have enumerated the definitions of close contacts in the included studies in Table 3 and reported that several studies did not report a definition). Several studies showed that being elderly was significantly associated with increased risk of infection. Rosenberg 2020 included household contacts. We have revised the statement. 


\section{Discussion \\ Peer reviewers' comment:}

Authors should be careful in interpreting what their systematic review found, for instance that many studies report household transmission suggests that transmission occurs in indoor environments with higher exposure. Exposure results from concentration and time of contact with infectious respiratory particles. It, therefore, depends on a mix of frequency of contacts, range of contact, and infectiousness of index cases. But household transmission may perfectly encompass risk of infection beyond $3 \mathrm{~m}, 6 \mathrm{~m}$, or any other definition of close contact. We do expect that the risk of infection is greatest with the longest contact, though, but the definition of a close contact remains nebulous and the associated risk could vary depending on the environmental conditions that favor transmission. Because the purpose of this systematic review is to address transmission, the authors must discuss at least generally the interplay of these factors. SARS-CoV-2 transmission is a complex phenomenon that depends on the interaction between viral properties (infectious dose and infectivity correlates), the host and their features (breathing rate, respiratory tract morphology, target tissues, receptor distribution, host barriers, immune responses), and the environment (temperature, humidity, salinity, $\mathrm{pH}$, the medium or materials of the contaminated objects or surfaces, ventilation/airflow, ultraviolet radiation). Authors also should acknowledge that existing evidence suggests that transmission in close-contact settings is likely to be dominated by short-range respiratory inhalation of infectious virions.

Authors' response: Thank you. We have enumerated the complex range of factors at play and the epidemiologic associations in relation to close contact transmission (with references) and have acknowledged the various potential modes of transmission. We agree that transmission is a complex phenomenon and indeed is poorly understood. Musing about issues surrounding the biology of virus particles is beyond the scope of our review which focuses on epidemiologic associations.

Peer reviewers' comment: The authors superficially mention the role of face masks in decreasing the risk of transmission from the pediatric population. This is a dangerous misinterpretation of evidence of two things that should be analyzed separately, i.e., 1) the efficacy of respiratory protection and 2) the risk of transmissibility from different populations. Certainly, this review was not designed to assess either of these aspects as a research question. As for respiratory protection, not all masks (or respirators) are expected to provide the same degree of protection. And if not correctly/consistently worn, they may not reduce risk. As for the usually overclaimed risk of children to transmit SARS-CoV-2, many potential confounders easily make this group appear highly contagious but it is unlikely this is due to intrinsic features of that population. Therefore, claims about it should be carefully framed to avoid stigmatization or unfair focalization and perceived efficacy of preventive strategies.

Authors' response: We do not believe we are being superficial but rather trying to stay true to the findings. Our statements regarding the effectiveness of face masks are based on the findings from the included studies and we need to stay focused on the findings and avoid making speculative statements. We have added a caveat that there is uncertainty about the extent to which the different types of masks influence the risk of transmission. 
Peer reviewers' comment: Discussion unrelated to close contact is seen, e.g., "being elderly is also associated with increased risks of transmission and mortality".

Authors' response: Thank you for pointing this out. We have removed the statement.

\section{Peer reviewers' comment:}

The positive results of viral cultures observed in two studies support the results of PCR and serologic tests showing that close contact setting was associated with transmission of SARS-CoV-2".

We are unsure how this manuscript supports this conclusion. Similarly, this conclusion is not supported by the data "The positive findings from all 10 studies that performed GS and phylogenetic analysis with identical strains supports the hypothesis that close contact setting is associated with SARS-CoV2 transmission through respiratory droplets or direct contact."

Authors' response: We have revised the statement to indicate that transmission can occur in close contact settings.

Peer reviewers' comment: We agree with the authors on the high heterogeneity of existing studies and that "The variations observed in the definitions of close contacts also cast further doubts on the validity of overall results."

Authors' response: Thank you.

\section{Peer reviewers' comment:}

"The results of our review are consistent with several guidelines suggesting that close contact with index cases can result in transmission of SARS-CoV-2"

The authors acknowledge that the evidence for close contact transmission is only low-to-moderate quality, thus, it is a stretch to say that close contact is consistently demonstrated as a risk factor. Guidelines have been evolving throughout the pandemic and while close contact may be associated with increased risk of transmission, this study could provide much stronger evidence if transmission properties were assessed in a clearly defined, systematic, and reproducible way.

Authors' response: Thank you for this comment. We have revised the sentence to discuss the "association" with transmission as opposed to "can result in transmission". We have added a caveat to note that guidelines keep evolving based on emerging evidence.

\section{Peer reviewers' comment:}

"Our findings are also consistent with those of a systematic review which concluded that face masks are effective for preventing transmission of respiratory viruses."

It is unclear how this systematic review regarding the role of close contract on transmission events contributes to the discussion regarding the role of face masks - more description is needed to make this link explicit. Furthermore, the efficacy of face masks is a complex topic and confounded by a number of factors.

Authors' response: We have revised the statements. We have deleted the texts relating to mortality in the elderly.

\section{Peer reviewers' comment: Necessary updates}

Several of the preprints included in the systematic review and/or cited in the 
manuscript have been published; given that this manuscript is outdated and an update is needed, authors should take that opportunity to update preprints that have been published and make sure their findings remain unchanged and adjust accordingly. Some of the preprints now published are:

Helsingen 20203

Paireau 20204

Kuwelker 20205

Lyngse 20206

Jones 20207

Chen 20208

Fontanet 20209

Armann 202010

Charlotte 202011

Angulo-Bazan 202012

Authors' response: Thank you. We have now updated the citations for the references.

Competing Interests: None

\section{Comments on this article}

\section{Version 1}

Reader Comment 23 Jun 2021

Trish Greenhalgh, University of Oxford, Oxford, UK

\section{RESPONSE TO DR ONAKPOYA ET AL. TO THEIR RESPONSE (DATED 14TH JUNE 2021) TO OUR ORIGINAL COMMENT ON THEIR PAPER}

We thank Dr. Onakpoya et al. for the response to our comment. However, most of the points in our comment have not been addressed at all. They provided a longer response only to the virology comment, but all our other comments are just as important. Here we provide brief responses to each of the points in the response from Onakpoya et al., and call attention to the questions that are still missing a response.

\section{Authors' response to our comment \#1.}

The authors said: "Thank you. We have found conflicting definitions of particle size and the issue of short vs long range transmission is complex and requires a common set of agreed upon definitions and more study."

This response did not address the concern we raised in our original Comment 1, which pointed out that the possibility of short-range aerosol transmission being a major (and potentially dominant) contributor to "close contact" transmission was never mentioned in their review. We consider this a 
serious omission for the reasons set out in our original comment.

Regarding particle size: It is clear to aerosol scientists that the 5 micron separation between droplets and aerosols discussed in WHO's latest scientific brief (World Health Organization 2020) is very erroneous. The correct separation is approximately 100 microns, as originally reported by Wells (Wells 1934), confirmed by others (Xie et al. 2007) and recently reaffirmed by the US National Academies of Science, Engineering, and Medicine (Samet et al. 2021; Prather et al. 2020). The 5 micron error appears to have arisen at the CDC in the 1960s, by confusing the particle size that reaches the deep lung to infect in pulmonary tuberculosis with the particle size that falls to the ground in 1-2 meters from the infected person (Randall et al. 2021).

\section{Authors' response to our comment \#2.}

The authors said: "As we make clear in the text, we draw no definitive conclusions on this issue. We do not "believe" anything without solid evidence."

This response did not address the concern in our Comment 2, which was that equating ease of infection in close proximity with droplet transmission, as the authors appear to do in their paper, is a major conceptual error. For example, the following text appears in the paper:

"Current evidence from epidemiologic and virologic studies suggest SARS-COV-2 is primarily transmitted via respiratory droplets and direct and indirect contact."

How was that conclusion reached? Two WHO documents are cited, where the main justification for large droplet transmission is that transmission under close proximity is important. If the authors agree that no conclusion about the importance of droplet transmission can be reached from studies of transmission in close proximity, this should be clearly stated in the revised version.

In addition, the authors did not reply about whether a separate review on large droplet transmission is forthcoming. This is a topic of the utmost importance, since WHO maintains that large droplets are the main mode of SARS-CoV-2 transmission. It is very unclear what evidence supports that determination.

\section{Authors' response to our comment \#4.}

The authors said: "Once an agreed upon set of revised definitions is achieved, we and others would use them in any reviews of the evidence."

We respectfully suggest that the problem here is not one of agreed-upon definitions but of mechanisms of transmission. We question the authors' stance that the way viral transmission is conceptualised must remain flawed until everyone agrees on a definition of what counts as close contact.

\section{Authors' response to our comment \#5.}

The authors said: "We do not conflate the two [close contact transmission and large droplet transmission], hence the need for separate reviews."

This response appears conceptually flawed. If close contact transmission includes droplet, aerosol 
(short-range airborne), as well as indirect contact (fomite) transmission, surely the scope of these reviews overlaps?

We suggest adopting the clearer definition of mechanisms as surface touch, large droplet spray / deposition, and aerosol inhalation suggested by Y. Li (2021) and adopted by the US Centers for Disease Control and Prevention (CDC 2021).

\section{Authors' response to our comment \#6.}

The authors said: "The WHO guidance has not changed."

This response overlooks the fact that we did not say that WHO guidance had changed. We said that the WHO had stated that airborne transmission can occur. To verify our original claim, on 30 April 2021, the WHO updated its 'Covid-19 - how is it transmitted?' page (World Health Organization 2021) with the following statement:

"Current evidence suggests that the virus spreads mainly between people who are in close contact with each other, typically within 1 metre (short-range). A person can be infected when aerosols or droplets containing the virus are inhaled or come directly into contact with the eyes, nose, or mouth. The virus can also spread in poorly ventilated and/or crowded indoor settings, where people tend to spend longer periods of time. This is because aerosols remain suspended in the air or travel farther than 1 metre (longrange)." (our emphasis).

The authors' response does not address our main point here, which was that the authors' statement. "Current evidence from epidemiologic and virologic studies suggest SARS-CoV-2 is primarily transmitted via respiratory droplets and direct and indirect contact", is now outdated, outmoded and frankly, dangerous. In our original comment, we set out several peer-reviewed papers (which are already highly-cited) summarising the evidence base on airborne transmission. We ask, again, that the authors engage with them.

The authors declare "no conflicts of interest". Yet one of the authors, Professor Conly, is the chair of the WHO group that produces the guidelines that have not changed. We question the mechanisms of scientific governance within the WHO which allow the chair of a guideline committee whose views are out of step with mainstream scientific opinion to a) co-author a review "commissioned and paid for by the WHO" which informs the decision-making of that committee, b) present that review as dispassionate by not declaring his WHO position, and c) ignore the WHO's own published advice on mode of transmission.

\section{Authors' response to our comment \#7.}

The authors said: "In our reviews we present and synthesize the original authors' findings and have not ruled out any form of airborne transmission."

We respectfully suggest that "not ruling out" airborne transmission while failing to engage with a strong, consistent and growing evidence base (such as the major indoor / outdoor difference in transmission) that supports a major role for this mode of transmission is both scientifically and ethically inadequate at a time when the world urgently needs policies that accurately reflect the mechanism of transmission of this deadly virus. 


\section{Authors' response to our comment \#8.}

The authors said: "We are working on a method for identifying high quality evidence of transmission causality and testing it by applying it to our future reviews. (See:

https://Www.preprints.org/manuscript/202104.0633/v1)."

Once again our original comment has not been addressed, namely the fact that positive viral culture from clinical specimens was automatically associated with close contact transmission, but not considered for airborne transmission.

For a team that wants to identify high-quality evidence, it would appear that sufficient strength in all the major disciplines involved would be a must. For a disease that a major part of the scientific community thinks is dominantly airborne, it would appear critical to include as part of your team experts on aerosols and airborne transmission. We remain surprised and shocked that the review team includes nobody with expertise in airborne transmission of disease, and we question the WHO's decision to award a contract to a team which lacked this crucial expertise.

\section{Authors' response to our comment \#9.}

The authors said: "We are happy to expand on the methods used to rate the reporting quality of the included studies."

We respectfully suggest that an adequate response to our comment would include such an expansion.

\section{Authors' response to our comment \#10.}

The authors made a number of points here.

Firstly, the authors said: "PCR serves as a trivially simple and fast tool for detecting pathogens, but any virologist would tell you that the link between a nucleic acid signal and an infectious unit are complex and difficult to establish. There's lots of nucleic acid, some small fraction is packaged, and some yet smaller fraction of packaged viruses is infectious. Some aerosol publications assume that 1 nucleic acid signal = 1 infectious particle. This is wrong, in fact for SARS-CoV-2 the ratio is more typically >100,000 if one carefully calibrates the Ct values with internal standards."

In response, and drawing on our collective expertise in virology, we respectfully point out that:

- PCR amplifies viral RNA, each cycle doubling the target RNA (think of rice grains on a chess board), so that after 30 and 40 cycles ( 2 to the 30th and 40th power), the number of copies of the target RNA is $\sim 1$ billion and $\sim 1$ trillion copies. So the ratio should be 1 viable virus to many, many RNA copies. Not the other way around.

- We have not seen any serious, widely accepted claim for 1 viable virus to 1 PCR amplified RNA copy before, for any virus. To give one example, using digital PCR in a study coauthored by one of us in relation to influenza vaccine virus (Kalliomäki et al, page 60), we found that 1 FFU was equal to about 94 RNA copies.

Secondly, the authors said the following: "The authors state that culturing viruses can be tricky. SARS- 
CoV-2 forms large distinctive plaques on a variety a cell lines, as long as they express the ACE2 receptor. Many different cell types have been tested, including cells designed to enhance the growth (e.g. TMPRSS2), all yield decent titers in the $10^{\wedge} 6$ to $10^{\wedge}$ PFU/mL range. Similar titers are retrieved from the most infectious of patient specimens. Yes one needs BSL3 containment, but nowadays if one is looking for infectious SARS-CoV-2, there are no reasons not to grow it."

In response, and drawing on our relevant interdisciplinary expertise, we respectfully point out that this comment needs to be contextualised in that air-sampling substantially disrupts and inactivates lipid-enveloped viruses. We know there is a massive loss of viable virus with such viruses in air samples - as we showed here in our earlier paper on influenza (Brown et al. 2015). That study also showed that RNA copies amplified by PCR also vastly exceed the viable virus count supporting the earlier point made above. But this also means that absence of detected viable virus does not mean that aerosol transmission cannot happen, and the degree to which RNA detection alone can indicate this potential will depend on the context and the availability of other related epidemiological data for that event.

In summary: in aerosol samples, the amount of virions in samples is expected to be low and this will translate into difficulties in culturing viruses, since isolation in cell culture is inherently less sensitive than molecular detection. This is compounded by the fact, well known to virologists, that aerosol collection techniques damage virions and diminish infectivity, so recovery of any infectious SARS-CoV-2 in culture would be highly significant (and this has been done by some groups already) and also almost certainly an underestimate. Low concentration of airborne pathogens is to be expected in aerosol transmission, for example in their studies demonstrating TB transmission from tuberculosis wards Riley et al. (Sultan et al. 1960, Riley et al. 1962) found a concentration of about 1 bacillus per 10000 cubic feet. Aerosol transmission can nonetheless occur because of the very large volume of air inhaled by humans.

In addition, the authors said: "The interesting thing is that when one calculates the titer of virus in culture (TCID50) and measure the minimal infectious dose (MID) in the highly susceptible hamster model, the numbers are surprisingly congruent. The conclusion is that one plaque-forming unit in culture is one hamster infectious unit as well. If there's something wrong with using cell cultures to detect virus and measure titer, one would have to posit that the same exact problem characterizes the hamster model."

In response, we suggest that the authors appear to be missing the point. We are talking about whether or not the isolation of live virus from air samples is a reliable indicator of the aerosol spread of the virus between people - and the degree to which RNA detection can indicate this. We are saying that the lack of such viable virus detection in air-sampling studies does not exclude this possible route of transmission because:

- The air-sampling may be occurring too distant from the SARS-COV-2 sources (in both time and space) with too much dilution and physical shear stress disruption to detect sufficient live virus that is reliably detectable by virus culture;

- We are mostly referring to the context of short-range aerosol transmission in the context of this review - so we should be air-sampling from within $1 \mathrm{~m}$ of the mouth and nose of infected cases - and most environmental sampling studies do not do this; 
- It therefore follows that the negative virus viability with positive RNA findings in these environmental air-sampling studies does not exclude short-range aerosol transmission between people;

- And as air-sampling disrupts the vast majority of lipid-enveloped viruses during the quite violent sampling process - the lack of virus viability in these air-samples is not surprising;

If viable virus is present and detectable by conventional cell culture (as has been reported by some teams already) - in this context - this will also likely underestimate the number of viable viruses initially exhaled by an infectious source.

The comment on the virus viability in the hamster model needs to be better discussed. Infectious dose is not the same by different routes of transmission for many agents e.g. Influenza (Little et al. 1979, Couch et al. 1971, Alford et al. 1966). We note incidentally that aerosol transmission to hamsters and ferrets has been achieved in the laboratory (Port et al 2020, Kutter et al. 2021, Sia et al. 2020). We also note that any animal inoculation experiments (similar to the cell culture methods) attempted with air-sampled virus will also suffer from the above upstream air-sampling virus disruption problems.

So again, any absence of virus infection/viability seen in such animal models does not necessarily exclude airborne transmission of the virus.

Finally, on the authors' comment that PCR is "trivial", we suggest that this comment indicates that none of the authors are practicing clinical virologists working in hospital diagnostician labs otherwise they will be aware of the huge improvements to viral detection and management of viral infections that PCR has contributed to modern clinical medicine. It has been appreciated for many decades that laboratory diagnosis of viral infections by isolation in cell culture was very insensitive and relying solely on this technique was previously causing a lot of false negatives. Already in the 70s techniques of detection of viruses in clinical samples, for example respiratory viruses, by direct fluorescence microscopy was brought to bear to palliate the insensitivity of isolation in cell culture, and globally over the last 20 years, we have replaced most viral culture assays with PCR, on the basis this is a more sensitive method of detection, with less operator subjectivity. This includes routine virus PCR testing (without viral culture confirmation of viability) on:

- CSF (cerebrospinal fluid) - for HSV-1/2, VZV, EV, PeV, CMV, HIV, AdV, JCV, HHV6/7, etc. to diagnose, monitor and treat viral encephalitis - viruses do not culture easily from CSF and some of these viruses (HIV, JCV, HHV6/7) are difficult to grow in conventional cell culture.

- Blood for monitoring AdV, BKV, CMV and EBV viral loads (using quantitative PCR) checking for viral infection/reactivation post-organ transplant - these viruses are not reliably cultured or quantified from blood - as blood has many inhibitors of viral culture.

- Genital swabs for genital herpes infection (mostly from HSV 1-2) - where viral culture has been found to be less sensitive than PCR in multiple studies - so this risks missing earlier 
lesions that could be treated earlier to reduce transmission to others, as well as for improved clinical outcomes for patients.

- Respiratory viruses - PCR has again proven much more sensitive - to detect RSV, parainfluenza, influenza, AdV, hMPV and multiple other seasonal respiratory viruses - not all of which grow easily in viral culture or are detected at low level by IF - again naso/oropharyngeal swabs can pick up inhibitors to viral culture.

In sum, we remain concerned that these authors may lack key expertise in their team and that as a result, their interpretation of the evidence and the conclusions they draw are potentially misleading and dangerous.

Prof. Trisha Greenhalgh, Dept. of Primary Care Health Sciences, Medical Sciences Div., Univ. of Oxford, UK

Dr. Julian W Tang, Respiratory Sciences, University of Leicester, Leicester, UK

Dr Hidekazu Nishimura, Virus Research Center, Clinical Research Division, Sendai Medical Center, National Hospital Organization, Sendai, Japan

Prof. Jose L. Jimenez, Dept. of Chemistry \& CIRES, Univ. of Colorado, Boulder, CO, USA

Prof. Stephanie J. Dancer, Dept. of Microbiology, Hairmyres Hospital, Glasgow, and Edinburgh Napier University, UK

Prof. Giorgio Buonanno, Dept. of Civil and Mechanical Engineering, University of CAssino and Southern Lazio, Italy

Prof. Lidia Morawska, ILAQH,Queensland University of Technology; Vice-Chancellor Fellow, Global Centre for Clean Air Research (GCARE), University of Surrey, UK

Prof. William Bahnfleth, Dept. of Architectural Engineering, The Pennsylvania State University, University Park, PA, USA

\section{References}

Alford, R. H., J. A. Kasel, P. J. Gerone, and V. Knight. 1966. “Human Influenza Resulting from Aerosol Inhalation." Proceedings of the Society for Experimental Biology and Medicine. Society for Experimental Biology and Medicine 122 (3): 800-804.

Brown, J. R., J. W. Tang, L. Pankhurst, N. Klein, V. Gant, K. M. Lai, J. McCauley, and J. Breuer. 2015. “ Influenza Virus Survival in Aerosols and Estimates of Viable Virus Loss Resulting from Aerosolization and Air-Sampling." The Journal of Hospital Infection 91 (3): 278-81.

Centers for Disease Control and Prevention (2021). Scientific Brief: SARS-CoV-2 Transmission (updated May 7th 2021). 
Couch, R. B., R. G. Douglas Jr, D. S. Fedson, and J. A. Kasel. 1971. “Correlated Studies of a Recombinant Influenza-Virus Vaccine. 3. Protection against Experimental Influenza in Man." The Journal of Infectious Diseases 124 (5): 473-80.

Couch, R. B., J. A. Kasel, J. L. Gerin, J. L. Schulman, and E. D. Kilbourne. 1974. “Induction of Partial Immunity to Influenza by a Neuraminidase-Specific Vaccine." The Journal of Infectious Diseases 129 (4): 411-20.

Kalliomäki, P., H. Koskela, M. Waris, and J. W. Tang. n.d. "Assessing the Risk to Healthcare Workers of Hospital-Acquired Infection from Patients Infected with Aerosol-Transmissible Pathogens."

Kutter, J.S., de Meulder, D., Bestebroer, T.M. et al. SARS-CoV and SARS-CoV-2 are transmitted through the air between ferrets over more than one meter distance. Nat Commun 12, 1653 (2021).

Li Y. 2021. Basic routes of transmission of respiratory pathogens-A new proposal for transmission categorization based on respiratory spray, inhalation, and touch. Indoor Air, 31, 3-6.

Little, J. W., R. G. Douglas Jr, W. J. Hall, and F. K. Roth. 1979. “Attenuated Influenza Produced by Experimental Intranasal Inoculation." Journal of Medical Virology 3 (3): 177-88.

Port Julia R, Claude Kwe Yinda, Irene

Offei Owusu, Myndi Holbrook, Robert Fischer, Trenton Bushmaker, Victoria A. Avanzato, Jonathan E. Schulz, Neeltje van Doremalen, Chad S. Clancy, Vincent J. Munster SARS-CoV-2 disease severity and transmission efficiency is increased for airborne but not fomite exposure in Syrian hamsters.

bioRxiv 2020.12.28.424565

Prather, Kimberly A., Linsey C. Marr, Robert T. Schooley, Melissa A. McDiarmid, Mary E. Wilson, and Donald K. Milton. 2020. “Airborne Transmission of SARS-CoV-2." Science 370 (6514): 303-4.

Randall, Katherine, E. Thomas Ewing, Linsey Marr, Jose Jimenez, and L. Bourouiba. 2021. “How Did We Get Here: What Are Droplets and Aerosols and How Far Do They Go? A Historical Perspective on the Transmission of Respiratory Infectious Diseases."

Riley, R. L., C. C. Mills, F. O'grady, L. U. Sultan, F. Wittstadt, and D. N. Shivpuri. 1962. “Infectiousness of Air from a Tuberculosis Ward. Ultraviolet Irradiation of Infected Air: Comparative Infectiousness of Different Patients." The American Review of Respiratory Disease 85 (April): 511-25.

Samet, Jonathan M., Kimberly Prather, Georges Benjamin, Seema Lakdawala, John-Martin Lowe, Arthur Reingold, John Volckens, and Linsey Marr. 2021. "Airborne Transmission of SARS-CoV-2: What We Know." Clinical Infectious Diseases: An Official Publication of the Infectious Diseases Society of America, January.

Sia, S.F., Yan, L.M., Chin, A.W., Fung, K., Choy, K.T., Wong, A.Y., Kaewpreedee, P., Perera, R.A., Poon, L.L., Nicholls, J.M. and Peiris, M., 2020. Pathogenesis and transmission of SARS-CoV-2 in golden 
hamsters. Nature, 583(7818), pp.834-838.

Sultan, L., W. Nyka, C. Mills, F. O'grady, W. Wells, and R. L. Riley. 1960. “Tuberculosis Disseminators. A Study of the Variability of Aerial Infectivity of Tuberculous Patients." The American Review of Respiratory Disease 82 (September): 358-69.

Wells, W. F. 1934. “ON AIR-BORNE INFECTION*: STUDY II. DROPLETS AND DROPLET NUCLEI.” American Journal of Epidemiology 20 (3): 611-18.

World Health Organization. 2020. "Transmission of SARS-CoV-2: Implications for Infection Prevention Precautions," July.

—_- 2021. “Coronavirus Disease (COVID-19): How Is It Transmitted?," April.

Xie, X., Y. Li, A. T. Y. Chwang, P. L. Ho, and W. H. Seto. 2007. “How Far Droplets Can Move in Indoor Environments--Revisiting the Wells Evaporation-Falling Curve." Indoor Air 17 (3): 211-25.

Competing Interests: No competing interests were disclosed.

Author Response 11 Jun 2021

IGHO ONAKPOYA, University of Oxford, Oxford, UK

We would like to respond to the public comments by Prof. Jimenez and colleagues regarding our systematic review assessing the role of close contact in the transmission of SARS-CoV-2. We have itemized our responses respectively in line with each of the comments.

Response to comment \#1. Thank you. We have found conflicting definitions of particle size and the issue of short vs long range transmission is complex and requires a common set of agreed upon definitions and more study.

Response to comment \#2. As we make clear in the text, we draw no definitive conclusions on this issue. We do not "believe" anything without solid evidence.

Response to comment \#3. We find this comment useful and propose close proximity (without physical contact) and close contact.

Response to comment \#4. Once an agreed upon set of revised definitions is achieved, we and others would use them in any reviews of the evidence.

Response to comment \#5. We do not conflate the two, hence the need for separate reviews.

Response to comment \#6. The WHO guidance has not changed.

Response to comment \#7. In our reviews we present and synthesize the original authors' findings 
and have not ruled out any form of airborne transmission.

Response to comment \#8. We are working on a method for identifying high quality evidence of transmission causality and testing it by applying it to our future reviews. (See:

https://www.preprints.org/manuscript/202104.0633/v1)

Response to comment \#9. We are happy to expand on the methods used to rate the reporting quality of the included studies.

Response to comment \#10. PCR serves as a trivially simple and fast tool for detecting pathogens, but any virologist would tell you that the link between a nucleic acid signal and an infectious unit are complex and difficult to establish. There's lots of nucleic acid, some small fraction is packaged, and some yet smaller fraction of packaged viruses is infectious. Some aerosol publications assume that 1 nucleic acid signal $=1$ infectious particle. This is wrong, in fact for SARSCoV-2 the ratio is more typically $>100,000$ if one carefully calibrates the Ct values with internal standards Moreover, numerous studies have shown that while the PCR signal can persist for long periods of time, infectious virus are detected over a very narrow window of time, typically 7-10 days in otherwise immune competent individuals. (see Jefferson et al.) Ct values $<25$ are required to detect virus (from which one can calculate things like the RNA/PFU ratio).

The authors state that culturing viruses can be tricky. SARS-CoV-2 forms large distinctive plaques on a variety a cell lines, as long as they express the ACE2 receptor. Many different cell types have been tested, including cells designed to enhance the growth (e.g. TMPRSS2), all yield decent titers in the $10^{\wedge} 6$ to $10^{\wedge} \mathrm{PFU} / \mathrm{mL}$ range. Similar titers are retrieved from the most infectious of patient specimens. Yes one needs BSL3 containment, but nowadays if one is looking for infectious SARSCoV-2, there are no reasons not to grow it.

The interesting thing is that when one calculates the titer of virus in culture (TCID50) and measure the minimal infectious dose (MID) in the highly susceptible hamster model, the numbers are surprisingly congruent. The conclusion is that one plaque-forming unit in culture is one hamster infectious unit as well. If there's something wrong with using cell cultures to detect virus and measure titer, one would have to posit that the same exact problem characterizes the hamster model.

Dr. Igho J. Onakpoya, University of Oxford, Centre for Evidence-Based Medicine, Nuffield Department of Primary Care Health Sciences, Oxford UK

Prof. Carl J. Heneghan, University of Oxford, Centre for Evidence-Based Medicine, Nuffield Department of Primary Care Health Sciences, Oxford UK

Dr. Elizabeth A. Spencer, University of Oxford, Centre for Evidence-Based Medicine, Nuffield Department of Primary Care Health Sciences, Oxford UK

Jon Brassey, Trip Database Ltd, Little Maristowe, Glasllwch Lane, Newport, UK

Dr. Annette Plüddemann, University of Oxford, Centre for Evidence-Based Medicine, Nuffield 


\section{Department of Primary Care Health Sciences, Oxford UK}

Prof. David H. Evans, Department of Medical Microbiology \& Immunology at Li Ka Shing Institute of Virology, University of Alberta, Canada

Prof. John M. Conly, University of Calgary and Alberta Health Services, Calgary, Canada.

Dr. Tom Jefferson, University of Oxford, Centre for Evidence-Based Medicine, Nuffield Department of Primary Care Health Sciences, Oxford UK

Competing Interests: N/A

Reader Comment 31 May 2021

Jose-Luis Jimenez, University of Colorado-Boulder, Boulder, Colorado, USA

\section{Public comment on Onakpoya et al. Review on Close Contact Transmission}

We would like to offer some comments on the systematic review of Onakpoya et al. on close contact transmission of COVID-19.

First, we commend the authors for using the F1000 platform that allows posting of public comments from any scientist during peer review. This allows interested scientists to bring up issues and evidence that may not have been considered by the authors, and thus more crosspollination than would be possible with traditional anonymous closed peer-review.

However, there are some major problems with the choice of the topic as "close contact transmission," and the framing and description of the topic by Onakpoya et al.

1) The other reviews by this group concern airborne, orofecal, and fomite transmission, all of which are actual transmission mechanisms. However, "close contact" is a measurement of distance, not a mechanism of transmission.

The authors acknowledge this when they state, "The mechanistic pathway for transmission in close contact settings is unclear", and also mention, "there is lack of clarity about how research should be conducted in the setting of transmission with close contact which may include transmission via any one of or the combination of respiratory droplets, direct contact, or indirect contact."

However, short-range airborne transmission is never mentioned, even though recent results indicate that it is the dominant transmission mechanism between talking individuals, and is also important when coughing (Chen et al. 2020). Talking in close proximity is important for presymptomatic, asymptomatic, and mildly-symptomatic (PAMS) individuals, which are important contributors to transmission in this pandemic (Johansson et al. 2021; Jones et al. 2021). For clarity, short-range airborne transmission is the inhalation of respiratory aerosols while in close proximity (Chen et al. 2020; Li 2021; Milton 2020). Those are the same respiratory aerosols that can cause 
infection at larger distances when dispersed in shared room air, a mechanism that can explain most superspreading events (Miller et al. 2021; Greenhalgh et al. 2021a).

Aerosols are much more concentrated in exhaled breath from an infectious person than in room air. Thus, for airborne diseases that can infect through shared room air despite large dilution, they would naturally be much more infectious in close proximity (Tang et al. 2021a; Tang et al. 2021b).

In the revision of this paper, the authors must acknowledge the recent scientific literature, including the likelihood that "close contact" transmission is actually dominated by short-range airborne transmission.

2) Equally important, are the authors preparing a systematic review on large droplet transmission of SARS-CoV-2? That is the mechanism that WHO and some of the co-authors of this systematic review have upheld as dominant throughout much of the pandemic (Conly et al. 2020; World Health Organization 2020). Thus, it would behove the authors to synthesize the presumably strong evidence that large droplets are actually important for transmission. If they are indeed so important, it should not be hard to compile strong and abundant evidence?

A recent review of the literature by Prof. Yuguo Li (a current member of the WHO IPC R\&D committee (World Health Organization 2021b)) and colleagues concludes that, "reviewing the literature of large droplet transmission, one can find no direct evidence for large droplets as the route of transmission of any disease" (Chen et al. 2020). If Onakpoya et al. disagree, and believe that droplet transmission has been directly demonstrated for any disease, we would appreciate the addition of key references in their response.

As we discuss below, equating ease of infection in close proximity with droplet transmission is a major conceptual error that has prevailed for over a century.

3) The term "close contact" is also ambiguous, since the word "contact" is used, implying direct physical contact. However, any situation in close proximity may be described as "close contact," whether physical contact actually occurred or not. We would like to suggest "close proximity" as a clearer term for future use.

4) A pervasive error in the field of disease transmission has been equating "close contact transmission" with large droplet, direct contact, and fomite transmission only, and excluding airborne transmission. It is important to correct this error, and to recognize that transmission in close proximity is likely dominated by short-range airborne transmission, via aerosol inhalation (Chen et al. 2020).

The error conflating ease of infection in close proximity with droplet transmission dates from the work of Dr. Charles Chapin in 1910. Dr. Chapin conceptualized contact infection in his seminal book, "The sources and modes of infection" (Chapin 1912). He realized that ease of infection in close proximity could be explained by large droplets that fell to the ground close to the infected person, a mechanism that he referred to as "spray-borne" transmission, or by inhalation of smaller particles (now commonly referred to as aerosols) through airborne transmission. Despite lacking 
evidence, he expressed his opinion that spray-borne infection was dominant in close proximity and airborne infection was unlikely to be important: "It will be a great relief to most persons to be freed from the specter of infected air, a specter which has pursued the race from the time of Hippocrates." This was due partly to his desire to win acceptance for the concept of contact infection, which he considered the most important mode of infection, since (in his words), "it is impossible, as I know from experience, to teach people to avoid contact infection while they are firmly convinced that the air is the chief vehicle of infection" (Chapin 1912).

This conflation of ease of infection in close proximity with large droplets became widely accepted, despite the (continuing) lack of evidence to support it (Chen et al. 2020). It has become a dogma in this field for over a century (Randall et al. 2021). Supporting evidence has often been attributed to the work of Carl Flugge, and large droplets are referred to as "Flugge's droplets." Although only visible spray-borne droplets had been considered for disease transmission by some earlier researchers (Cornet 1889), Flugge and coworkers in the 1890s used the term "droplets" to refer to both droplets and aerosols. In their experiments, they often waited up to 5 hours to allow settling of smaller aerosols onto their collection plates (Randall et al. 2021; Chapin 1912).

5) The same error of conflating ease of infection in close proximity with large droplet transmission was used to designate measles as a droplet/fomite disease until about 1985 (Bloch et al. 1985). It is now clear that much, or all, of the close proximity transmission of measles that had been attributed to droplets is indeed short-range airborne transmission. There is no fundamental reason why this error cannot apply to other diseases. Tuberculosis is also most easily acquired in close proximity, yet it can only be transmitted through the airborne mechanism (Sepkowitz 1996). Ease of transmission in close proximity is also observed for chickenpox, another well-known airborne pathogen (CDC 2021a).

6) The statement by Onakpoya et al. that, "Current evidence from epidemiologic and virologic studies suggest SARS-CoV-2 is primarily transmitted via respiratory droplets and direct and indirect contact", is now outdated, outmoded and frankly, dangerous.

Both the WHO (World Health Organization 2021a) and the US CDC (CDC 2021b) have recently accepted that airborne transmission is an important form of transmission for SARS-CoV-2. This follows overwhelming scientific evidence that has accumulated in support of airborne transmission, which many scientists now consider the predominant mode of transmission of SARSCoV-2 (Greenhalgh et al. 2021a). We now have a broader understanding of the long-standing errors and misinterpretations about airborne transmission that led to it being underappreciated for many diseases (Tang et al. 2021a; Tang et al. 2021 b; Randall et al. 2021; Chen et al. 2020; Greenhalgh et al. 2021a; Greenhalgh, et al. 2021b).

7) The authors note that, "Enclosed environments and social gatherings appear to increase the likelihood of close contact transmission." This can be explained by short-range airborne transmission, due to greatly reduced dispersion of aerosols in inadequately ventilated indoor environments. Typical air speeds outdoors are at least 10 times larger than indoors. However, increased indoor transmission cannot be easily explained by large droplets since gravity is the same indoors and outdoors. If the authors have a mechanistic explanation of how enclosed environments would increase transmission, in the absence of airborne transmission, it 
should be presented in the review.

8) Onakpoya et al. state, in support of close contact transmission, "Our review [...] shows evidence demonstrating positive culture of virus as well as genomic evidence of close contact transmission. This differs from the findings from our reviews of fomite, orofecal and airborne transmission that failed to show evidence of either positive culture or genomic sequences demonstrating SARS-CoV-2 transmission."

We thus expected to find studies where the virus had been cultivated from samples of large droplets or samples taken from e.g. hands. However, the two cited studies with positive viral culture performed the culture analyses of nasal swabs (Ladhani et al. 2020) and of, "throat swab and bilateral nasopharyngeal or deep nasal specimens" (Speake et al. 2020). It is a logical error to associate the presence of cultivable virus from clinical specimens taken from an infected person with 'close contact transmission' (especially as narrowly defined here, excluding shortrange airborne transmission) and not with other forms of transmission. Similarly one may find cultivable tuberculosis, measles, and chickenpox pathogens from clinical specimens, but that does not mean that transmission in close proximity is explained by large droplets or direct or indirect contact. The most likely (measles, chickenpox) or only (tuberculosis) mechanism of transmission of those diseases in close proximity will be short-range airborne transmission. Therefore the erroneous association of positive cultures of nasal swabs as supporting a narrow definition of close contact transmission needs to be removed.

9) We question the use of the "modified" QUADAS 2 risk of bias tool as the sole arbiter of quality in the primary studies analyzed for this review. QUADAS 2 was designed for diagnostic accuracy studies in which a new test is compared with an existing gold standard in a representative sample of participants (Whiting et al. 2011). It is essentially an epidemiological tool whose purpose is to ensure that the sample was representative and the 'gold standard' comparator is a legitimate gold standard for the disease or risk state in question. The 'biases' it identifies relate to such things as sampling of participants (e.g. do the patients in the study accurately represent the range of patients with the target condition?), sample size, completeness of follow-up and so on. QUADAS 2 was not designed to assess essential aspects of quality in laboratory studies. Extraordinarily, the authors do not even state how they modified the QUADAS 2 tool and they certainly make no attempt to justify or defend this modification. We question, therefore, whether the process adopted for assigning studies to "low", "medium" or "high" quality is valid. At a minimum, the fitness of purpose as well as the modifications of the QUADAS2 tool need to be described in detail and justified in the revised version of this paper.

10) Using in vitro viral culture as the gold standard to define the potential for virus transmissibility between humans is not a sensitive approach. Indeed, many clinical viral infections are treated on the basis of PCR results alone. Viral culture is not a sensitive method to detect viruses - it has never been (van Elden et al. 2002). It is one of the 'catch-all' methods used in virology to identify the presence and amplify new viruses - like electron-microscopy - both of which need hundreds to thousands of viruses to enable detection and identification. For cell culture also, one needs to identify the optimal cell-line and this differs between viruses. The authors are already off to a bad start when they are proposing viral culture as the 'gold standard' of detection for any virus especially respiratory viruses when there are multiple cell-line options for culturing them - as they 
then need to define which ones should be used as the 'gold standard' for each virus and there is not always just one option for this.

Virologists know that just one virus of any species will not necessarily culture successfully in any cell culture system - you need a minimum viral load to be successful in viral culture, which can vary between viruses and cell-lines. So if it cultures, then that is a positive result (assuming no lab contamination has occurred), but if not, it does not mean that there is no infective virus present. Different viral species and variants have different tropisms and therefore different degrees of success in growing in different cell-lines under different conditions.

Assigning 'gold standard' criteria for viral culture as proof of viral transmission is neither sensitive (it can give false negative results), nor reliable (it may not be always reproducible), nor robust (too many factors, including the way that the sample was collected and stored, can significantly perturb this process). Suggesting that serial culture is needed in a 'gold standard' deepens the problems even more.

Prof. Jose L. Jimenez, Dept. of Chemistry \& CIRES, Univ. of Colorado, Boulder, CO, USA

Prof. Stephanie J. Dancer, Dept. of Microbiology, Hairmyres Hospital, Glasgow, and Edinburgh Napier University, UK

Prof. Trisha Greenhalgh, Dept. of Primary Care Health Sciences, Medical Sciences Div., Univ. of Oxford

Prof. Linsey C. Marr, Dept. of Civil and Environmental Engineering, Virginia Tech, Blacksburg, VA, USA

Dr. Julian W Tang, Respiratory Sciences, University of Leicester, Leicester, UK

Prof. David Fisman, Dalla Lana School of Public Health, University of Toronto, Toronto, ON, Canada

Prof. Giorgio Buonanno, Dept. of Civil and Mechanical Engineering, University of Cassino and Southern Lazio, Cassino, Italy

\section{References}

Bloch AB., Orenstein WA., Ewing WM., Spain WH., Mallison GF., Herrmann KL., and Hinman AR. 1985. "Measles Outbreak in a Pediatric Practice: Airborne Transmission in an Office Setting." Pediatrics 75 (4): 676-83.

CDC. 2021a. "Chickenpox (Varicella) Transmission.” URL:

https://www.cdc.gov/chickenpox/about/transmission.html [Accessed April 28, 2021].

CDC. 2021b. "Scientific Brief: SARS-CoV-2 Transmission." URL:

https://www.cdc.gov/coronavirus/2019-ncov/science/science-briefs/sars-cov-2-transmission.html. [Updated May 7, 2021]. 
Chapin, CV. 1912. The Sources and Modes of Infection. New York, J. Wiley \& sons. URL: https://lccn.loc.gov/12021189

Chen W., Zhang N., Wei J., Yen H., Li Y. 2020. "Short-Range Airborne Route Dominates Exposure of Respiratory Infection during Close Contact." Building and Environment 176 (June): 106859.

Conly J., Seto WH., Pittet D., Holmes A., Chu M., Hunter PR., WHO Infection Prevention and Control Research and Development Expert Group for COVID-19. 2020. “Use of Medical Face Masks versus Particulate Respirators as a Component of Personal Protective Equipment for Health Care Workers in the Context of the COVID-19 Pandemic." Antimicrobial Resistance and Infection Control 9 (1): 126.

Cornet, Georg. 1889. Über Tuberculose: die Verbreitung der Tuberkelbacillen ausserhalb des Körpers (German Edition). Hansebooks.

van Elden LJR., van Kraaij MGJ., Nijhuis M., Hendriksen KAW., Dekker AW., Rozenberg-Arska M., and van Loon AM. 2002. “Polymerase Chain Reaction Is More Sensitive than Viral Culture and Antigen Testing for the Detection of Respiratory Viruses in Adults with Hematological Cancer and Pneumonia." Clinical Infectious Diseases: An Official Publication of the Infectious Diseases Society of America 34 (2): 177-83.

Greenhalgh T., Jimenez JL., Prather KA., Tufekci Z., Fisman D., Schooley R. 2021 a. “Ten Scientific Reasons in Support of Airborne Transmission of SARS-CoV-2." The Lancet 397 (10285): 1603-1605.

Greenhalgh T., Ozbilgin M., Contandriopoulos D. 2021 Orthodoxy, illusio, and playing the scientific game: a Bourdieusian analysis of infection control science in the COVID-19 pandemic [version 1; peer review: awaiting peer review]. Wellcome Open Res (6):126

Johansson MA., Quandelacy TM., Kada S., Prasad PV., Steele M., Brooks JT., Slayton RB., Biggerstaff M., Butler JC. 2021. "SARS-CoV-2 Transmission From People Without COVID-19 Symptoms." JAMA Network Open 4 (1): e2035057.

Jones TC., Biele G., Mühlemann B., Veith T., Schneider J., Beheim-Schwarzbach J., Bleicker T., et al. 2021. “Estimating Infectiousness throughout SARS-CoV-2 Infection Course." Science, (May): eabi5273.

Ladhani SN., Chow JY., Janarthanan R., Fok J., Crawley-Boevey E., Vusirikala A., Fernandez E., et al. 2020. "Investigation of SARS-CoV-2 Outbreaks in Six Care Homes in London, April 2020." EClinicalMedicine 26 (September): 100533.

Yuguo L. 2021. “Basic Routes of Transmission of Respiratory Pathogens-A New Proposal for Transmission Categorization Based on Respiratory Spray, Inhalation, and Touch." Indoor Air 31 (1): 3-6.

Miller SL., Nazaroff WM., Jimenez JL., Boerstra A., Buonanno G., Dancer SJ., Kurnitski J., Marr LC., Morawska L., Noakes C. 2021. “Transmission of SARS-CoV-2 by Inhalation of Respiratory Aerosol in 
the Skagit Valley Chorale Superspreading Event." Indoor Air 31 (2): 314-23.

Milton DK. 2020. "A Rosetta Stone for Understanding Infectious Drops and Aerosols." Journal of the Pediatric Infectious Diseases Society 9 (4): 413-15.

Randall, KE., Ewing T., Marr L., Jimenez J., Bourouiba L. 2021. “How Did We Get Here: What Are Droplets and Aerosols and How Far Do They Go? A Historical Perspective on the Transmission of Respiratory Infectious Diseases." URL: https://papers.ssrn.com/abstract=3829873.

Sepkowitz, KA. 1996. “How Contagious Is Tuberculosis?" Clinical Infectious Diseases: An Official Publication of the Infectious Diseases Society of America 23 (5): 954-62.

Speake H., Phillips A., Chong T., Sikazwe C., Levy A., Lang J., Scalley B., et al. 2020. “Flight-Associated Transmission of Severe Acute Respiratory Syndrome Coronavirus 2 Corroborated by WholeGenome Sequencing." Emerging Infectious Diseases 26 (12): 2872-80.

Tang JW., Marr LC., Milton DK. 2021b. "Aerosols Should Not Be Defined by Distance Travelled." The Journal of Hospital Infection (May).

Tang JW., Bahnfleth WP., Bluyssen PM., Buonanno G., Jimenez JL., Kurnitski J., Li Y., et al. 2021a. “ Dismantling Myths on the Airborne Transmission of Severe Acute Respiratory Syndrome Coronavirus-2 (SARS-CoV-2)." The Journal of Hospital Infection 110 (April): 89-96.

Whiting PF., Rutjes AWS., Westwood ME., Mallett S., Deeks JJ., Reitsma JB., Leeflang MMG., Sterne JAC., Bossuyt PMM., QUADAS-2 Group. 2011. “QUADAS-2: A Revised Tool for the Quality Assessment of Diagnostic Accuracy Studies." Annals of Internal Medicine 155 (8): 529-36.

World Health Organization (WHO Headquarters). 2020. “Transmission of SARS-CoV-2: Implications for Infection Prevention Precautions." World Health Organization. July 9, 2020. URL:

https://www.who.int/news-room/commentaries/detail/transmission-of-sars-cov-2-implications-forinfection-prevention-precautions.

World Health Organization. 2021a. "Coronavirus Disease (COVID-19): How Is It Transmitted?" April 30, 2021. URL: https://www.who.int/news-room/q-a-detail/coronavirus-disease-covid-19-how-is-ittransmitted?

World Health Organization. 2021b. "WHO COVID-19 Infection Prevention and Control (IPC) Pillar Achievements. February 2020 - January 2021." URL: https://www.who.int/publications/m/item/whocovid-19-infection-prevention-and-control-(ipc)-pillar [Accessed May 31, 2021].

Competing Interests: The authors declare no competing interest.

Reader Comment 21 Apr 2021

David Tomlinson, University Hospitals Plymouth NHS Trust, Plymouth, UK 
Dear Dr Onakpoya and team,

Thank you for posting your article 'SARS-CoV-2 and the role of close contact in transmission: a systematic review [version 1; peer review: awaiting peer review]' on the F1000Research site and for inviting comments. I hope you find the following comments to be constructive, free from bias and useful towards improving your manuscript.

\section{Introduction}

'Current evidence from epidemiologic and virologic studies suggest SARS-CoV-2 is primarily transmitted via respiratory droplets and direct and indirect contact ${ }^{2,3.1}$

Ref 2: 'World Health Organization: Modes of transmission of virus causing COVID-19: implications for IPC precaution recommendations. [Accessed 16/01/2021]' links to WHO 'Modes of transmission of virus causing COVID-19: implications for IPC precaution recommendations, Scientific brief, 29 March 2020.' Ref 3: 'World Health Organization: Coronavirus disease 2019 (COVID-19) Situation Report - 73. [Accessed 28 February 2021]' link does not work.

On $5^{\text {th }}$ April 2021 and in updated guidance the CDC stated: "Findings of these studies suggest that the risk of SARS-CoV-2 infection via the fomite transmission route is low, and generally less than 1 in 10,000, which means that each contact with a contaminated surface has less than a 1 in 10,000 chance of causing an infection.". Furthermore, since the latest WHO IPC Scientific Brief, July 2020, much more evidence towards the dominant role for airborne transmission of SARS-CoV-2 has been identified: investigations supporting this proposition include the recovery of live virus from the air, animal transmission studies using models excluding fomite and/or droplet routes, and detailed epidemiological investigations into transmission events possible only via the airborne (short and long-range) route. Greenhalgh T et al. (2021) recently reviewed and described these lines of evidence, concluding: 'There is consistent, strong evidence that SARS-CoV-2 spreads by airborne transmission. Although other routes can contribute, we believe that the airborne route is likely to be dominant.'.

Therefore, I would be grateful if the authors would change this sentence to reflect these most recently available data, please. Thank you.

Box 1: terminology.

Re: 'Close contact: Someone who was within 6 feet of an infected person for a cumulative total of 15 minutes or more over a 24-hour period starting from 2 days before illness onset (or, for asymptomatic patients, 2 days prior to test specimen collection) until the time the patient is isolated;1 The World Health Organization (WHO) additionally includes direct physical contact with a probable or confirmed case, direct care for a patient with probable or confirmed COVID-19 disease without using proper personal protective equipment, and other situations as indicated by local risk assessments.'

The link provided is for a CDC site titled: 'Operational Considerations for Adapting a Contact Tracing Program to Respond to the COVID-19 Pandemic in non-US Settings'. The text used at this CDC site is an almost identical to that used in this present manuscript: 'Close contact is defined by CDC as 
someone who was within 2 meters of an infected person for at least 15 minutes within a 24-hour period starting from 2 days before illness onset (or, for asymptomatic cases 2 days prior to positive specimen collection) until the time the patient is isolated. The World Health Organization (WHO) additionally includes persons with direct physical contact with a probable or confirmed case, direct care for a patient with probable or confirmed COVID-19 disease without using proper personal protective equipment, and other situations as indicated by local risk assessments.'

Given the similarity in wording and the fact that the WHO is mentioned as appropriate by the authors, I would be grateful if the authors could include reference to this being the CDC definition as appropriate, please. Also, omission of the word 'positive' in this sentence is in error and should be corrected please: ' 2 days prior to test specimen collection'. Thank you.

Re: 'Attack rate: The proportion of those who become ill after a specified exposure?'.

This link is blocked. When I typed link 2 web address in directly, I reached a WHO webpage stating: "This page cannot be found. The page or file you are trying to access cannot be found. This is because the web address is incorrect or the file has been moved or deleted. In 2020, we migrated our web content to a new system so some older content may no longer be available online or at the same place."

I would be grateful if the authors would correct this please. Thank you.

Re: 'Secondary attack rate: The probability that infection occurs among susceptible persons within a reasonable incubation period following known contact with an infectious person or an infectious source ${ }^{3}$.

The reference given is a textbook which cannot be readily accessed. Furthermore, the wording 'within a reasonable incubation period' seems insufficiently exact: given that this manuscript is only focused on the assessment of a single pathogen, could the authors provide a more appropriate and reproducible definition of a time interval for SARS-CoV-2 secondary attack rate, please? Thank you.

\section{Methods}

Re: 'published protocol last updated on the 1 December 2020 (Version 3: 1 December 2020, Extended data: Appendix 16)'

This Appendix document ('Web Appendix 1) states: 'For the respective topics we used the following terms:

Airborne: aerosol OR airborne OR airbourne OR inhalation OR air OR droplet Orofecal: orofecal OR oro-fecal OR faecal OR fecal OR stool OR faeces OR feces OR rectal OR rectum OR anal $O R$ anus $O R$ toilet

Fomite: fomite OR surfaces

These terms were also combined with the term transmission.' 
The search terms for the present topic 'close contact' are not listed here. Please could the authors provide a complete list of search terms employed for this present manuscript? Thank you.

Web Appendix 1 states: 'Objectives

Objectives are to provide a rapid summary and evaluation of relevant data on transmission of SARS-CoV2, report important policy implications, and highlight areas of research urgently needed. These transmission areas include airborne, contact and droplet, orofecal, vertical, fomite and other modes such as urine and blood and body fluids.'

The 'transmission area' of 'close contact' is not listed here. Could the authors please explain why? Thank you.

Re: 'We assessed the risk of bias of included primary studies using five domains from the QUADAS-2 criteria ${ }^{7}$; we adapted this tool because the included studies were not primarily designed as diagnostic accuracy studies.'

The QUADAS-2 tool is for 'more transparent rating of bias and applicability of primary diagnostic accuracy studies'. The authors state that they 'adapted this tool' and yet you no description of the adaptation methods is provided. Accordingly, these methods are neither transparent, nor reproducible - a contradiction to the stated intent of the authors who developed the QUADAS-2 tool. Please could the authors explain how this tool was adapted for this present manuscript, in terms allowing an assessment of its methodological validity? Thank you.

Finally, I hope the authors don't mind me providing a more general comment? I have concerns regarding the stated aim towards performing a systematic review of the possible 'role of close contact' in [SARS-CoV-2] transmission. The authors will of course be aware that 'Close contact' is a new classification when considering presently accepted modes of transmission. For example, the terminology below is endorsed by the CDC:

"An infectious agent may be transmitted from its natural reservoir to a susceptible host in different ways. There are different classifications for modes of transmission. Here is one classification:

Direct

- Direct contact

- Droplet spread

Indirect

- Airborne

- Vehicle-borne

- Vector-borne (mechanical or biologic)'

Source: Principles of Epidemiology in Public Health Practice, Third Edition, An Introduction to Applied Epidemiology and Biostatistics. 
Furthermore, the scientific basis for the definition of 'close contact' is without suitable validation, since the stated source for this definition is a document titled: 'Operational Considerations for Adapting a Contact Tracing Program to Respond to the COVID-19 Pandemic in non-US Settings.' Clearly, this 'operational' manuscript does not prove the independent benefit or validity of a new 'close contact' nomenclature towards describing the transmission of an infectious disease.

Therefore, I believe it is important for the authors to explain how and why they came to derive this novel terminology, including a description of its scientific basis with an explanation of how the authors believe it to be a necessary introduction to infectious diseases terminology in addition to the presently accepted and objectively defined modes of transmission. Thank you.

I hope the authors will understand that from my comments above, it is my assertion that the methods employed in this present manuscript are so importantly flawed and/or inadequately described, that neither the results nor conclusions of this present manuscript can be considered to be scientifically valid.

I would once more like to thank the authors for providing the opportunity for comments to be made in response to this present manuscript. I genuinely admire this approach: a research team willing to declare manuscripts open to peer review, and also hopefully open to the possibility that comments received may help towards improving the quality of their final published work.

Competing Interests: I have no conflicts of interest.

The benefits of publishing with F1000Research:

- Your article is published within days, with no editorial bias

- You can publish traditional articles, null/negative results, case reports, data notes and more

- The peer review process is transparent and collaborative

- Your article is indexed in PubMed after passing peer review

- Dedicated customer support at every stage

For pre-submission enquiries, contact research@f1000.com

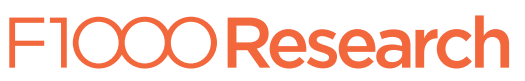

